Linköping Studies in Science and Technology

Dissertations, No. 1936

\title{
Machine Learning-Based Bug Handling in Large-Scale Software Development
}

\author{
Leif Jonsson
}

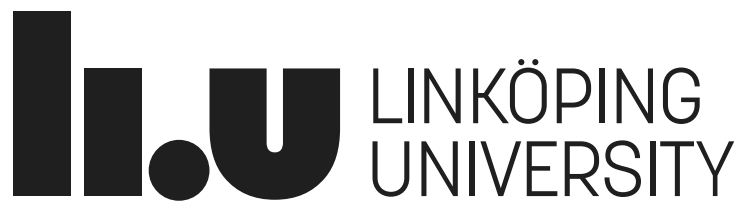

Department of Computer and Information Science SE-581 83 Linköping, Sweden

Linköping 2018 
Edition v1.0.0

(C) Leif Jonsson, 2018

Cover Image (C) Tea Andersson

ISBN 978-91-7685-306-1

ISSN 0345-7524

URL http://urn.kb.se/resolve?urn=urn:nbn:se:liu:diva-147059

Published articles have been reprinted with permission from the respective copyright holder.

Typeset using $\mathrm{X}_{\mathrm{H}} \mathrm{T}_{\mathrm{E}} \mathrm{X}$

Printed by LiU-Tryck, Linköping 2018 
Till min familj 



\section{POPULÄRVETENSKAPLIG SAMMANFATTNING}

I denna avhandling har vi studerat hur man kan använda maskininlärning för att effektivisera felhantering i storskalig mjukvaruutveckling. Felhantering i storskalig mjukvaruutveckling är idag till stor del en manuell process som är mycket kostsam, komplicerad och felbenägen. Processen består i stora drag av att hitta vilket team som skall åtgärda en felrapport och var i mjukvaran som felet ska rättas. Maskininlärning kan förenklat beskrivas som en kombination av datavetenskap, matematik, statistik och sannolikhetslära. Med denna kombination av tekniker så kan man "träna" mjukvara att vidta olika åtgärder beroende på vad man ger den för indata. Vi har samlat mer än 50000 felrapporter från mjukvaruutveckling i svensk industri och studerat hur vi kan använda maskininlärning för att maskinellt kunna hitta var ett fel ska rättas och vilket utvecklingsteam som ska tilldelas felrapporten för rättning. Resultaten visa på att vi kan nå en precision upp till ca $70 \%$ genom att kombinera ett antal standardmetoder för maskininlärning. Detta är i nivå med en mänsklig expert, vilket varit ett viktigt resultat i överföringen av tekniken till praktisk användning. Felrapporter består av både så kallad strukturerad data, som till exempel vilken kund, land, plats osv som felrapporten kommer ifrån, men också så kallad ostrukturerad data som till exempel den textuella beskrivningen av felrapporten och dess titel. Detta har gjort att en stor del av avhandlingen har kommit att handla om hur man kan representera ostrukturerad text i maskininlärningssammanhang. Vi har i avhandlingen utvecklat en metod för att lösa ett problem som ursprungligen formulerades 2003 som kallas Latent Dirichlet Allocation (LDA). LDA är en extremt populär maskininlärningsmodell som beskriver hur man matematiskt i en dator kan modellera teman i text. Detta problem är mycket beräkningskrävande och för att man ska kunna lösa problemet inom rimlig tid så krävs det att man parallelliserar problemet över många datorkärnor. De dominerande teknikerna för att parallellisera LDA har hittills varit approximativa, dvs. inte matematiskt exakta lösningar av problemet. Vi har i avhandlingen kunnat visa att vi kan lösa problemet matematiskt exakt men ändå lika snabbt som de förenklade approximativa metoderna. Förutom den uppenbara fördelen att ha en korrekt modell i stället för en approximation som man inte vet hur stort fel den introducerar, så är det också viktigt när man bygger vidare på LDA modellen. Det finns många andra modeller som har LDA som en komponent och vår metod är applicerbar i många av dessa modeller också. Om man bygger en ny modell baserad på LDA men utan en exakt lösning så vet man inte hur det fel som introduceras med approximeringen påverkar den nya modellen. I värsta fall kan den nya modellen förstärka det ursprungliga felet och man får ett slutresultat som har ett mycket större fel än från början. Även vi har byggt vidare på LDA modellen och utvecklat en ny maskininlärningsteknik som vi kallar DOLDA. DOLDA är en så kallad klassificerare. En klassificerare inom maskininlärning är en metod för att tilldela objekt till olika klasser. Ett enkelt exempel på en klassificerare finns i många e-post programvaror. I detta exempel så kan e-postprogrammet klassificera e-post som endera reklam eller inte reklam. Detta är ett exempel av en så kallad binär klassificering. En mer sofistikerad klassificering kan vara att sortera nyhetsartiklar i olika kategorier som nyheter, sport, underhållning, osv. Den klassificerare som vi utvecklat är helt generell, men vi applicerar den på bugghanteringsproblemet. Vår klassificerare tar både strukturerad data och text som indata och kan tränas genom att köra den på många historiska felrapporter. När DOLDA modellen tränats upp kan den klassificera felrapporter, där kategorierna är de olika utvecklingsteamen i organisationen, eller var i koden en felrapport ska lösas. Vår klassificerare bygger på en statistisk teori som kallas för Bayesiansk statistik. Det finns många fördelar med den Bayesianska statistiken som direkt förs över till vår modell. En av de viktigaste är att man med den Bayesianska metoden alltid får ett mått på osäkerheten i resultaten. Detta är viktigt för att man ska kunna veta hur säker maskinen är på att resultatet är korrekt. Detta gör att om maskinen är osäker på klassificeringen så kan man låta en människa ta över istället, annars kan man låta maskinen utföra åtgärder som till exempel att tilldela en felrapport till ett utvecklingsteam. 


\begin{abstract}
This thesis investigates the possibilities of automating parts of the bug handling process in large-scale software development organizations. The bug handling process is a large part of the mostly manual, and very costly, maintenance of software systems. Automating parts of this time consuming and very laborious process could save large amounts of time and effort wasted on dealing with bug reports. In this thesis we focus on two aspects of the bug handling process, bug assignment and fault localization. Bug assignment is the process of assigning a newly registered bug report to a design team or developer. Fault localization is the process of finding where in a software architecture the fault causing the bug report should be solved. The main reason these tasks are not automated is that they are considered hard to automate, requiring human expertise and creativity. This thesis examines the possibility of using machine learning techniques for automating at least parts of these processes. We call these automated techniques Automated Bug Assignment (ABA) and Automatic Fault Localization (AFL), respectively. We treat both of these problems as classification problems. In ABA, the classes are the design teams in the development organization. In AFL, the classes consist of the software components in the software architecture. We focus on a high level fault localization that it is suitable to integrate into the initial support flow of large software development organizations.
\end{abstract}

The thesis consists of six papers that investigate different aspects of the AFL and ABA problems. The first two papers are empirical and exploratory in nature, examining the ABA problem using existing machine learning techniques but introducing ensembles into the ABA context. In the first paper we show that, like in many other contexts, ensembles such as the stacked generalizer (or stacking) improves classification accuracy compared to individual classifiers when evaluated using cross fold validation. The second paper thoroughly explore many aspects such as training set size, age of bug reports and different types of evaluation of the ABA problem in the context of stacking. The second paper also expands upon the first paper in that the number of industry bug reports, roughly 50,000, from two large-scale industry software development contexts. It is still as far as we are aware, the largest study on real industry data on this topic to this date. The third and sixth papers are theoretical, improving inference in a now classic machine learning technique for topic modeling called Latent Dirichlet Allocation (LDA). We show that, unlike the currently dominating approximate approaches, we can do parallel inference in the LDA model with a mathematically correct algorithm, without sacrificing efficiency or speed. The approaches are evaluated on standard research datasets, measuring various aspects such as sampling efficiency and execution time. Paper four, also theoretical, then builds upon the LDA model and introduces a novel supervised Bayesian classification model that we call DOLDA. The DOLDA model deals with both textual content and, structured numeric, and nominal inputs in the same model. The approach is evaluated on a new data set extracted from IMDb which have the structure of containing both nominal and textual data. The model is evaluated using two approaches. First, by accuracy, using cross fold validation. Second, by comparing the simplicity of the final model with that of other approaches. In paper five we empirically study the performance, in terms of prediction accuracy, of the DOLDA model applied to the AFL problem. The DOLDA model was designed with the AFL problem in mind, since it has the exact structure of a mix of nominal and numeric inputs in combination with unstructured text. We show that our DOLDA model exhibits many nice properties, among others, interpretability, that the research community has identified as missing in current models for AFL. 


\section{ACKNOWLEDGEMENTS}

This research was funded and supported by Ericsson $\boldsymbol{A B}$, but any views or opinions presented in this text are solely those of the author and do not necessarily represent those of Ericsson $\boldsymbol{A B}$

My deepest gratitude to my university supervisors, Professor Kristian Sandahl at Linköping University and Associate Professor David Broman, at the time at Linköping, now at KTH. For any PhD student the advisors have a crucial role, so also for this student. Kristian and David, you have generously allowed me to very freely choose my own path. You have always encouraged me on this path even though it has most certainly diverged from what you expected. Never have you forced your will upon me, but always happily, and engagingly, supported my ideas. Kristian and David, you have formed a perfect complimentary team. Kristian with the wisdom that comes with many years of experience, and David with the vigor and enthusiasm of someone starting their new career. Kristian, thank you for the many encouraging and guiding conversations. David, thank you, for setting the moral standard and expecting nothing less than my best. Thanks also to Dr. Sigrid Eldh, my supervisor at Ericsson. You have always wanted my best. Thank you for your support and encouragement over the years.

In addition to the collaboration with my supervisors I have also been extremely lucky to get to work with some great researchers! I had the great fortune of meeting Dr. Markus Borg at ICSE 2013, and what a refreshing meeting that was! You were as interested in bug reports as I was, and what a great researcher and writer you are. Although, I think that with you talent for words, you should have better appreciated my naming suggestion! Let's keep those curves coming!

A major part of a $\mathrm{PhD}$ undertaking is the intellectual development, and none have had more impact on my intellectual development than my collaborators Dr. Måns Magnusson and Professor Mattias Villani, at the Division of Statistics and Machine Learning at Linköping University. Having spent many years in computer science, of course knowing that it is a superior field compared to statistics, you have actually made me greatly appreciate your field! Mattias, I am forever grateful for letting me take your courses on Bayesian learning in spite of being just a lowly computer scientist. It wasn't easy, but it has expanded my intellectual universe immensely! Your generosity, kindness, humor, and deep knowledge is a constant inspiration. I will let slide, your mockery of Perl... All these attributes are rubbing off on your students. One of them is Måns. Måns, thank you so much for the great collaboration we have had during many years and hopefully many years to come! Late nights, weekends, and weekdays, long discussions and throwing around ideas, it has been great fun, and always energizing! I envy your energy, intellect, humor, and kindness! Likewise, many thanks to Mathias Quiroz for the great 
conversations, jokes, and pleasant company, and for generously sharing your knowledge! With friends and collaborators like you, life is good! Im also very grateful to Professor Thomas Schön for letting me take your excellent course "Machine Learning", in spite of my only real qualification was thinking "math is fun". The course was really a transformative kickstart into the field!

In all of the turmoil of doing a PhD you need friends outside of work to keep you sane, and in touch with the "real world". My profound thanks to my great friend, and infinitely generous teacher Peter Clarke. Our discussions are stimulating, invigorating, and just great fun! It's very hard to think about probabilities when doing Jujutsu. Training and discussing with you has certainly given my brain much needed rest from theory while being focused on Jujutsu practice. My deepest gratitude and admiration also goes to Debbie Clarke, for always making us feel so welcome and taking so good care of us when we come down under to visit. Your fighting spirit and generosity is awe inspiring! My warmest thanks also to Mike and Judy Wallace for your friendship, kindness, and hospitality. Thanks also to all my other Aussie friends, Dale Elsdon, Mike Tyler, Richard Tatnall and the whole of the TJR group in Perth for always being so generous and kind to us when we come down to visit. Many thanks also to my great friends in training, Michael Mrazek, Ricky Näslund and Fredrik "Rico" Blom for your friendship, discussions and fun training.

Another crucial part of life is family. My deepest gratitude to mum and dad for the freedom under responsibility you have given me my whole life. You taught me responsibility and showed me what the power of trust can bring in a person. My dear brother Stefan with his lovely partner Cecilia and their fantastic Anton, it's always fun and relaxing to spend time with you. I'm so glad that you are part of my family. My gratitude also to Pierre, Jennifer, Tuva and Ella for your company, friendship, all the nice dinners and BBQ evenings.

To my great colleagues at Ericsson, thank you all so much for discussions, encouragement, critique and for creating a stimulating environment to work in. Special thanks goes to Roger Holmberg and Mike Williams for believing in me to start with, and giving me this opportunity. Further, during this time I have managed to finish off a row of managers that have all given me support in this endeavor. Thank you Jens Wictorinus, Roger Erlandsson and Magnus Holmberg. Many thanks to Jonas Plantin for stimulating talks and the opportunity to work with, and learn from you in the quality work. Special thanks also to Jan Pettersson, Benny Lennartson, Henrik Schüller, Aiswaryaa Viswanathan and the Probably team for the fun work we do in the analytics area. Thanks also to Andreas Ermedahl and Kristian Wiklund for discussions and support. Thanks to Martin Rydar for fighting the good fight! Keep rocking!

Thanks to Anne Moe at Linköping University for all the help, support, and for keeping everything on track. Thanks to Ola Leifler for help with 
$\mathrm{LAT}_{\mathrm{E}} \mathrm{X}$ when the $\mathrm{AT}_{\mathrm{E}} \mathrm{X}$-panic has set in. Thank you to Mariyam Brittany Shahmehri for proofreading and help making all of this slightly more understandable.

My gratitude also goes to two great Swedish institutions that has made it possible for me to learn, study, and better myself. The Swedish education system, in particular, Linköping University, and Ericsson AB.

Having worked on this dissertation for too many years now, there is a high probability that there is someone I may have forgotten to mention. To you I beg, please accept my sincerest apology for the omission! I promise I will make it up to you in my next dissertation!

Finally, to the best two girls in the world, Tess and Tea, I love you! You are my life. I would surely not have made it through all off this without your support and patience. My deepest gratitude to my wonderful Tess for all the love, support, and encouragement over the years. I couldn't have done it without you dear! Thank you Tea, for being the best daughter any father can have! You are wise, intelligent, kind, and a beautiful person. No words can express the pride I take in being your dad. Thank you also for letting me use your beautiful picture on the cover of this dissertation. My beautiful girls, you have both graciously accepted "I just have to go and work a bit more" for many years. Now we are free!

Leif Jonsson

Hässelby Villastad, Stockholm

April 2018 


\section{Contents}

Abstract $\quad$ V

Acknowledgments $\quad$ ix

Contents $x$

List of Figures xiii

$\begin{array}{ll}\text { List of Tables } & \text { xviii }\end{array}$

$\begin{array}{lr}\text { List of Publications } & 1\end{array}$

Related Publications $\quad 3$

Preface $\quad 5$

$\begin{array}{llr} & \text { Introduction } & \mathbf{7}\end{array}$

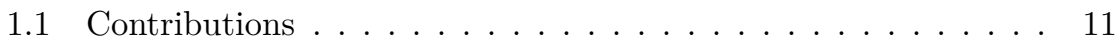

1.2 Thesis Overview . . . . . . . . . . . . . . . . . . 11

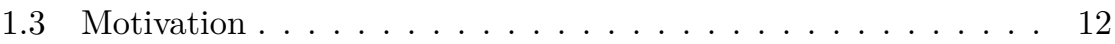

1.4 Research Questions . . . . . . . . . . . . . . . . . . 12

1.5 Personal Contribution Statement . . . . . . . . . . . . . . . 13

1.6 Short Summary of Included Papers . . . . . . . . . . . . . . . 16

1.7 Delimitations . . . . . . . . . . . . . . . . . 17

2 Large-Scale Software Development $\quad 19$

2.1 Large-Scale Development . . . . . . . . . . . . . . . . . . . . . . 19

2.2 Software Development in Industry . . . . . . . . . . . . . . . . 20

2.3 The Tower of Babel . . . . . . . . . . . . . . . . . . 20

2.4 A Bug's Life . . . . . . . . . . . . . . . . . . . . . . . . . . . . . . . 22

2.5 The Bug Tracking System . . . . . . . . . . . . . 24

2.6 Automatic Fault Localization vs. Automatic Bug Assignment . 25

2.7 A Taxonomy of Automatic Fault Localization Scenarios . . . . 26

3 Methodology $\quad 29$

3.1 Method Overview . . . . . . . . . . . . . . . . . 29

3.2 The Author's Personal Experience . . . . . . . . . . . . . 29 
3.3 Methods Used . . . . . . . . . . . . . . . . . . . . . . . . . 30

3.4 Paper I . . . . . . . . . . . . . . . . . . . . . . . . . 33

3.5 Paper II . . . . . . . . . . . . . . . . . . . 34

3.6 Paper III . . . . . . . . . . . . . . . . . . . . . . . 34

3.7 Paper IV . . . . . . . . . . . . . . . . . . . . . . . . . . 35

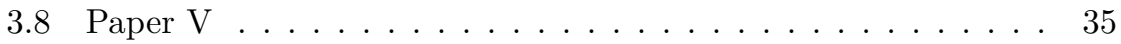

3.9 Paper VI . . . . . . . . . . . . . . . . . . . . . . 35

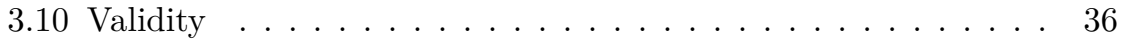

4 Theory 41

4.1 Machine Learning Introduction . . . . . . . . . . . . . . . 42

4.2 Probability Reminder . . . . . . . . . . . . . . . . . . . 42

4.3 What is Learning? . . . . . . . . . . . . . . . . . . . . . . . . . 49

4.4 Bayesian Learning . . . . . . . . . . . . . . . . 67

4.5 Visualization . . . . . . . . . . . . . . . 96

5 Results and Synthesis $\quad 99$

6 Discussion and Future Work 105

6.1 Aspects of Industrial Adoption . . . . . . . . . . . . . . 105

6.2 Recommendations for an Updated Software Development Process 107

6.3 Boundary Between ABA and AFL . . . . . . . . . . . . 108

6.4 Hierarchical Classification . . . . . . . . . . . . . . . . . . . . 109

6.5 Other Contextual Information . . . . . . . . . . . . . . 110

6.6 An End-to-end View of Automated System Analysis . . . . . 111

7 Conclusion 113

$\begin{array}{ll}\text { Bibliography } & 115\end{array}$

8 Paper I 123

8.1 Introduction . . . . . . . . . . . . . . . . . . . . 124

8.2 The Anomaly Report process _ . . . . . . . . . . . 125

8.3 Approach . . . . . . . . . . . . . . . . . . . 128

8.4 Prototype Implementation and Evaluation . . . . . . . . . . 135

8.5 Results and Analysis . . . . . . . . . . . . . . . . . . 138

8.6 Related Work . . . . . . . . . . . . . . . . . . . . . . . . . 142

8.7 Conclusion . . . . . . . . . . . . . . . . . . . . . . . . 143

References . . . . . . . . . . . . . . . . . . . 143

9 Paper II 149

9.1 Introduction . . . . . . . . . . . . . . . . 150

9.2 Machine Learning . . . . . . . . . . . . . . . . . . . . 152

9.3 Related Work on Automated Bug Assignment . . . . . . . . . 154

9.4 Case Descriptions . . . . . . . . . . . . . . . . . . 162

9.5 Method . . . . . . . . . . . . . . . . . . . 166

9.6 Results and Analysis . . . . . . . . . . . . . . . . . . . . 180

9.7 Threats to Validity . . . . . . . . . . . . . . . . . . . . . 189

9.8 Discussion . . . . . . . . . . . . . . . . . . . . . 193 
9.9 Conclusions and Future Work _ . . . . . . . . . . . . . 197

References . . . . . . . . . . . . . . . . . . . . 199

10 Paper III 209

10.1 Introduction . . . . . . . . . . . . . . . . . . . . . 209

10.2 Related work . . . . . . . . . . . . . . . . . . 212

10.3 Partially Collapsed sampling for topic models . . . . . . . . . . 214

10.4 Experiments . . . . . . . . . . . . . . . . . . 219

10.5 Discussion and conclusions . . . . . . . . . . . . . . 229

References . . . . . . . . . . . . . . . . . 2230

10.A Proofs . . . . . . . . . . . . . . . . . 2234

10. B Variable selection in $\Phi$. . . . . . . . . . . . . . . . . 235

10.C Algorithms . . . . . . . . . . . . . . . . 238

11 Paper IV 245

11.1 Introduction . . . . . . . . . . . . . . . . . . . . . 245

11.2 Related work . . . . . . . . . . . . . . . . . . 248

11.3 Diagonal Orthant Latent Dirichlet Allocation . . . . . . . . . . 249

11.4 Inference . . . . . . . . . . . . . . . . . . . . 251

11.5 Experiments . . . . . . . . . . . . . . . . . . 255

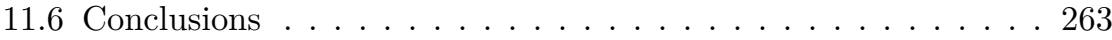

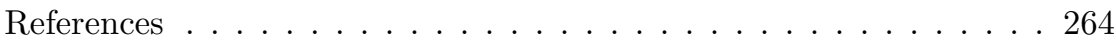

11.A Derivation of the MCMC sampler . . . . . . . . . . . 267

11.B Efficient updating of supervision effects . . . . . . . . . 270

12 Paper V 275

12.1 Introduction . . . . . . . . . . . . . . . . . . . . . 275

12.2 Deployment Scenario . . . . . . . . . . . . . . . . . . 278

12.3 Experimental Setup . . . . . . . . . . . . . . . . . . . 285

12.4 Results and Discussion _ . . . . . . . . . . . . . . 286

12.5 Related Work . . . . . . . . . . . . . . . . . . . . . . 291

12.6 Conclusion . . . . . . . . . . . . . . . . . . . . . . . . . 292

References . . . . . . . . . . . . . . . . . 292

13 Paper VI 297

13.1 Introduction . . . . . . . . . . . . . . . . . 297

13.2 Previous Work . . . . . . . . . . . . . . . . . 300

13.3 The Algorithm . . . . . . . . . . . . . . . . . . . . . 301

13.4 Performance Results . . . . . . . . . . . . . . . . . . . . 307

13.5 Discussion . . . . . . . . . . . . . . . . . . . 311

References . . . . . . . . . . . . . . . . . . . 314

13.A Appendix A: Efficiency of Collapsed and Uncollapsed Gibbs

Samplers . . . . . . . . . . . . . . . . . 317

13.B Appendix B: Poisson-Pólya Urn Asymptotic Convergence . . . 318 


\section{List of Figures}

2.1 A simplified visualization of a software bug's life. . . . . . . 22

4.1 t-SNE rendering of a bug database using unstructured text via LDA, using the individual document topic mixture as input

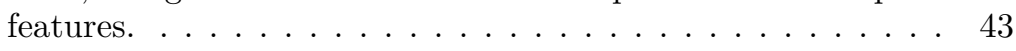

4.2 Discrete probability distribution. . . . . . . . . . . . 44

4.3 Continuous probability distribution. . . . . . . . . 45

4.4 The probability of a continuous probability distribution is the area under the curve of the continuous probability distribution, not the y value of the pdf! . . . . . . . . . . . 46

4.5 Histogram of 800 draws from a normal distribution with the true normal overlayed. . . . . . . . . . . . . . . . . 47

4.6 Scatter plot of data in Table 4.1. . . . . . . . . . . 53

4.7 Plot of the sum of squares error (SSE) as a function of the slope. We see that there is one point where the error is at a minimum and we know that this is a global minimum since the error is a quadratic function. We know from calculus that the slope of the tangent (i.e. derivative) at the minimum of a quadratic function equals zero. . . . . . . . . . . . . . . 54

4.8 Plot of data in Table 4.1 with various fits. The solid red line is the "real line" that was used to simulate data from. The purple dotted line is the regression line of a single variable linear model without intercept, and the green dash-dotted line is the regression line of a model fitted with an intercept. 4.9 t-SNE rendering of a bug database using the unstructured text encoded as LDA topic distributions as inputs. The color represents the class of the bug report. . . . . . . . . . 97

6.1 Hierarchical Classification. The F-level could be function level, S - subsystem level, C - component level and, B - block level. Histograms indicate probability per level in the tree. . . 109

6.2 There are often many sources that, taken together, could help identify the component that is most likely contain a bug. . . . 110

6.3 End-to-end view of automated system analysis . . . . . . . . 112

8.1 AR Life Time . . . . . . . . . . . . . . . . . . . . . 125

8.2 Simplified AR Hierarchy $\ldots \ldots \ldots \ldots$ 
8.3 Stacked Generalizer implemented by a Bayesian Network . . . 130

8.4 Stacked Generalizer with Soft Evidence . . . . . . . . . . . . . 134

8.5 Class diagram of implemented tool . . . . . . . . . . . . . 136

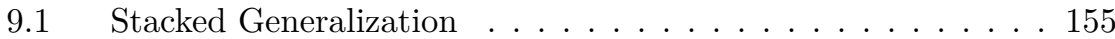

9.2 Techniques used in previous studies on ML-based bug assignment. Bold author names indicate comparative studies, capital $\mathbf{X}$ shows the classifier giving the best results. IR indicates Information Retrieval techniques. The last row shows the study presented in this paper. . . . . . . . . . . . . . 157

9.3 Evaluations performed in previous studies with BTS focus. Bold author names indicate studies evaluating general purpose ML-based bug assignment. Results are listed in the same order as the systems appear in the fourth column. The last row shows the study presented in this paper, even though it is not directly comparable. . . . . . . . . . . . . . . . . . 159

9.4 A simplified model of bug assignment in a proprietary context.165

9.5 The prediction accuracy when using text only features ("textonly") vs. using non-text features only ("notext-only") . . . . 172

9.6 Overview of the controlled experiment. Vertical arrows depict independent variables, whereas the horizontal arrow shows the dependent variable. Arrows within the experiment box depict dependencies between experimental runs A-E: Experiment A determines the composition of individual classifiers in the ensembles studied evaluated in Experiment B-E. The appearance of the learning curves from Experiment $\mathrm{C}$ is used to set the size of the time-based evaluations in Experiment D and Experiment E. . . . . . . . . . . . . . . . . . 173

9.7 Overview of the time-sorted evaluation. Vertical bars show how we split the chronologically ordered data set into training and test sets. This approach gives us many measurement points in time per test set size. Observe that the time between the different sets can vary due to non-uniform bug report inflow but the number of bug reports between each vertical bar is fixed. . . . . . . . . . . . . . . . . . . . . 178

9.8 Overview of the cumulative time-sorted evaluation. We use a fixed test set, but cumulatively increase the training set for each run. . . . . . . . . . . . . . . . . . . . 180 
9.9 Comparison of BEST (black, circle), SELECTED (red, triangle) and WORST (green, square) classifier ensemble. . . . . . 183

9.10 Prediction accuracy for the different systems using the BEST (a) WORST (b) and SELECTED (c) individual classifiers under Stacking . . . . . . . . . . . . . . . . . . . . 184

9.11 Prediction accuracy for the datasets Automation (a) and Telecom 1-4 (b-e) using the BEST ensemble when the time locality of the training set is varied. Delta time is the difference in time, measured in days, between the start of the training set and the start of the test set. For Automation and Telecom 1,2 , and 4 the training sets contain 1,400 examples, and the test set 350 examples. For Telecom 3, the training set contains 619 examples and the test set 154 examples.

9.12 Prediction accuracy using cumulatively (farther back in time) larger training sets. The blue curve represents the prediction accuracy (fitted by a local regression spline) with the standard error for the mean in the shaded region. The maximum prediction accuracy (as fitted by the regression spline) is indicated with a vertical line. The number of samples (1589) and the accuracy $(16.41 \%)$ for the maximum prediction accuracy is indicated with a text label $(\mathrm{x}=1589 \mathrm{Y}=16.41$ for the Automation system). The number of evaluations done is indicated in the upper right corner of the figure (Total no. Evals). . . . . . . . . . . . . . . . . . . . 188

9.13 This figure illustrates how teams are constantly added and removed during development. Team dynamics and BTS structure changes will require dynamic re-training of the prediction system. A prediction system must be adapted to keep these aspects in mind. These are all aspects external to pure ML techniques, but important for industry deployment. . . . . . . 190

9.14 Perceived benefit vs. prediction accuracy. The figure shows two breakpoints and the current prediction accuracy of human analysts. Adapted from Regnell. . . . . . . . . . . . . . . . . 196

10.1.1 The generative LDA model. . . . . . . . . . . . . . . . . . 210

10.4.1 Average time per iteration (incl. standard errors) for Sparse AD-LDA and for PC-LDA using the Enron corpus and 100 topics. . . . . . . . . . . . . . . . . . . . . . . .

10.4.2 Log marginalized posterior for the NIPS dataset with $K=20$
(upper) and $K=100$ (lower) for AD-LDA (left) and PC-LDA

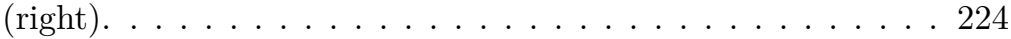

10.4.3 The sparsity of $n^{(w)}$ (left) and $n^{(d)}$ (right) as a function of cores for the NIPS dataset with $K=20$ (upper) and $K=100$ (lower). . . . . . . . . . . . . . . . . . 2225

10.4.4 Log marginal posterior by runtime for PubMed $10 \%$ (left) and PubMed 100\% (right) for 10, 100, and 1000 topics using 16 cores and 5 different random seeds. . . . . . . . . . . . 226 
10.4.5 Log marginal posterior by runtime for Wikipedia corpus (left) and the New York Times corpus (right) for 100 topics using 16 cores. . . . . . . . . . . . . . . . . 227

10.4.6 Log marginal posterior by runtime for the PubMed corpus for 100 topics (left) and 1000 topics (right) using sparse PC-LDA. 228

10.B.1 Log marginalized posterior for different values of $\pi$ for PubMed 10\% (left) and NIPS (right). . . . . . . . . 237

11.3.1 The Diagonal Orthant probit supervised topic model (DOLDA)249

11.5.1 Accuracy of MedLDA, taken from Zhu et al. 2012 (left) and accuracy of DOLDA for the 20 Newsgroup test set (right). . . 257

11.5.2 Accuracy for DOLDA on the IMDb data with normal and Horseshoe prior and using a two step approach with the Horseshoe prior. . . . . . . . . . . . . . . . . 258

11.5.3 Coefficients for the IMDb dataset with 80 topics using the normal prior (left) and the Horseshoe prior (right). . . . . . . 258

11.5.4 Coefficients for the genre Romance in the IMDb corpus with 80 topics using the Horseshoe prior (upper) and a normal prior (below)

11.5.5 Regression coefficients for the class Crime for the IMDb corpus with 80 topics using the Horseshoe prior (upper) and a normal prior (below). . . . . . . . . . . . . . . . 260

11.5.6 Document entropy (left) and topic coherence (right) for the IMDb corpus. . . . . . . . . . . . . . . . . . 261

11.5.7 Coherence and document entropy by supervised effect with 50 topics. . . . . . . . . . . . . . . . . . 262

11.5.8 Scaling performance (left) and parallel performance (right). The scaling experiments were run for 5,000 iterations and the parallel performance experiments were run for 1,000 iterations each. All were run with 3 different random seeds and the average runtime was computed. In the parallel experiment, the $20 \%$ NYT Hierarchical data was used.

12.2.1 $\beta$ coefficients for the Core.Networking component. The Core.Networking component has five signal variables, Z11, Z27, Z28, Z55 and Z82 which represents topics 11, 27, 28,

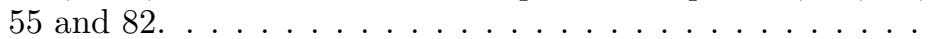

12.2.2 Probability distribution over the classes with very low uncertainty. . . . . . . . . . . . . . . . . 280

12.2.3 Probability distribution over the classes with comparatively high uncertainty. . . . . . . . . . . . . . . . . 281

12.2.4 Deployment use-case. . . . . . . . . . . . . . . . . . . . 282

12.2.5 $\beta$ coefficients for the Core.Security component. The Core.Security component has five signal variables. . . . . . . . 284 
12.4.1 Precision vs. accuracy and precision vs. acceptance rate plots from five experimental runs (folds) on the Mozilla dataset. The Horseshoe prior and 100 topics are used. The top graph shows that as the uncertainty in the prediction decreases (prediction precision increases) the prediction accuracy increases. Bottom graph shows that as we increase the required precision in the classification, more classifications are rejected and the ratio of accepted classifications decreases. . . . . . . . . 287

12.4.2 Comparison of the effect on the $\beta$ coefficients when using the Horseshoe (a) prior vs the normal (b) prior for the Mozilla class (component) Core.Networking. On the $\mathrm{X}$-axis is the variable of the corresponding $\beta$ coefficient. The value of the $\beta$ coefficient is on the Y-axis. . . . . . . . . . . . . . 289

13.1.1 Directed acyclic graph for LDA. . . . . . . . . . . . . . . . 298

13.4.1 Log-posterior trace plots for standard partially collapsed LDA and Pólya Urn LDA, on a runtime scale (hours). Dashed line indicates completion of 1,000 iterations. . . . . . . . . . . . 308

13.4.2 Log-posterior trace plots for standard partially collapsed LDA and Pólya Urn LDA, on a per iteration scale. . . . . . . . . . . 309

13.4.3 Left: runtime for $\boldsymbol{\Phi}$ and $\boldsymbol{z}$ for Pólya Urn LDA, as a percentage of standard partially collapsed LDA - lower values are faster. Right: percentage of runtime taken by $\boldsymbol{\Phi}$ and $\boldsymbol{z}$ for Pólya Urn LDA. . . . . . . . . . . . . . . . . . . . . . 309

13.4.4 Runtime and convergence for Pólya Urn LDA, Partially Collapsed LDA, Fully Collapsed Sparse LDA, and Fully Collapsed Light LDA, on the NYT corpora. . . . . . . . . . . . . . 309

13.4.5 Left: test set log-likelihood for Pólya Urn LDA and Partially Collapsed LDA. Right: test set topic coherence for Pólya Urn LDA and Partially Collapsed LDA. . . . . . . . . . . . . . . 310

13.4.6 Left-most three plots: runtime for Pólya Urn LDA, Partially Collapsed LDA, and Fully Collapsed Sparse LDA on a single core. Right-most two plots: runtime for Pólya Urn LDA versus number of available CPU cores. . . . . . . . . . . . . . . . 310

13.4.7 Runtime for Pólya Urn LDA for various rare word thresholds, vocabulary sizes, and data sizes. . . . . . . . . . . . . 310

13.A.1 Trace plots for the collapsed and uncollapsed Gibbs samplers for a $T$ distribution on $\mathbb{R}^{2}$ with $\rho \in\{0.9,0.99,0.999\}$, together with target distributions. In 25 iterations, the uncollapsed Gibbs sampler has traversed the entire distribution multiple times, whereas the collapsed Gibbs sampler has not done so even once, covering increasingly less distance for larger $\rho$. . . 318 


\section{List of Tables}

3.1 Mapping of each paper to research method and purpose. . . . 32

3.2 Mapping of paper to research method, goals, and main evaluation metric. .................. 33

4.110 observations of the real-valued variable $y$ given inputs $x \quad$. 52

4.210 observations of the binary-valued variable $y$ given inputs $x \quad 61$

4.3 A simplified imaginary database of bug reports. Ellipsis at the end of descriptions symbolizes that a longer text follows.

4.4 Summary of bug reports from Table 4.3. The numbers in the cells reports the total number of bug reports from each site on each component. In this example Site 2 has reported 26 bugs on the Controller component. . . . . . . . . . . . . . 64

4.5 Class probabilities in percent (not summing to 100 due to rounding) using Naive Bayes fitted using MLE and Laplace Smoothing (Equation number in parenthesis). . . . . . . . 67

4.6 A simplified imaginary database of bug reports. Ellipsis at the end of descriptions symbolizes that a longer text follows. $\quad 87$

8.1 Subset of AR fields used in our research . . . . . . . . . . . 126

8.2 Example Conditional Probability table of Naive Bayes (NB) node in Fig. 8.3 . . . . . . . . . . . . . . . . . . . . . . . 132

8.3 Example Conditional Probability Table of Soft Evidence node in Fig. 8.4 . . . . . . . . . . . . . . . . . . . . 135

8.4 Prediction Rates depending on model . . . . . . . . . . . . . 139

8.5 Prediction Rates by humans . . . . . . . . . . . . . . . . 139

8.6 Classifier results . . . . . . . . . . . . . . . . . . . 141

9.1 Overview of the research questions, all related to the task of automated team allocation. Each question is listed along with the main purpose of the question, a high-level description of our study approach, and the experimental variables involved. 167 
9.2 Datasets used in the experiments. Note: At the request of our industry partners the table only lists lower bounds for Telecom systems, but the total number of sums up to an excess of 50,000 bug reports. . . . . . . . . . . . . . . . 168

9.3 Features used to represent bug reports. For company Telecom the fields are reported for Telecom 1,2,3,4 respectively. . . . . 170

9.4 Individual classifiers available in Weka Development version 3.7.9. Column headings show package names in Weka. Classifiers in bold are excluded from the study because of long training times or exceeding memory constraints. . . . . . . . . 175

9.5 Individual classifier results (rounded to two digits) on the five systems use the full data set and 10-fold cross validation. Out of memory is marked O-MEM and an execution that exceeds a time threshold is marked O-TIME. . . . . . . . . . . 181

10.1 LDA model notation. . . . . . . . . . . . . . . . . . . 210

10.2 Summary statistics of training corpora. . . . . . . . . . . 220

10.3 Mean inefficiency factors, IF, (standard deviation in paren-

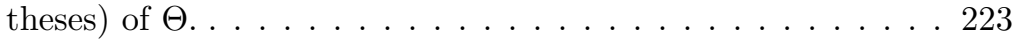

10.4 Mean inefficiency factors, IF, (standard deviation in parentheses) of $\Phi \ldots \ldots$. . . . . . . . . . . . . . . 223

10.5 Runtime to reach the high density region for 100 and 1000

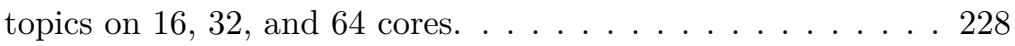

10.6 Variable selection of $\Phi \ldots \ldots$. . . . . . . . . . . 237

11.1 DOLDA model notation. . . . . . . . . . . . . . 250

11.2 Corpora used in experiment, by the number of classes $(L)$, the number of documents $(D)$, the vocabulary size $(V)$, and the total number of tokens $(N)$. Statistics have been computed using the word tokenizer in the tokenizers $\mathrm{R}$ package with default settings (Mullen, 2016). . . . . . . . . . 256

11.3 Top words in topics using the Horseshoe prior. . . . . . . . . . 259

12.1 Top words for signal topics (Z11, Z27, Z28, Z55 and Z82) for the class Core.Networking from the Mozilla dataset. The topic label is manually assigned. . . . . . . . . . . . . . . 279

12.2 Dataset statistics. Vocab. size is the size of the vocabulary of the unstructured data after stop word and rare word trimming.285

12.3 Dependent and Independent variables per dataset. . . . . . . . 286 
12.4 Summary of prediction accuracy experiments. We present the same figures for 40 and 100 topics for Stacking+TF-IDF because there is no notion of topics in that method, the $(*)$ is a reminder of this. Figures in parentheses are the accuracies at minimum uncertainty in the prediction (which means a low acceptance rate, see Figure 12.4.1) . . . . . . . . . . . . . . 290

13.1 Notation for LDA. Sufficient statistics are conditional on algorithm's current iteration. Bold symbols refer to matrices, bold italic symbols refer to vectors. . . . . . . . . . . . . . . . 298

13.2 Corpora used in experiments. . . . . . . . . . . . 308 


\section{List of Publications}

[I] Leif Jonsson, David Broman, Kristian Sandahl, and Sigrid Eldh. "Towards Automated Anomaly Report Assignment in Large Complex Systems Using Stacked Generalization". In: Software Testing, Verification and Validation (ICST), 2012 IEEE Fifth International Conference on. Apr. 2012, pp. 437-446.

[II] Leif Jonsson, Markus Borg, David Broman, Kristian Sandahl, Sigrid Eldh, and Per Runeson. "Automated bug assignment: Ensemble-based machine learning in large scale industrial contexts". English. In: Empirical Software Engineering 21 (4 Aug. 2016), pp. 1533-1578. ISSN: 1382-3256.

[III] Måns Magnusson, Leif Jonsson, Mattias Villani, and David Broman. "Sparse Partially Collapsed MCMC for Parallel Inference in Topic Models". In: Journal of Computational and Graphical Statistics (July 2017).

[IV] Måns Magnusson, Leif Jonsson, and Mattias Villani. "DOLDA - A Regularized Supervised Topic Model for High-dimensional Multi-class Regression". In: Revision resubmitted to Journal of Computational Statistics (June 2017).

[V] Leif Jonsson, David Broman, Måns Magnusson, Kristian Sandahl, Mattias Villani, and Sigrid Eldh. "Automatic Localization of Bugs to Faulty Components in Large Scale Software Systems using Bayesian Classification". In: Software Quality, Reliability and Security (QRS), 2016 IEEE International Conference on. IEEE. Aug. 2016, pp. 423-430.

[VI] Alexander Terenin, Måns Magnusson, Leif Jonsson, and David Draper. "Polya Urn Latent Dirichlet Allocation: a doubly sparse massively parallel sampler". In: Accepted for publication in IEEE Transactions on Pattern Analysis and Machine Intelligence. 2018. 



\section{Related Publications}

[1] Markus Borg and Leif Jonsson. "The More the Merrier: Leveraging on the Bug Inflow to Guide Software Maintenance". In: Tiny Transactions on Computer Science 3 (2015). 



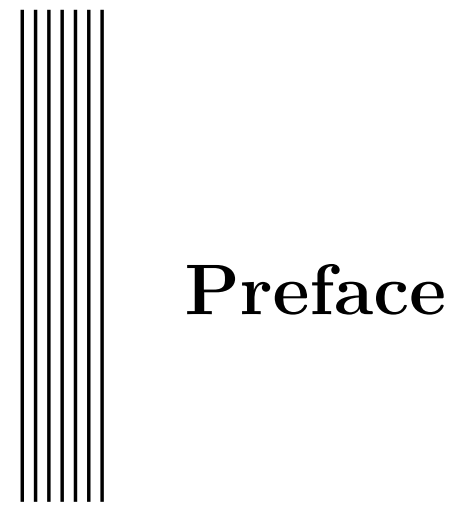

This thesis concerns the partial automation of the assignment and analysis stages of the bug handling process in large software development projects. The impetus for the work came mainly when a high level manager at Ericsson declared one day that "Faults should be found in one day!" This was a provocative statement since the typical "time to find a fault," as it is called at Ericsson, was not one day... I liked the idea, but thought; Why one day? Why not one second?! Of course, for this to be feasible, the whole bug analysis process would have to be changed and automation support for these processes would be needed. This work is a combination of empirical and theoretical research. The empirical work has mainly been performed at my "day job" at Ericsson $\mathrm{AB}$ with the purpose of exploring and improving large-scale software development practices. The goal of the theoretical work is to implement improvements that have been identified as necessary in the methods explored in the empirical research. 



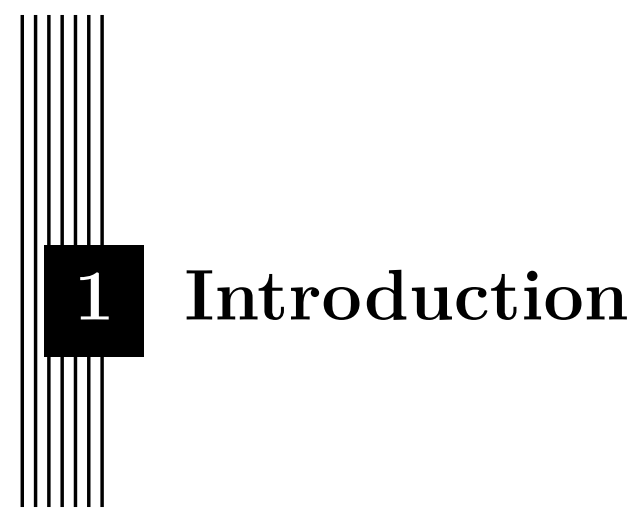

The core area of interest in this thesis is the efficient and effective handling of bugs in large scale systems development. The word bug is commonly used in modern society; many of our everyday devices and software seem plagued by them, but what are bugs? The IEEE Standard Classification for Software Anomalies [1] (IEEE-1044) defines nomenclature standards for talking about software anomalies. The IEEE-1044 starts by stating that

"The word 'anomaly' may be used to refer to any abnormality, irregularity, inconsistency, or variance from expectations. It may be used to refer to a condition or an event, to an appearance or a behavior, to a form or a function. The 1993 version of IEEE Std $1044^{T M}$ characterized the term 'anomaly' as a synonym for error, fault, failure, incident, flaw, problem, gripe, glitch, defect, or bug [emphasis added], essentially deemphasizing any distinction among those words."

We note here that the words "anomaly" and "bug" can be seen as synonymous. The terms defined in IEEE-1044 are, in short:

1. defect: An imperfection or deficiency in a work product where that work product does not meet its requirements or specifications and needs to be either repaired or replaced. NOTE: Examples include such things as 1) omissions and imperfections found during early life cycle phases 
and 2) faults contained in software sufficiently mature for test or operation.

2. error: A human action that produces an incorrect result.

3. failure: (A) Termination of the ability of a product to perform a required function or its inability to perform within previously specified limits. (B) An event in which a system or system component does not perform a required function within specified limits. NOTE: A failure may be produced when a fault is encountered.

4. fault: A manifestation of an error in software.

5. problem: (A) Difficulty or uncertainty experienced by one or more persons, resulting from an unsatisfactory encounter with a system in use. (B) A negative situation to overcome.

For the main part of this thesis, we will not differentiate between the nuances of these concepts. We will simply talk about bugs. We will sometimes say things along the lines of "...the bug is located in...", instead of the longer "...the fault which is the underlying cause of the bug is located in...".

Wong, Gao, Li, Abreu, and Wotawa [2], in their survey of more than 300 papers on Software Fault Localization, similarly use the words "fault" and "bug" interchangeably.

In this thesis, bugs are the manifestation of faults in the software that appear as annoyances to the end-user. In the telecommunications context, an end-user can be a large telecommunication provider or a user of a so-called "smart phone". The bugs are reported by the end-user to the developer of the software in the form of bug reports, which are written summaries of bugs. ${ }^{1}$ Two of the main tasks in dealing with bugs are bug assignment, also called bug triaging, and fault localization. Bug assignment is the process of assigning the investigation of a bug report to a design team or individual developer. Fault localization is the process of finding the location of the underlying fault of the bug report in the software code. Collectively, we call these two tasks bug handling. Handling of bug reports is the main focus of this thesis.

We also want to point out that the IEEE-1044 talks about software anomalies, but we, in general, discuss large-scale system development. Here, system refers to the combination of both hardware and software. Telecommunications systems are one example of system development, and so are industrial robotics systems. In development of large-scale systems, the border between software and hardware faults is getting more and more blurred due to the increased integration of software into modern hardware. This does not have a large impact on our discussion, so we will not emphasize it in this text. But it is worth noting that in our case, bugs can also be caused by hardware failures.

\footnotetext{
${ }^{1}$ Bug reports are also sometimes referred to as anomaly reports or issues.
} 
The last decades have seen immense progress in the software development field, especially in recent years with the mostly ubiquitous use of structured testing practices. The front-runner of these testing practices is unit testing, which is tightly integrated with software development. In spite of improved testing practices, software development advancements and huge amounts of research in the field of software defect prevention, the software industry are still producing bugs en masse. That the production is faster and lower in cost provides little solace. Bugs are a fact that the software industry must deal with. The handling of bugs generally falls under what is called software maintenance. Software maintenance is a large part of the total cost of software development. Ideally, the software would be produced (from a perfect requirement specification) and then deployed to the customer, and it should work perfectly. Aside from the design, no additional cost, would be expended. Sadly, the state-of-practice is not so. At the core of the maintenance process is the detection of anomalies in the behavior of the system, handling of bug reports, analysis of the bug reports, fixing the offending code, testing of the new release and re-delivery of the software to the end-user. Advances in software development processes have automated substantial parts of this chain. For example, large parts of test and delivery are automated. But as Wiklund points out [3], there are still challenges in the test automation area. The notable exceptions to routine automation are the bug routing, analysis, and fixing stages.

The aim of this thesis is to develop theories and techniques to make fault localization and bug assignment effective in the context of large-scale industrial software development. Ultimately, we argue that these theories could significantly lower the cost of bug assignment and fault localization, which are both major parts of the costly software maintenance process in large-scale software development.

The main hypothesis of this work is that the most efficient way to increase the effectiveness and efficiency in the bug handling tasks is to increase the level of automation. However, several aspects of the bug handling problem are, by their nature, not directly amenable to traditional automation approaches. Traditional automation consists of a series of deterministic steps that can be scripted by a computer program. But reading a bug report and figuring out which development team should handle the bug, or where the corresponding fault is located in the software, includes several aspects that is currently not possible to automate with traditional deterministic automation techniques. The main reason for this is that the knowledge representation in the vast majority of bug reporting tools is not designed for machine consumption. Bug reports are typically written in prose by humans, for human consumption. This fact makes it hard for machines to deterministically interpret and infer the underlying semantics of a bug report. Another exacerbating reason is that deducing where a bug is located, and which teams are best equipped to deal 
with the problem, often requires implicit knowledge or information that is not readily available.

Having established that the bug handling problem is hard to solve with deterministic techniques, we turn to non-deterministic i.e., probabilistic techniques. Machine Learning (ML) techniques are inherently probabilistic and very well-suited to non-deterministic problems. Hence, our aim is to explore, if and how, we can use machine learning techniques to automate bug assignment and fault localization. A subgoal of this thesis is to explore current methods and improve on existing methods to overcome these obstacles to automation.

Automatic Fault Localization (AFL) and Automatic Bug Assignment $(\mathrm{ABA})$ are what we will call the strategies for increasing efficiency in the bug report handling part of the software development process. The main strategy we explore in this thesis for automating fault localization and bug assignment is the use of machine learning.

Bug reports consist of both structured and unstructured data. So when using ML to implement AFL and ABA, a large part of the problem concerns how to best exploit the unstructured text content of the bug reports. This makes dealing with, and representing, unstructured text in ML one of the core areas of focus for this thesis.

A vast amount of research has focused on fault localization techniques, but the majority of this research has been done on what we call low level fault localization. What we mean by "low level", is that the user of the technique needs to have access to both the full source code and an executable binary to use the technique. Sometimes, in addition to both binary and source code, test cases must also be run towards the binary. In other cases, a program model is needed. Examples of these types of techniques include spectrum based techniques, slice based techniques, and model based techniques. Of all the papers in the study by Wong, Gao, Li, Abreu, and Wotawa [2], 74\% fall into one of these categories.

By contrast, in our research, we focus on what we call high level fault localization. I.e., the user of the technique does not need access to source code, test cases, a binary executable or any sort of model (UML or other) of the code. We focus mainly on only the information that can be extracted from bug reports and possibly surrounding static information. This could be historical test case verdicts, high level models, or configuration information, for example.

In no way should this be taken as an indication that low level techniques are not important or needed. On the contrary, both approaches complement each other and can and should be used together. We see our research as a much-needed complement to low-level techniques of AFL. 


\subsection{Contributions}

The main contributions in this thesis are threefold. On the empirical side, we have studied the problems of ABA and AFL in a large-scale industrial context through three papers, Paper I, Paper II, and Paper V. This is much needed since the vast majority of papers on AFL and ABA are studied in an Open Source Context. Our studies are based on a large amount of real world industry bug reports. The conclusions from these studies are that ML is a very promising approach for $\mathrm{ABA}$ and $\mathrm{AFL}$ in industry contexts. We introduce an ML technique called Stacking in the context of ABA and AFL and show that it consistently gives good accuracy. But we also conclude that the "black box" approach of Stacking can be limiting. In cases where a richer model is desired, we propose to use Bayesian methods for ABA and AFL. We conclude that the Bayesian approach gives a rich model for organizations that feel the need for more sophisticated models. The final choice of technique will depend on the priorities of the organization.

Our second main contribution is on the theoretical side in Papers III, IV, and VI, in the area of Topic Modeling. Topic Modeling is a probabilistic technique for modeling topics or themes, mainly in text, but it has also been applied to images. The most popular topic modeling technique is called Latent Dirichlet Allocation (LDA) [4]. We have studied inference in the LDA model as implemented by Markov Chain Monte Carlo (MCMC). There we have presented theoretical proofs, and shown empirically, the efficiency of a understudied class of MCMC samplers. This class of samplers, called partially collapsed samplers, have not been extensively studied in the Topic Modeling community. We derive exact samplers for the most popular Topic Modeling method and show that it is as fast and efficient as current state-ot-the-art approximate samplers. Our results are contrary common beliefs in the Topic Modeling community.

Our third main contribution is the implementations of our samplers and tools that we have made available for industry, and the research and ML community as Open Source Software.

\subsection{Thesis Overview}

This thesis consists of the papers listed in the List of Publications and the following chapters, one through seven. After this introductory and motivating chapter, we discuss the context of large-scale software development in Chapter 2. Next is Chapter 3, which deals with methodological concerns. The Method chapter is then followed by a Theory chapter (Chapter 4). The theory chapter is written with the interested computer scientist in mind as an introductory text that will hopefully introduce some machine learning theory to be helpful when reading the included papers. The theory chapter in turn, is followed by the Results and Synthesis in Chapter 5 and the Discus- 
sions and Future work in Chapter 6. The dissertation is then concluded with Conclusions in Chapter 7 and the included papers.

\subsection{Motivation}

According to Wong, Gao, Li, Abreu, and Wotawa [2], fault localization " $i s$ widely recognized to be one of the most tedious, time consuming, and expensive yet equally critical activities in program debugging".

The bug assignment problem is identified as one of the main challenges in change request $(\mathrm{CR})$ management in a large systematic research mapping study by Cavalcanti, Mota Silveira Neto, Machado, Vale, Almeida, and Meira $[5]$.

Furthermore, in a 2002 study report [6] the National Institute of Standards and Technology (NIST) estimated that "the annual costs of an inadequate infrastructure for software testing is estimated to range from \$22.2 to \$59.5 billion". With our continuously increasing dependency on software, it is unlikely that this cost has decreased. In the report, an important factor for driving down the cost of testing is locating the source of bugs faster and with more precision.

We have thus identified bug handling as an expensive and labor-intensive task. This makes the lack of supporting tools in the area of automatic fault localization and bug assignment, in the context of large-scale industrial system development, the main motivation for this work

There are two more motivations that drive this dissertation. First, from a customer relations perspective, it is often crucial to quickly understand the cause of a bug and any further possible (adverse) manifestations of the bug beyond what has already been observed. Once this is understood, there might be possible workarounds to mitigate the problems caused by the bug. It is not always necessarily crucial to actually correct the fault very quickly, but the customer wants to understand the problem and its implications quickly. Speed in the fault localization process leads to better customer relations for any company. Second, we want to minimize waste in the bug handling process. Waste consists of humans performing repetitive, mundane, error-prone and laborious tasks. The more we can automate the bug handling process, the more efficient and less wasteful we can make it.

\subsection{Research Questions}

The main research questions that drive this thesis are the following:

1. RQ1: How well, in terms of accuracy, can we expect machine learning techniques to perform? Is it feasible to replace human bug assignment and fault localization with machine learning techniques in large scale industry SW development projects? 
2. RQ2: Which machine learning techniques should be used in large-scale industry settings?

3. RQ3: How can we improve ML techniques, aside from increasing prediction accuracy, to increase their usefulness in AFL and ABA?

Having identified a need for automation and concluded that machine learning is a suitable candidate approach, we need to investigate the feasability of this approach; this is the scope of RQ1. RQ1 is mainly investigated and answered in Papers I and II and V.

Research question RQ2 deals with the question of which techniques are most suitable in our context and for our problem. Different contexts will generally have different requirements. For instance, it is not obvious that machine learning techniques that have been optimized for robotics and computer vision are suitable for bug handling. RQ2 asks which considerations and requirements need to be considered for bug handling. This question is answered in Papers I, II and V.

The final research question, RQ3, builds on RQ2 and asks how we can improve ML techniques to better fulfill the requirements we identified in RQ2. This question is mainly answered in Papers III and IV, VI.

\subsection{Personal Contribution Statement}

Most contemporary research projects are collaborative efforts where the teams and collaborators are invaluable parts in the effort. This research is no different. Below we detail the personal contributions of the author to this thesis.

\section{Paper I - "Towards Automated Anomaly Report Assignment in Large Complex Systems Using Stacked Generalization" - Leif Jonsson, David Broman, Kristian Sandahl, and Sigrid Eldh}

Leif introduced the idea of using stacked generalization and ensemble techniques for bug assignment. Leif extracted, prepared, and validated the data set, designed and implemented the automated machine learning tool. Most of the analysis was done by Leif. The other authors supported with discussions and some analysis. Leif wrote most of the paper. 


\section{Paper II - "Automated bug assignment: Ensemble-based machine learning in large scale industrial contexts" - Leif Jonsson, Markus Borg, David Broman, Kristian Sandahl, Sigrid Eldh, and Per Runeson}

Leif proposed and initiated the extensive follow up study to Paper I. Leif identified, acquired, extracted, and validated, four of the five datasets in the paper. Leif identified and designed the feature set to be used in the data. Leif designed and implemented a new automatic machine learning tool from the ground up. Leif and Marcus designed the study, analyzed the results and wrote the paper together with support and discussions with the other authors.

\section{Paper III - "Sparse Partially Collapsed MCMC for Parallel Inference in Topic Models" - Måns Magnusson, Leif Jonsson, Mattias Villani, and David Broman}

Leif and Måns introduced the idea of a correct parallel LDA sampler based on the independence of variables in probabilistic models. Måns derived the theoretical basis for a correct parallel sampler for the Latent Dirichlet Allocation (LDA) model. Leif extracted and prepared the data, and designed and implemented, the sampler including parallelization and optimization. Leif and Måns drove the design of the study and analyzed the results in discussions with the other authors. Måns and Leif wrote the paper together.

\section{Paper IV - "DOLDA - A Regularized Supervised Topic Model for High-dimensional Multi-class Regression" - Måns Magnusson, Leif Jonsson, and Mattias Villani}

Leif and Måns proposed the idea of a supervised Latent Dirichlet Allocation model augmented with additional covariates. Måns derived the theoretical basis for the sampler and led the design and analysis of the study with help from Leif and the other authors. Leif extracted and prepared the data for the experiments. Leif designed and implemented the sampler. Måns organized the writing process and wrote most of the final text, which was reviewed and commented on by Leif and the other authors. 


\section{Paper V - "Automatic Localization of Bugs to Faulty} Components in Large Scale Software Systems using Bayesian Classification" - Leif Jonsson, David Broman, Måns Magnusson, Kristian Sandahl, Mattias Villani, and Sigrid Eldh

Leif suggested using a supervised Latent Dirichlet Allocation model augmented with additional covariates for Automatic Fault Localization. Leif and Måns drove the design of the study in discussions with the other authors. Leif extracted and prepared the data for the experiments. Leif designed and wrote the code for all approaches compared in the paper and ran the experiments. Leif led the design of the study and analysis of the results with contributions from David and Mattias. Leif wrote most of the final text with feedback from David Broman.

\section{Paper VI - "Polya Urn Latent Dirichlet Allocation: a doubly sparse massively parallel sampler" - Alexander Terenin, Måns Magnusson, Leif Jonsson, and David Draper}

Alexander and Måns, suggested using the Polya Urn approximation of the Dirichlet distribution in LDA. Alexander and Måns developed the theoretical details of the sampler in discussions with the other authors. Leif, Måns and Alexander discussed and prototyped implementation details and Leif designed and implemented the final code in the previous package developed for Partially Collapsed LDA. The final text was mainly written by Alexander and was reviewed and commented on by Leif and the other authors.

\section{Open Source Software Published}

As part of the papers mentioned above, we have also produced four software libraries that are released as Open Source and are publicly available at GitHub.

1. http://github.com/lejon/PartiallyCollapsedLDA - Fast Partially Collapsed Gibbs samplers for LDA (Java)

2. http://github.com/lejon/DiagonalorthantLDA - Bayesian Supervised classification based on LDA (Java)

3. http://github.com/lejon/T-SNE-Java - $\mathrm{T}$ Distributed Stochastic Neighbour Embedding - t-SNE (Java)

4. http://github.com/lejon/TSne.jl - T Distributed Stochastic Neighbour Embedding - t-SNE (Julia) 
While none of our research directly relates to t-SNE, its implementation was an important part of creating the tool-set used in the research. Empirical research places high requirements on the tools used. t-SNE has been an important tool in our exploratory data analysis.

\subsection{Short Summary of Included Papers}

To facilitate reading some of the introductory text without having to read the full papers, we give a short summary of the included papers.

Paper I explores the possibility of using Machine Learning (ML) to solve the problem of ABA in large-scale industrial software development. An initial study is made on real industry data using a machine learning technique called Stacked Generalization or Stacking. The study concludes that the ML techniques achieve an accuracy comparable to that of humans, thus demonstrating that using ML for ABA is feasible.

Paper II is an extension of Paper I, but with a wider scope and deeper analysis. In Paper II we study more than 50,000 bug reports and more classification techniques. The conclusions from Paper I are strengthened and a methodology for selecting classifiers is presented. A deeper study of the characteristics of stacking, its accuracy under different conditions, and advice for industrial adoption is presented.

Paper III attacks a problem from Paper II: how to accurately and efficiently represent unstructured text in machine learning contexts. We derive a fast and mathematically correct Markov Chain Monte Carlo (MCMC) sampler for the LDA model. This is in contrast to the prevailing approximate parallel models, which are not proper LDA samplers. Using measurements on standard evaluation datasets, we show that it achieves competitive performance compared with other state-of-the-art samplers that are not mathematically correct representations of the LDA model. We have further extended this work in Paper VI to an even faster sampler based on a Polya-Urn model [7].

Paper IV extends the unsupervised LDA to a supervised classification technique we call DOLDA, which, besides text, can additionally incorporate structured numeric, and nominal covariates. DOLDA then fulfills the original requirement from Paper III of a classifier which not only incorporates both text and structured covariates and reaches sufficient prediction accuracy, but which also generates a rich output for further analysis. The richness in the model output is due to DOLDA being a fully Bayesian technique.

Paper $\mathbf{V}$ applies the classifier in Paper IV to the original problem of AFL. It shows that with a Bayesian approach, we can get a classifier which both achieves a high degree of accuracy and is highly flexible in use, and also gives highly interpretable results. We show that using the inherent quantification of uncertainty in Bayesian techniques, an organization can flexibly trade the level of automation against desired prediction accuracy in the AFL problem. 
Paper VI improves on the LDA sampler in Paper III by adding sparsity to a dense matrix data structure in the LDA model typically named $\phi$. This update gives three concrete benefits; it vastly speeds up the sampling of the $\phi$ matrix by the introduced sparsity and the sparsity reduces the memory requirements for storing $\phi$. Another important improvement is the speed of sampling the so called topic indicators, the other main data structure in the LDA model. Improving the speed in sampling the topic indicators is very beneficial since this is where the bulk of the sampling effort is expended. We show in the paper that although the introduced sparsity comes from approximating the Dirichlet distribution using a Polya Urn, the approximation error vanishes with data size.

\subsection{Delimitations}

This work applies to large-scale software development, with very large code bases and many developers. The approach is unlikely to be efficient for small scale software development where the problem of routing and locating faults in the code is substantially simpler than in a large-scale setting. The approach further assumes that there is a reasonable amount of decent quality training data available for training the ML system. We have not seen any studies that indicate any concrete numbers of developers or bug reports at which an ML approach to fault localization and bug triaging starts to be efficient. In Paper II we suggest as a starting recommendation that around 2000 bug reports should be available. A further rough guideline is that the number of developers should probably at least be in the hundreds and the code base should be in the hundreds of thousands of lines of code. Another aspect of this limitation is that we focus on high level fault localization. By high level fault localization, we mean localizing a bug report to a higher level than what is typically done in traditional automatic fault localization research. Traditionally, localizing the fault to a single line or statement is typically the goal. In this thesis we focus on component level or higher. The level of detail will be dictated by the size of the software and organization. Our focus should in no way be taken as a statement that traditional low level fault localization is unimportant!

Since the work is focused on the industry context, it is unclear if the benefits of our suggested approach have the same value in an Open Source Software (OSS) context. However research [8, 9, 10] using similar approaches in an OSS context indicates that this is the case.

In this thesis we have limited ourselves to investigating ML-based classification techniques for AFL and ABA. There are other possible approaches, such as Information Retrieval techniques [11, 12, 13, 14]. It is likely that a combination of the two approaches is the best option in a full deployment 
scenario. Exactly how the combination would best be designed should be studied further.

ML-based classification implicitly also incurs another limitation; classifiers typically cannot handle very large numbers of classes. In our studies, the largest number of classes we have dealt with is 118 . While this is obviously not enough to represent the full details of a large, complex software system, we argue that for team assignment or component fault localization, it should be sufficient at the very least for a first analysis. For more detailed classification, a hierarchical approach should probably be employed, probably in combination with other techniques such as Information Retrieval methods. We suggest that such a combination approach would be a very interesting field for further study.

We also limit ourselves to mostly studying the technical aspects of AFL and ABA. We do not explore processes around introducing and using MLbased techniques in an organization. When introducing ML techniques into an organization, new ways of working will have to be introduced. New ways of working will, in turn, create other challenges. These challenges are not studied in this thesis, although we have ongoing discussions with our industry partner on the topic. In Paper II we give some recommendations for deployment based on findings in the paper. In most deployment scenarios, we see that the traditional ways of working will continue in parallel, with new ways of working for some time to be able to evaluate how well an ML-based approach works in a particular setting. 


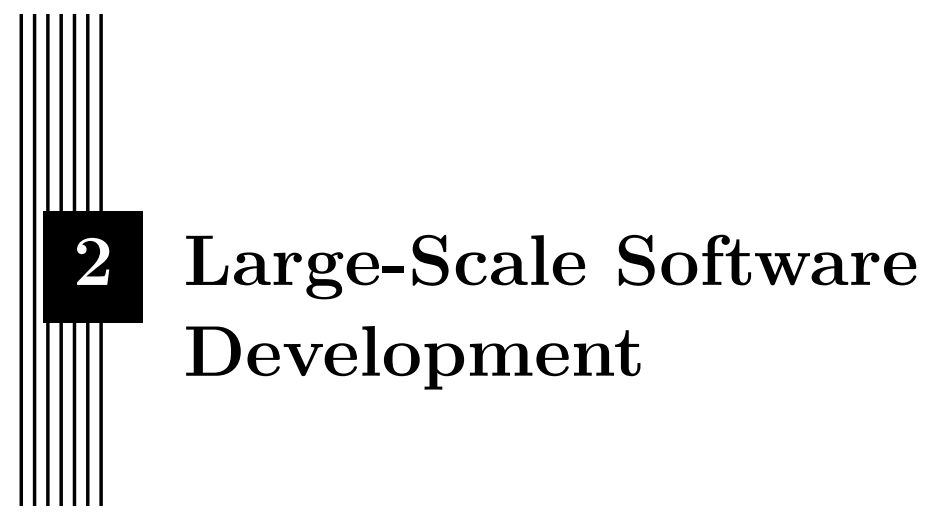

The context of this research is large-scale industrial software development, and the research questions come from problems in this context. In this section we describe some of the characteristics of this context to give a better understanding of some of the problems that arise in this type of environment. The context is also an important basis for the goals of the techniques that we study in this thesis.

\subsection{Large-Scale Development}

Not all development is, or has to be large-scale. In this thesis, we give a simple example of large-scale software development as having certain characteristics. The example we use is not the only definition of what large scale is, nor is it anywhere close to a complete one, but it will suffice for our purposes. In our context, large-scale manifests itself in two main aspects; the size of the development organization, and the size and complexity of the software that is being developed.

A large-scale software development organization has on the order of thousands of developers. This often means that all developers of the product are not located in the same building, or even in the same city. The organization might even be geographically distributed over several countries, continents, and time zones, where the teams do not share the same culture or mother tongue. 
Large scale products are several million lines of code in size, with many subsystems, application layers, and an almost endless variation of configurations. Complexity means that the product involves complex protocol stacks, standards, and both specific hardware solutions and different programming languages. Longevity of a large-scale product means that the lifetime of the product covers decades. This means that a product and its features have evolved over many years, and older parts (hardware and software) of the product have to work together with newer versions.

\subsection{Software Development in Industry}

One of the main aspects of the context relevant to this thesis is the development unit of interest. Almost irrespective of the concrete development process, the unit of interest in large-scale industrial software development is the team rather than individual developers. This is one aspect where industrial development is typically different from development contexts where the focus is on individual developers. The reason for focusing on the team, rather than individual developers, is that there are just too many developers to allow for keeping track of each individual for product management. When a team is assigned a task, the team itself is responsible for solving the task at hand. In this way, the team will solve the daily work of organizing individual tasks, taking into consideration individual developers' areas of expertise or other deciding factors such as people being sick, on vacation, involved in other projects, or absent for other reasons.

While cross-functional teams can, in principle, work with all aspects of the product, it is still common to have a sort of organizational separation of concerns. Typically in large-scale development, there is a support organization, a development organization, and possibly a services organization. These organizations work on the same products but with different responsibilities. Although this separation of concerns allows for easier management of a large organization, it also creates organizational gaps between the staff in the different organizational units.

\subsection{The Tower of Babel}

The industrial large-scale context described above leads to several practical problems, many related to the coordination of teams. In very large organizations, teams can be separated in many aspects. Holmström et al. [15] describe some of the effects of three types of distances that they refer to as geographical, temporal, and cultural. Jaanu et al. [16] extend this with a fourth category that they call the organizational distance, which can affect global software development companies such as, for example, Ericsson. 
These distances all make it hard to coordinate work efficiently. Jaanu et al. [16] stress that "communication across organizational boundaries will benefit from tools supporting both efficient information sharing and building common understanding". This thesis is a response to the lack of tools in the areas of bug triaging and fault localization for large-scale organizations. A large organization allows for parallel development, but at the same time, the scale also makes it notoriously difficult to coordinate all aspects of the development process between all of the teams and individuals.

Bjarnason et al. [17] describe some of the challenges of aligning requirements engineering with verification and validation. One of the main challenges that they identify is Cooperating Successfully, where clear agreement and communication and organisational boundaries are major aspects of the challenge. It is notable that Company F, in Bjarnason's account, which is similar to our context in terms of size and domain, exhibits all but one, of the 16 (10 main challenges and 6 subcategories) identified challenges. Many of them, including

- Aligning goals and perspectives

- Cooperating successfully

- Defining clear and verifiable requirements

- Defining complete requirements

- Defining a good verification process

- Maintaining alignment when requirements change

- Managing a large document space

deal essentially with communication issues. The communication challenge is further expanded on by Sabaliauskaite et al. [18] who report: "In a large company, gaps in communication across different organizational units often occur, especially at the high level". In the same work, one of the main categories of challenges is Organization and Processes Related Issues.

These challenges manifest themselves in the bug handling process in largescale organizations in several ways. The first point of contact for a bug report may reside in another organization, and/or in another country, than that of the development organization. It is also highly unlikely that the first receiver of a bug report knows the details of the code or even how to access the code that is causing the bug. This renders AFL and ABA techniques, which rely on the first receiver of the bug report running low-level test cases and lowlevel debugging, impossible to use in our context. The organizational gap is just too big to efficiently bridge in the bug triaging stage for techniques that require low-level information. 


\subsection{A Bug's Life}

The life of a software bug is slightly different from the source of its name, the insect. In this thesis, we will simplify the description for ease of presentation. This simplified scheme is visualized in Figure 2.1. In this description we introduce a set of phases that a bug report goes through. This description aims to be simple and generic with the understanding that in reality it is typically much more complex. Also, we do not imply that there are fixed boundaries between the phases, rather, in reality they intertwine. Below we briefly describe the various phases that we will use in the further discussion.

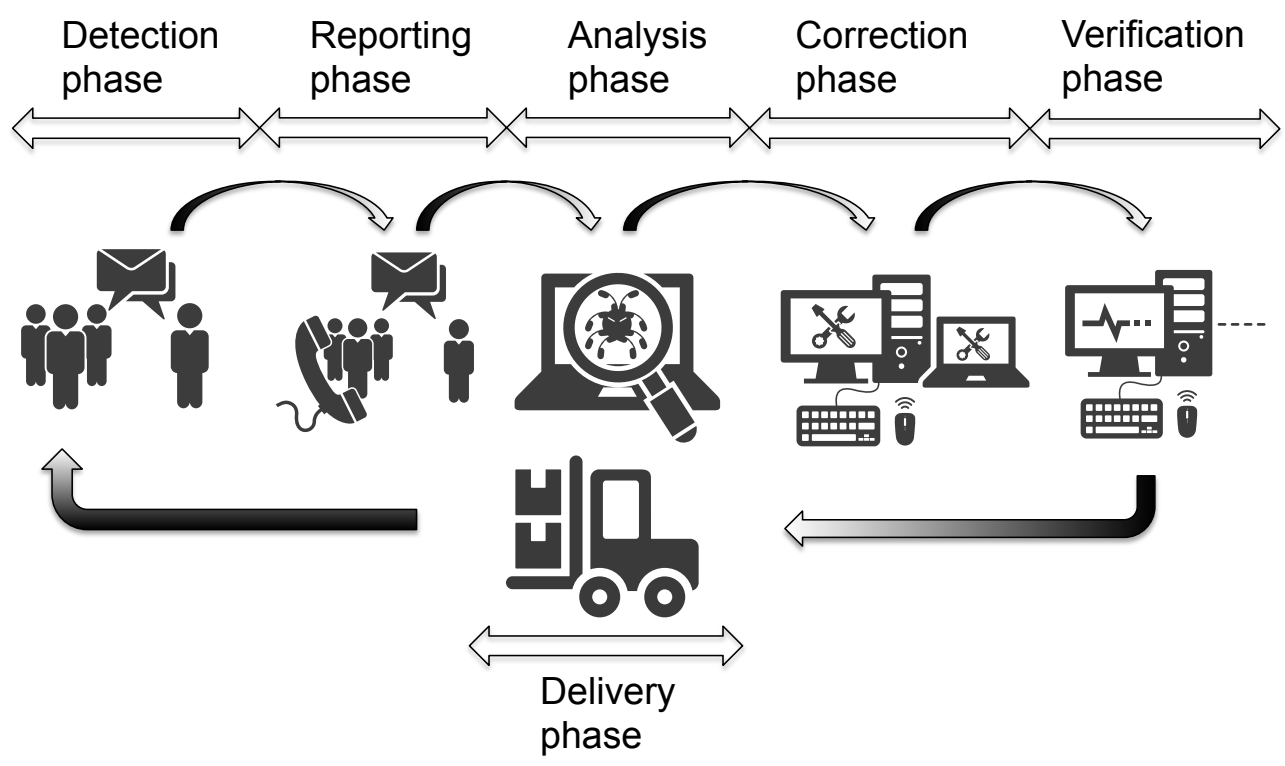

Figure 2.1: A simplified visualization of a software bug's life.

Detection phase: This is the stage where the bug is first discovered. This can occur in several phases of the software development cycle, with an associated cost for each phase. Detection at the customer site is typically the worst and most expensive place to detect a bug. It is better to detect the bug during the development phase, in developer unit/component tests, or during continuous integration, system test, or in acceptance testing [19].

Reporting phase: In the reporting phase, an entity (a developer, tester, or customer) suspects that a bug has been observed. In this phase, initial observations of the bug are gathered and an initial bug report is established. Typically, the procedure for reporting bugs is different if it is reported by an external customer, as opposed to being reported by the software product's own development organization. For customer reported bugs, larger organizations typically have a support organization dedicated to handling customer support 
issues. This organization will enforce certain quality requirements on the bug report. If, on the other hand, the bug is reported by the internal organization, it is typically up to the individual designer or tester to ensure the quality of the bug report. It is up to the reporting entity to come up with a short descriptive slogan for the bug and some descriptive text. In addition to this unstructured data, additional structured data is typically reported. The exact details of this additional data will differ depending on context but typically include

- which customer reported the bug

- which site was running the product where the bug was detected

- which revision of the software was running

- which operating system or architecture was running the software

- additional attachments such as screen shots

- $\log$ files

- support personnel discussions and other supporting material

A very important aspect of this phase, especially with individually reported bugs, is that the analysis phase will be much faster if the quality of the bug report is good. There are huge gains to be made by ensuring high quality bug reports [20].

Analysis phase: This is the phase we focus on in this work. The analysis phase consists mainly of two aspects. The first is assigning the bug report to a person or team for analysis, and the second is locating where in the software architecture the bug is located. A complicating factor for us is that these aspects are intertwined. To know where to send a bug report, the support personnel must know the functional area in which the bug resides, but to know this they must talk to the development teams in the correct area. But of course, finding this team was the original problem. This conundrum is typically solved by layering the system into a hierarchy. The support personnel can then send the bug report to the top level organization of an area, which is responsible for routing the bug report further down in the organization. Bug assignment is thus a multi-stage process. In large development organizations, the corresponding support organizations are typically organized into tiers. Typical names for these tiers are first line, second line and third line support. Even with this tiered approach, it is typically hard for the support organization to know exactly to which team a bug report should be assigned for analysis. One solution to this problem is to have an intermediary entity in the later support lines that is closer to the development organization. This entity can route the bug report to the development team that is most likely 
to be able to find the cause of the bug and fix the offending code. This entity is sometimes called a change control board $(\mathrm{CCB})$ or triager, i.e. someone equipped to triage the bug report to the best person or team. At this triaging stage, the bug report is inspected for clues that might indicate where the report should go. If the report is written on a particular product or a lower level component, it makes the job easier for the triaging entity. Otherwise, typically the triager must read the report and make a best estimate from experience of where to assign the report. In summary, bug assignment and triaging occurs on multiple levels in large organizations.

Correction phase: In this phase it is concluded that a fault is in fact present in the system, and a decision has been made that it should be fixed (this is not the case for all bugs). At this stage, ideally we want to re-use all information that has been gathered during the analysis phase. There is usually a "notebook" or "discussion" or some sort of "history field" for this purpose. Here, information gathered throughout the bug handling process can be stored. If the same person that did the analysis of the bug is also the one fixing the bug, the information and knowledge gathered during the analysis phase is trivially re-used. But even having the same person fixing the bug is not a guarantee of complete information re-use. Sometimes the correction is delayed for a long time and the person cannot remember the analysis when it is time for the correction.

Verification phase: During verification, the software is tested to make sure that fixes to the code have not introduced new faults or destroyed old functionality. Other aspects that are tested are robustness, stability, and interoperability with other parts of the system.

Delivery phase: At this point, the new software is ready to be delivered to the end customer. The delivery phase consists of many administrative steps, such as packaging and version management of the new delivery.

\subsection{The Bug Tracking System}

To manage the work of handling bugs, some sort of Bug Tracking System (BTS) is used. In principle, the BTS can be anything from a whiteboard with sticky notes, to a spreadsheet, to a full-fledged commercial product that is specially designed for tracking bugs. Examples of bug tracking systems are the open source system called Bugzilla, the commercial system Jira, Trac, and IBM ClearQuest. Jira, Trac, and ClearQuest actually support more processes than just bug tracking. For instance, sometimes the bug handling process is separated from the change request process and both Jira and ClearQuest support this. In addition to open and commercially available tools, there are also proprietary, in-house BTS's.

For all of the BTS's we have studied, there are some common key characteristics. A BTS maps the phases of a bug report to states in the BTS. 
The names of the states differ depending on the bug tracking system. But the general scheme contains states for representing a newly created bug, an assigned, or in progress bug, and a finished bug. Typically, there is also some way to indicate how the bug was solved. Not all bugs can be solved-in fact a substantial number of bugs cannot be reproduced or solved [21]. In this case, the bug will be left unsolved with some indication in the BTS that the bug cannot be reproduced or is invalid. To track individual bugs, there is typically an Id field that is unique to each bug report. In Bugzilla, this field is called bug_id and in Jira it is called the key. Both tools have a short text field, typically a one sentence description, that is used as a slogan or summary of the bug report. In Bugzilla, this is called short_desc and in Jira it is called Summary. In the Ericsson in-house tool it is called Heading. In addition to the short slogan, the tools have various support for longer types of unstructured text. The texts can be of various sorts. In Bugzilla, there is a generic field called long_desc, of which there can be several. Typically, the first of these is a longer description of the bug, while the following ones are used for a general discussion of the bug. In Jira, there is a Description field that is used as a longer description of the bug, and separate Comments fields for discussing the bug report. Similarly, in Ericsson's in-house tool, there is a Description field and separate Notes that can be attached to discuss the bug report. In the Ericsson tool there is also a History field, which makes it possible to track the states the bug report has been in.

The information in the BTS is not only text, but typically a combination of nominal, ordinal, numeric, and unstructured text data. For instance, customer, site, or country are examples of possible nominal attributes. Examples of numeric attributes are release and priority. Note that priority could also be ordinal if, instead of a numeric value, it is designated "low", "medium", or "high".

\subsection{Automatic Fault Localization vs. Automatic Bug Assignment}

As we have noted before, bug assignment and fault localization are interconnected. Here we discuss some of the connections between the two, and how they relate to an organization's ways-of-working.

If the development organization is structured so that one particular development team always handles bug reports on one particular subsystem or component, the bug triager is in essence performing fault localization. This is because there is a one-to-one mapping between design team and component, or subsystem. This structuring of the development organization is not necessarily always the case, so it is still valid to separate the two activities. In fact, the opposite is likely to be the more common case, i.e., teams maintaining several subsystems and/or components. Since we believe the latter to be more 
common, we also consider the task of fault localization to be more important than that of bug assignment. We argue that if we solve the fault localization problem, it is a fairly straightforward task to solve the bug assignment problem. If the members of a team always work on the same components, they can easily self-assign bugs if they know which component is at fault.

Alternatively, a simple mapping from a component to a design team can be established. In this way, other external organizations can automatically assign bugs to teams, given that it is known in which component a bug is located. However, the converse is not necessarily true. If a team handles several components it is not obvious where in the software architecture the bug is located, even if one knows which team should handle the bug. Thus, we argue that the problem of automatic fault localization is more interesting than the problem of automatic bug assignment, since the solution to the first problem also, in general, solves the other.

The way-of-working in the organization will also dictate which activity of ABA or AFL is most suitable for the particular organization. For instance, in agile organizations, a top-down assignment of bug reports might not fit with the agile workflow. Hence, ABA may not be applicable. In Agile development, where teams are themselves responsible to pull bug reports from a backlog, an AFL approach makes sense since it can be used at $1^{\text {st }}$ line support to give a first classification of a bug report. This pinpointing of a bug to a component can then be a guide to which bug reports might be suitable for a particular team. Conversely, if the way-of-working dictates that the support organization assigns bug reports top-down, then an ABA methodology is best-suited. Of course, nothing prevents a combined approach! It is certainly possible to do both ABA and AFL, and get the benefits of both if this aligns with the organization's way-of-working.

\subsection{A Taxonomy of Automatic Fault Localization Scenarios}

Automatic Fault Localization can be useful in several different contexts in the software development life-cycle. Some of these contexts include:

1. Development debugging - This is the situation where the software is being developed and some problem has been found in unit tests or lower level function tests that needs to be localized. Here, the individual software developer typically has access to low level details about the whole system, such as source code and low level unit tests. In this context, detailed fault localization techniques, such as spectrum-based approaches [22], are suitable since they require access to the lowest level of detail of the system and the ability to run code and test cases. 
2. Post-test debugging - In large-scale software development, not all testing is done by the individual software developer, but also by dedicated test teams. This typically includes system-wide tests, such as robustness, stability, or integration tests that require specialized knowledge, hardware, and system configuration and setup skills not possessed by all developers. The post-test debugging context is when a bug has been reported by a test team to the development team. In this context, developers, again, typically have access to lower levels of detail of the system, such as the exact configuration, and detailed logs of when and under which circumstances the problem occurred. Developers also typically have access to unit tests and source code of the system in this context.

3. Post-delivery debugging - This is the context where the full system has been delivered to the customer and the customer has reported a bug on a full production system running in live operation. In this context, it is typically not feasible for the first receiver of a bug report to set up the system for detailed troubleshooting. Also, the receiver does not have access to the source code or unit level tests. The first receiver of the bug report has to make a judgment based only on the information that is available in the bug report and his or her own experience to determine where in the architecture the bug is most likely to be located, and where to route the bug report for further troubleshooting.

In this thesis, we focus on the post-delivery debugging context. This is not to say that the other contexts are not important, or that our techniques are not applicable to these contexts. The reason for our focus is mainly due to the relatively small amount of research done in this context and the specific motivations for this thesis. 



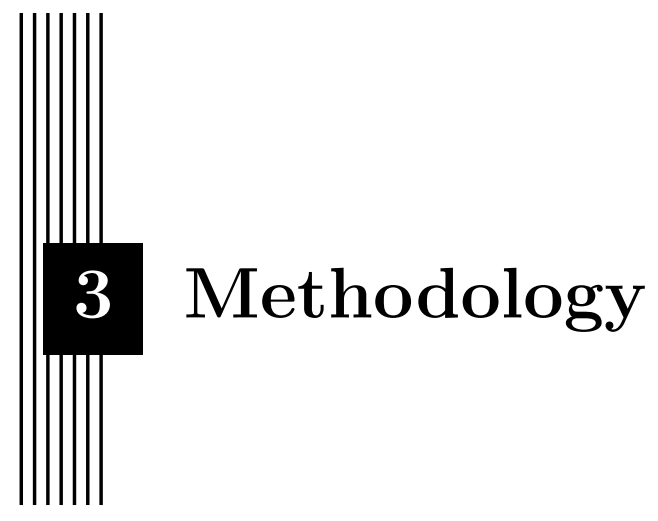

\subsection{Method Overview}

In this chapter we present methodological concerns of this thesis. We first give a background of the author's personal experience, which we feel has played an important role in shaping the material for this thesis. After this introduction, we break down the different methods that have been used and how that maps to the different papers. We finish the chapter by discussing concerns of validity.

\subsection{The Author's Personal Experience}

The work in this thesis has been driven by concrete industry problems that the author has wrestled with in various ways and in various capacities during 20 years in a large-scale software development organization. The author has personally worked extensively with the bug handling process at Ericsson AB. The author's experiences with bug handling come from working in many different capacities. The first experiences were gained in the capacity of being a developer using the system in his daily work for many years. During this time the author was often involved in discussions, decisions, and management fora around bug handling in his capacity as developer, team lead, and assistant project manager. The author has also worked as a test coordinator, helping the test and design teams deal with bug handling processes and the daily work of solving bug reports. Additionally, the author was part of a team that 
phased out an old bug tracking tool to the use of the current main tool for bug handling at Ericsson. This was done for one of the largest Ericsson development organizations. At the same organization, the author has also been part of a team responsible for dealing with efficiency and improvements in the bug handling tools, and ways of working.

\subsection{Methods Used}

We use the classification scheme by Wohlin, Runeson, Host, Ohlsson, Regnell, and Wesslen [23] to describe the methodologies used in our papers. The methods are

- Scientific - the world is observed and models are built based on observations, for example, simulation models.

- Engineering - the current solutions are studied and changes are proposed and then evaluated.

- Empirical - a model is proposed and evaluated through empirical studies, for example, case studies or experiments.

- Analytical - a formal theory is proposed and then compared with empirical observations

We have employed two main research approaches during the work on this thesis. The main approach has been empirical, where we have studied processes, methods, and techniques in a real-world setting. The second main approach has been analytical, where we have mathematically derived algorithms and have proved certain properties of these algorithms.

The purpose is classified using the strategies detailed by Runeson, Host, Rainer, and Regnell [24]. From Runeson, these are:

- Exploratory - finding out what is happening, seeking new insights, and generating ideas and hypotheses for new research.

- Descriptive - portraying the current status of a situation or phenomenon.

- Explanatory - seeking an explanation for a situation of a problem, mostly but not necessarily, in the form of a causal relationship.

- Improving - trying to improve a certain aspect of the studied phenomenon.

The employed method for most of the research in this thesis has been an empirical approach, where the researcher has been working in the real-world environment under study. This type of research setting is sometimes called in-vivo [42]. The main purpose of the research has been two-fold; exploratory 
and improving. Initially we wanted to explore the feasibility of the ideas we had for improving the efficiency in the bug handling process. Once this was established in Paper I and Paper II, we wanted to further improve on the available approaches. This was done in Paper V with the theoretical Papers III, IV and VI as a basis.

Since a lot of the work has been done in-vivo and under Non-Disclosure Agreements (NDAs) we have tried to strike a balance between the restrictions of the NDAs and the desirability of replicable research. Wohlin, Runeson, Host, Ohlsson, Regnell, and Wesslen [23] differentiate between close or exact replication and differentiated replication. In close replications, the original procedures and the original sample population are available and a very detailed replication can be achieved. This is highly desirable, as it helps to build confidence in the outcome. In differentiated replications, the same research questions are studied, but the experimental procedures or the exact population can differ. Even though differentiated replications are not as exact as close replications, much knowledge can be gained from differentiated studies (Wohlin, Runeson, Host, Ohlsson, Regnell, and Wesslen [23, p. 20]). In cases where we have been prohibited by NDAs from publishing our data, we have described the methods in detail to facilitate differentiated replication. In the theoretical work, we have used publicly available datasets for our studies to facilitate close replication. In the last empirical Paper V, we have used both proprietary and publicly available data sets to both cater to the desire to study the applicability in the industrial context, and to facilitate close replication by other academics who do not have access to the proprietary data. We have also published the major part of the code developed during this thesis as Open Source to further increase transparency and facilitate replication (see Section 1.5). This include code for new methods and have extensible examples for ease of use. In Paper I and Paper II, and in part in Paper V, we have traded close replication for differentiated replication to get highly relevant industry results.

We argue that even where close replication is not possible, we get a transfer effect of the knowledge that was gained because we have ensured that the methods and data that were used are applicable to large-scale software development contexts. This has been achieved by using generally available software libraries with general purpose machine learning techniques. We have avoided developing highly specialized solutions for our particular contexts by selecting features that are highly general and available in all of the bug tracking systems that we have come across. We have validated this by using both open source datasets and data from different industry partners.

High quality empirical research places high requirements on the instrumentation in the tools used. In an effort to facilitate closely replicable research, we have implemented all of the code in our theoretical papers from scratch in the same framework as the one in which the original comparison techniques were implemented. We have also re-implemented other comparison approaches in 
the same framework to be able to measure performance in detail, to the extent that it is possible. Also, in the more empirical work, we have implemented the code for instrumentation in the same language as the libraries that were used in order to do the instrumentation as correctly as possible. We have developed extensive test suites to ensure that the code is correctly implemented.

In Paper I and Paper II, we have performed Experiments. Experiments (or controlled experiments) are, according to Wohlin, Runeson, Host, Ohlsson, Regnell, and Wesslen [23]

"an empirical inquiry that manipulates one factor or variable of the studied setting. Based in randomization, different treatments are applied to or by different subjects, while keeping other variables constant, and measuring the effects on outcome variables."

In Paper I and Paper II, we collected real-world data from our cases which were two large-scale software development companies. We used the data we had gathered to examine the thesis that machine learning can be used to automatically triage bugs. Specifically, we examined the thesis that using a special machine learning approach, called ensemble techniques, gives prediction accuracy that is at least as good as, or better than, a single specific machine learning technique.

In Papers III, IV and VI, we used mathematics to develop theories in the area of probabilistic text modeling and document classification. This was guided by the exploratory work in Papers I and II, where we found a possible avenue for improving the unstructured text handling in bug reports. To achieve this, we needed a fast and exact approach to modeling of text. We used computer science, mathematical and statistical, theories to develop a new, mathematically correct, and very fast parallel sampler for the LDA model.

Finally, in Papers V and VI we again used experiments in combination with quantitative data to determine whether or not our new approach showed the desired improvements.

Table 3.1 summarizes the main research methods and the purpose of the papers.

\begin{tabular}{lll}
\hline Paper & Method & Purpose \\
\hline Paper I & Empirical & Exploratory \\
Paper II & Empirical & Exploratory + Improving \\
Paper III & Analytical & Improving \\
Paper IV & Analytical & Improving \\
Paper V & Empirical & Improving + Explanatory \\
Paper VI & Analytical & Improving \\
\hline
\end{tabular}

Table 3.1: Mapping of each paper to research method and purpose. 
Again, although Paper II is listed in Table 3.1 as Improving, the work on that paper generated ideas that lead to the following papers.

Furthermore, we can break down the paper classification using the GQM approach by Basili [25]. Table 3.2 summarizes the main goal, and main metric used to evaluate the approach, and maps it to the research questions. In the sections below, the details of the individual papers are described.

\begin{tabular}{llll}
\hline Paper & Goal & Question & Metric \\
\hline Paper I & Feasibility of ML & RQ1 & Prediction Accuracy \\
Paper II & Feasibility of ML & RQ2 & Prediction Accuracy \\
Paper III & Exact Parallelism & RQ3 & Speed + Correctness \\
Paper IV & Rich Classifier & RQ3 & Accuracy + Richness \\
Paper V & Flexible Classification & RQ2 & Accuracy + Richness \\
Paper VI & Speed Improvements & RQ3 & Speed + Correctness \\
\hline
\end{tabular}

Table 3.2: Mapping of paper to research method, goals, and main evaluation metric.

\section{$3.4 \quad$ Paper I}

In Paper I, we collect roughly one year of real-world data from one large and complex system at Ericsson AB. We use minimal filtering of the data to ensure that the data is truly the real data that the engineers are working with, and that we get the maximum amount of data to work with. The only filtering we apply is to select solved bug reports (i.e finished) and exclude duplicates.

What characterizes the system is that it is mature and has been in production for over 10 years. It is a very large system and many different programming languages are used in its construction. The bulk of the code is implemented using $\mathrm{C}++$ and Java.

This setting was selected because it is typical for the context in which we propose that ML techniques can be used. The system is so large that, in practice, no single individual developer has detailed knowledge of the whole system. Assigning bug reports and locating the faulty component(s) in these types of systems is really challenging.

The main goal of the paper was to explore the feasibility of using ML techniques for AFL and ABA in large-scale software development projects in an industry setting. The paper mainly answers RQ1. The metric used for evaluation is prediction accuracy as measured by cross-fold validation. The main reason for using this measure is that cross-fold validation is a standard metric in the ML community and accuracy is a clear and simple metric to communicate to the industry stakeholders and is easy for them to understand. 


\subsection{Paper II}

Paper II is an extension to Paper I, where we further explore the effects and consequences of using machine learning for ABA in an industrial setting. The scope of Paper II is vastly increased compared to Paper I, both in terms of the number of cases and the evaluation. In Paper II, we collect more than 50,000 industry bug reports from two big companies and five different projects.

The goal of Paper II is to further study RQ1 and improve on the method of selecting ML classifiers, and to suggest guidelines for industry on how to deploy ML techniques for ABA and AFL in an organization.

Conclusions from Paper II guided the research of Paper III and Paper IV. One concrete question that is related to RQ2, and which suggests future work, concerns how to handle unstructured input, such as text. In the paper, we examine the performance of classification, both including and excluding text. We find that the combination of both text and structured data is the best. In the conclusion and future work section of Paper II, we propose research into the question of whether or not there are better ways to represent the textual input to the classifiers. This leads to papers III and IV, where we study techniques for representing unstructured text and using it for classification. The metric used for evaluation is again prediction accuracy as measured by cross validation.

\subsection{Paper III}

In Paper III, we mathematically derive a formulation of a probabilistic sampler for LDA and provide formal proofs for the complexity bound of the sampler. We then implement this sampler, and the comparison samplers, in code and study its characteristics compared to other approaches. In the paper, we prove mathematically that, although our sampler incurs an extra sampling step, complexity-wise, it is equivalent to competing implementations.

We also show empirically that the actual runtime behavior is favorable compared to other approaches that are approximate LDA models. The main method used in this paper is analytical, but the results of the sampler are evaluated empirically on different datasets. The datasets are the same datasets that are used in related papers using other implementations of LDA to facilitate close replication.

The goal was to develop a fast and correct method for representing text in ML techniques. It is related to RQ3 since it tries to address how we can improve on the techniques available in our research context. The metrics for evaluating the sampler are proofs of correctness and speed of the sampler. 


\subsection{Paper IV}

Paper IV is a further theoretical extension of Paper III, where we extend the unsupervised LDA sampler to a supervised classification model and study its characteristics. Again, the main method is analytical with an empirical evaluation on public datasets. The derived sampler is a Bayesian classifier, which implies that we get a measure of the uncertainty for all of the parameters in the model, including the predictions. This is very powerful since it allows an organization to tune the use of ML by trading prediction accuracy against automation level if desired. For example, when the uncertainty in predictions are low, automated intervention (routing of the bug report) can be used, but when the uncertainty is high, human intervention can be invoked.

The goal here was to implement a classifier that fulfills the requirements of handling both unstructured text and nominal values, as well as providing full probability distributions on parameter estimates. An additional goal was to implement a classifier that gives interpretable output. It relates to RQ2 in that it answers which techniques should be used in our context. But it is even more relevant to RQ3, since it improves on the techniques available. The metric used for evaluation was prediction accuracy using cross validation.

\subsection{Paper V}

In Paper V we take the experiences from Paper III and Paper IV and apply them to our original problem of AFL. We show that we get competitive results with our sampler from Paper IV. In addition, we get the richness of the Bayesian model, which gives several benefits. We exemplify one of these benefits by explaining how the richness of the model can be used in an industrial setting. For comparison, we use some of the same datasets that we used in Paper II. We complement these with publicly available datasets to facilitate replication.

The goal was to empirically evaluate how well the DOLDA classifier fulfills our requirements in our bug handling context. It answers RQ2 sine it deals with which techniques should be used in our context. The metrics used for evaluation were accuracy using cross validation and subjective measures of classifier richness.

\subsection{Paper VI}

In Paper VI we further improve on the LDA sampler described in Paper III.

The goal was to reduce the memory requirements and sampling time of the LDA sampler developed in Paper III, but still keep the exactness of the inference. These goals came primarily from the continued need to improve 
the sampling speed, but also from initiatives to implement LDA on Graphical Processing Units (GPUs).

Paper VI mainly relates to RQ3 since it improves upon machine learning techniques, both in general, and specific for our context. The metrics used to evaluate the goal was sampling speed and analytical proofs.

\section{$3.10 \quad$ Validity}

We discuss the various aspects of the validity of the research in the four forms detailed in the framework by Wohlin, Runeson, Host, Ohlsson, Regnell, and Wesslen [23]:

- Conclusion validity and Reliability - Conclusion validity is concerned with the relationship between the treatment and the outcome in a quantitative analysis. We want to make sure that there is a statistical relationship, i.e. with a given significance. Reliability is its counterpart in a qualitative analysis. This aspect is concerned with the extent to which the data and the analysis are dependent on the specific researchers. Hypothetically, if another researcher conducted the same study at a later time, the result should be the same. Threats to this aspect of validity are, for example, if it is not clear how to code collected data or if questionnaires or interview questions are unclear.

- Internal validity - If a relationship is observed between the treatment and the outcome, we must make sure that it is a causal relationship, and that it is not a result of a factor over which we have no control or have not measured. In other words, that the treatment causes the outcome (the effect).

- Construct validity - This type of validity is concerned with the relation between theory and observation. If the relationship between cause and effect is causal, we must ensure two things: (1) that the treatment reflects the construct of the cause well and (2) that the outcome reflects the construct of the effect well.

- External validity - External validity is concerned with generalization. If there is a causal relationship between the construct of the cause, and the effect, can the result of the study be generalized outside the scope of our study? Is there a relationship between the treatment and the outcome?

Often there is a trade-off between the different types of validity, and the various aspects of validity must be prioritized (Wohlin, Runeson, Host, Ohlsson, Regnell, and Wesslen [23, p. 111]). While all aspects of validity are 
important, our focus has been on solving real world large-scale industry problems as experienced by the author during many years of active development in the industry. Thus, our focus has been on External and Construct validity, i.e. that the research questions are relevant and that the experiments, as much as possible, use actual data that practitioners are working with. In our case, this has come at the expense of replicability on some occasions, since we are not able to make our datasets publicly available. This has been mitigated by using open datasets for comparison with the industrial data in Paper V, where open data was available that was similar to the industry data (unlike Paper I and Paper II where no similar data was available). Our studies are designed to minimize the impact of threats to validity. Below we discuss in detail the choices and decisions we have made in regard to these aspects.

\section{External Validity}

External validity concerns how well a study generalizes.

Effect of the threat on our results: What would the effect on our results be if they were affected by the threat? This is mostly related to Papers I, II and V, since Papers III, IV and VI are theoretical papers. If our results were not externally valid, this would mean that ML-based classification of bug reports would not be applicable to other large-scale industry software development contexts.

Large scale industry applicability is one of the main drivers of this work and a great deal of effort has gone into ensuring that the techniques are applicable to actual industry practice. One example of this is that in Paper II we examined a particular ML technique called Stacked Generalization (SG). The advantage of SG is that it is flexible and does not require particular expertise in deployment, due to its robust performance. The downside of SG is its lack of interpretability. This feedback from our industry partner (also backed by research [26]) lead us to explore richer techniques for dealing with unstructured text (Papers III \& VI) and Bayesian models for ML (Papers IV \& V).

We have gone to great lengths, exploiting the author's expertise and discussing with our industry partner, to ensure that the industry data that we have used is real world data from active projects, dealing with exactly the problems that the thesis deals with. The context of industry bug data is prohibitive, since this is very sensitive data and companies do not publish this type of data for many reasons.

For the main paper studying the applicability of ML-assisted ABA (Paper II) in an industrial context, we managed to gather data from five major industry projects, from two large software development companies. This is the largest study on industry data that we are aware of. For replicability and comparison with Open Source Software (OSS) data, we have also applied the techniques to OSS datasets. Two companies is not a big sample of large- 
scale software development companies, but we argue that the structure and setup of the bug handling and teams generalizes to many types of large-scale software development contexts. Additionally, our results are in line with previous research that has been done, both in an OSS context, and in smaller industrial research papers [27]. The threat to external validity is further mitigated by the fact that no project-specific tuning of the classifiers has been done, nor have we filtered the bug reports to achieve greater accuracy. We argue that our accuracy results are probably on the lower end of what can be achieved, since no real optimization of classifiers or advanced feature selection was performed.

Papers III, IV and VI are theoretical papers and the question of external validity is simpler to answer. In this case we need to show that the samplers fulfill the defined requirements. This is shown analytically and measured using standard datasets.

Paper V is specifically targeted to mitigate some of the problems of applicability to industrial context of Paper II (i.e. external validity). Here we argue that the richer model that Paper V presents complements the results in Paper II and the richer model further increases the applicability to the industrial context.

\section{Conclusion Validity}

Many aspects of the conclusion validity threat deal with statistical power and violations of the assumptions of statistical tests.

Effect of the threat on our results: What would be the effect on our results if they were affected by the threat? Statistical conclusions about measures would not hold.

One threat to conclusion validity is the use of cross validation to draw conclusions about the generalization error of classifiers in this thesis. The reason we use cross validation (in spite of known problems [28] with it) is that it is a standard method that researchers and the statistical and machine learning communities are very familiar with and it is simple to understand for our stakeholders. In Paper II we mitigate this problem by exploring other methods than cross validation, and we conclude with a warning that cross validation should not be the only type of evaluation. So one needs to be careful drawing absolute conclusions from cross validation alone. Mostly, we use cross validation as a model comparison tool, to compare how models and behaviors differ. For this purpose, cross validation should suffice since all models (in our context) should be equally afflicted by the limitations of cross validation. We discuss these aspects quite extensively in the validity section in Paper II. Another aspect that limits our exposure the conclusion threats are the amount of data that we have gathered to ensure minimum exposure to problems with statistical power. 
Other aspects of conclusion validity concern the reliability of treatment implementation and irrelevance in the experimental setting. Since we are not performing any experiments or studies on human subjects, only in-silico, that is, only on computer models, we are not affected by aspects that involve human interaction. We have implemented extensive test suites to ensure that our code is behaving correctly.

Reliability of measures is another aspect of conclusion validity. In our case this translates to measurements based on bug reports. Fortunately, the tools that deal with bug databases tend to be tools that are used on a daily basis and are therefore quite well tested and robust in terms of measurements. Another aspect of measurement that is worth noting is the interpretation of derived measures from bug databases. For instance, in a complex bug flow, when should we say that a bug has reached a specific team? This is not always trivial to decide. Also, when should we conclude that a bug is solved? In the industrial bug flows that we have studied, the flows are typically much more complex than in Open Source systems, such as Bugzilla. Here the author has used his comprehensive experience of working with these systems to define reasonable measures for these aspects, and they have been discussed with the teams and persons responsible for the bug handling process.

\section{Internal Validity}

Internal validity deals mainly with how confounding variables can affect causal relationships drawn from statistical results.

Effect of the threat on our results: What would be the effect on our results if they were affected by the threat? The ensemble combinations in Paper II would look different, but it is unlikely that this would substantially affect our results. Since our primary contribution is the suggested methodology rather than the specific classifiers, we argue that this is unlikely to affect our results.

Since we are not making many strong causal claims based on statistical results, internal validity is not a major threat to this thesis. For instance, we don't claim that a particular variable in a bug report causes a bug to be located in a particular subsystem. In Paper II, one threat to internal validity is that we do not optimize the individual classifiers in the ensembles. This could cause some classifier with better default settings to be favored. Since we do not make strong claims on exactly which classifiers to select, but rather suggest a procedure for selecting the classifiers, we argue that this limits the threat to validity. When it comes to the accuracy measures, individual tuning of classifiers should only improve accuracy results. 


\section{Construct Validity and Reliability}

Construct validity concerns the relationship between the background theory and how it connects to the actual experiments and measurements that the researcher performs in a study.

Effect of the threat on our results: What would the effect on our results be if they were affected by the threat? This would mean that other researchers would reach conclusions that were different from ours.

Papers I, II, and V could arguably be susceptible to threats to construct validity. We argue that this threat is limited due to the many years of work experience of the researcher, whose daily work involved dealing with the issues that these papers confront. Reliability concerns the threat that other researchers would not reach the same conclusions due to procedures in data collection and analysis. For Papers I and II, it is harder since we cannot publish our datasets and a direct comparison is difficult. To increase reliability, we have described in detail in the individual papers how data was collected and the context of the studies. For Paper V, we complement the industry data with OSS data to facilitate replication. The source code is also published openly to increase transparency.

It is possible that other researchers could reach different conclusions regarding the use of stacking as a primary ML method for AFL and ABA as proposed in Papers I and II. Our choice here was mainly guided by a desire for simple deployment in a large-scale software development organization where the goal was to maximize accuracy despite limited ML expertise. Other researchers in different contexts with other priorities could reach other conclusions regarding the best option in terms of classification techniques. For Paper V, where model richness and interpretability were the main goals, it is also possible that other researchers would reach other conclusions since there is a certain amount of interpretation with regard to what richness and interpretability means. Although details may differ, we argue that we have presented enough evidence for our main point, that $\mathrm{ML}$ is a feasible option for the ABA and AFL problems in an industrial setting.

Papers III, IV and VI should not be affected by these threats since they are mainly analytical and the context and requirements are very clear. For Papers III, IV and VI, it should be straightforward for other researchers to replicate our results since we use datasets that are research community standards for evaluating LDA samplers and classifiers. 


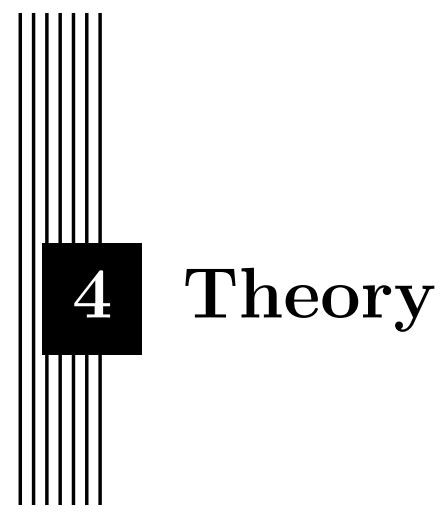

The study of machine learning has grown to be a vast field and there is no room (or time) to cover all of the nuances and aspects of the field in this thesis. This chapter is mainly intended for the unfamiliar reader or the industry practitioner with some, perhaps old and slightly faded, familiarity with basic probability theory, calculus and linear algebra. In the exposition below we have tried to minimize the use of mathematical notation that might be confusing or that could obscure the intuition of what is happening. The hope is that this chapter will give a basic understanding of some the core mathematical ideas behind a few of the most important machine learning techniques used in this thesis. Hopefully, this will enhance the reading and understanding of the papers that make up the thesis. For more thorough expositions we refer to the excellent works of Bishop [29] and Murphy [30].

Personally, I have found that nothing facilitates learning as much as connecting the math to simple examples. So the goal is, as much as possible, to exemplify the math with examples from our problem domain of bug reports. The hope is to build up an intuition of the area and the theory that we will be discussing in the included papers from a basic level. Hopefully this will give the unfamiliar reader some of the basic understanding that is needed to read the rest of this thesis. 


\subsection{Machine Learning Introduction}

To start getting an intuition of what machine learning is, we discuss various flavors of learning and ways of implementing the learning approaches. First, we start with some basic definitions and examples.

- Classification

- Classifying data into a set of discrete outcomes

- Example: Which software component does a bug report originate from?

- Regression

- Estimate of a real value from data

- Example: What will the processor load be if we activate a set of features in the system?

- Clustering

- Finding groupings in unlabeled data

- Example: Which types of users behave the same way in a telecom network?

- Dimensionality Reduction

- Extract the most important piece of information from a large set of data

- Example: Given a text document with 1000 words, which are the most important ones?

- Visualization

- Displaying information in a way that a humans can more easily study a dataset

- Communicating results of a study in overview format

- Example: See Figure 4.1

\subsection{Probability Reminder}

This section provides a very short reminder of some of the basic concepts in probability theory. This section can safely be skipped if the reader is familiar with the nomenclature and basics of probability theory. For a very readable introduction to probability we highly recommend the beginning chapters of Bishop [29]. 


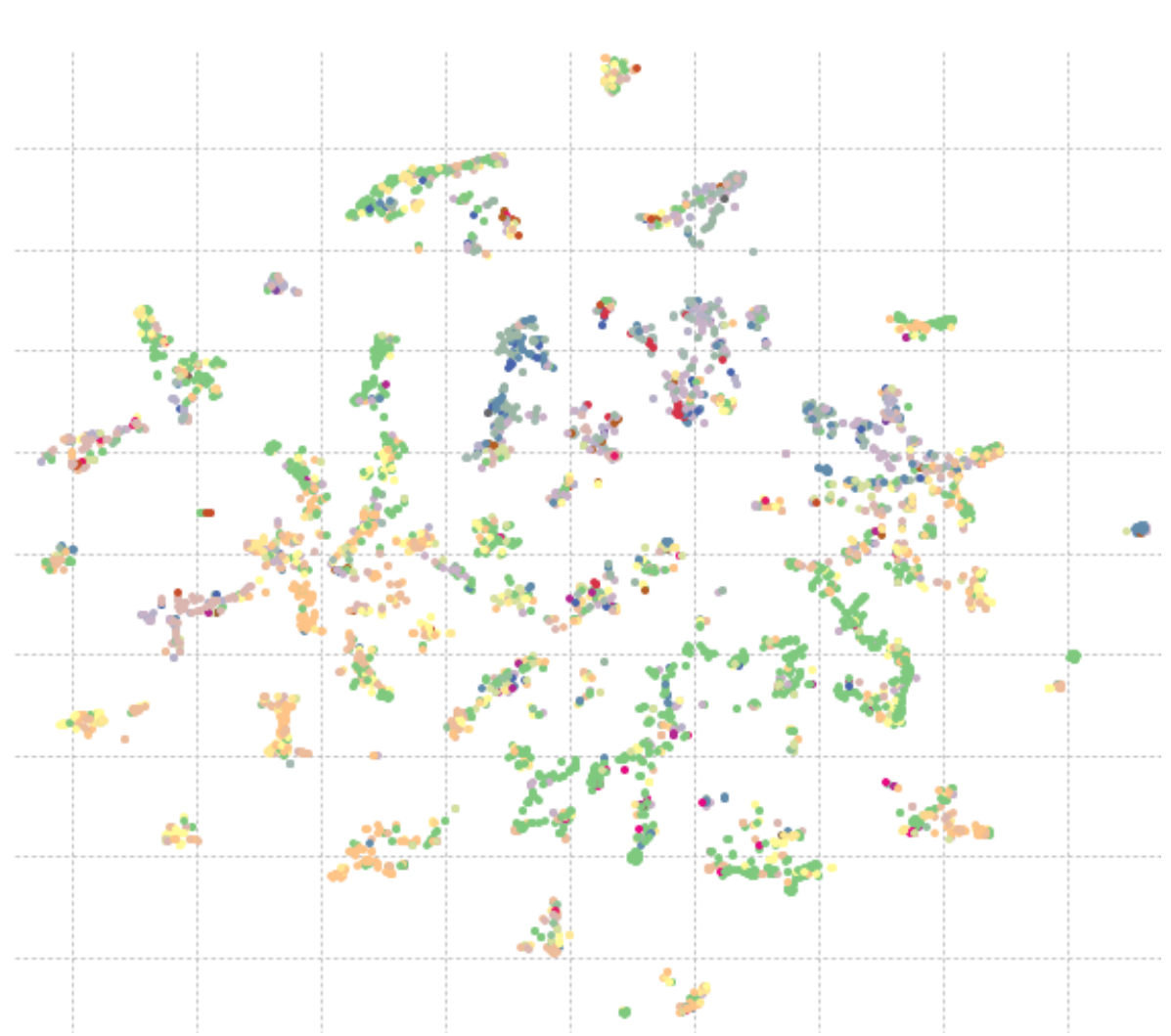

Figure 4.1: t-SNE rendering of a bug database using unstructured text via LDA, using the individual document topic mixture as input features.

The probability of an event $A$ occurring is usually written as

$$
p(A)
$$

Probabilities range from 0 , indicating an impossible event, to 1 , which is a completely certain event. Frequently, probabilities are expressed in percentages, ranging from 0 to $100 \%$.

Discussing probability only in terms of events fast becomes burdensome so we quickly introduce random variables (RV). A random variable is a function that takes outcomes of events as inputs and maps them to numbers. For instance, a random variable $\mathrm{X}$ could take the event "the rolled die landed with 4 dots up" and map that to 4 . Another RV Y, could represent the sum of two die roll events, returning 7 if one die landed on 4 and the other on 3 . Formally, the definition of a random variable is [31]

Given an experiment with sample space $\mathrm{S}$, a random variable (RV) is a function from the sample space $\mathrm{S}$ to the real numbers. 


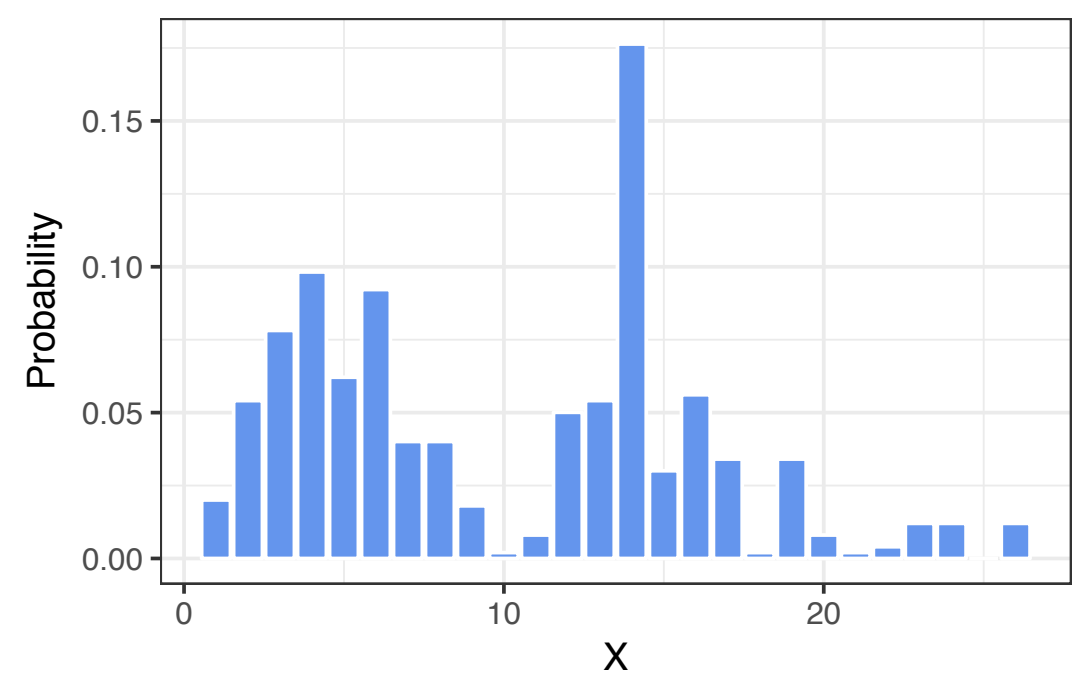

Figure 4.2: Discrete probability distribution.

There are some subtle points about notation to be made here. Probabilities are only defined on events, but not on RV's, so strictly it does not make sense to talk about $p(X)$ if $X$ is an RV [31]. But we can talk about $p(x)$ (note: small letter $x$ ), where $p(x)=p(X=x)$, since having $X$ take the value $x$ is an event, this is fine (we assume that $X$ is discrete). We can also talk about $p(5<X<8)$ (valid for both discrete and continuous RV's) which is the event that the RV X lies in the 5-8 interval. This distinction is not always clear (especially in less formal contexts) and the notation $p(X)$ is used when it is understood that it stands for $p(X=x)$ (see Bishop [32]). We will also use this notational shortcut to make the presentation simpler, but with the warning that sometimes it can be confusing.

A distinction is made between discrete and continuous random variables. A discrete RV maps outcomes to a discrete list of outcomes. The list can be finite, as in a fixed set of outcomes (the six sides of a die), or infinite (the integers). A continuous RV maps outcomes to the real numbers. Sometimes the notation of discrete and continuous RV's differ, with continuous probabilities written with lowercase $p$ and discrete ones with a capital $P$. We shall not bother with this distinction in this text and we will use lowercase $p$ for probabilities.

The probability of a random variable is completely described by its probability distribution, which is a function where the domain consists of all possible outcomes of the RV. As a consequence of the basic axioms of probability, the integral of a probability distribution from $-\infty$ to $\infty$ sums to 1 . A probability distribution can be either discrete as in Figure 4.2, or continuous as in Fig- 


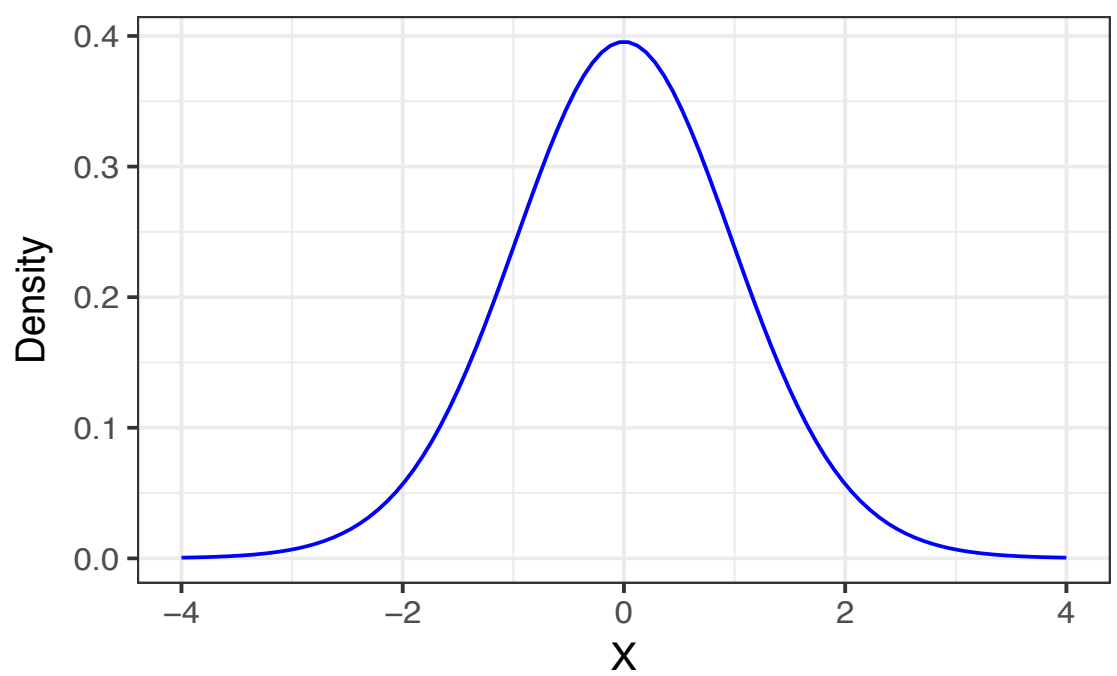

Figure 4.3: Continuous probability distribution.

ure 4.3. If it is discrete, it is called the probability mass function (pmf). From the probability mass function we can, for each possible value the random value can take, find its corresponding probability. For instance, from Figure 4.2 we can see that the probability of $x=12$ is ca $5 \%$, i.e. $p(X=12)=5 \%$.

The probability distribution is continuous if the random variable of interest is a real number. This is depicted in Figure 4.3. In this case the distribution is called probability density function (pdf).

There is an important difference between a discrete distribution and a continuous distribution. In the continuous case we are interested in the probability of a real value, but the probability of any specific real-valued outcome of a continuous $R V$ is 0 ! So, in contrast to the discrete case where we can look at the distribution plot (pmf) and read off the probability of the RV for a specific outcome, we cannot do this from the pdf. It is entirely possible for the density (the y value of the pdf) to have values larger than 1 , which we know is impossible for probabilities, since they are defined to lie between 0 and 1. What we can do with the pdf is to find the probability that the outcome of a continuous RV lies within an interval. This is depicted in Figure 4.4, where the interval is $-1<X<1$ and the associated probability is $68 \%$. This probability is found by taking the definite integral of the pdf from -1 to 1 , i.e.

$$
p(-1 \leq X \leq 1)=\int_{-1}^{1} p d f(x) d x=68 \%
$$

Note here that $-1<X<1$ is the event that the RV $X$ lies in the interval. 
We can also talk about the probability of two or more simultaneous events. The probability of both events $A$ and $B$, called the joint probability of $A$ and $B$, is written

$$
p(A, B)
$$

An example of this would be the toss of a coin and a roll of a die. Where $A$ could be the event "the coin landed Heads up" and $B$ is the event "the die landed with an even number up". The joint probability is the probability of both those two outcomes occurring in the same trial. Joint probabilities generalizes to RV's also, where we talk about the joint distributions of two or more RV's.

From a joint probability distribution, we can margnialize out or integrate out one variable and get the marginal distribution for the other. In Section 4.76 we show a detailed example of marginalization in a concrete case. Marginalization is slightly different depending on whether the RV is discrete, i.e., its outcomes can be mapped to the natural numbers, or continuous, in which case its outcome is a real number. The marginalization of a continuous $\mathrm{RV}$ is done via integration.

$$
p(X)=\int_{Y} p(X, Y) d Y
$$

while for a discrete RV it is the sum

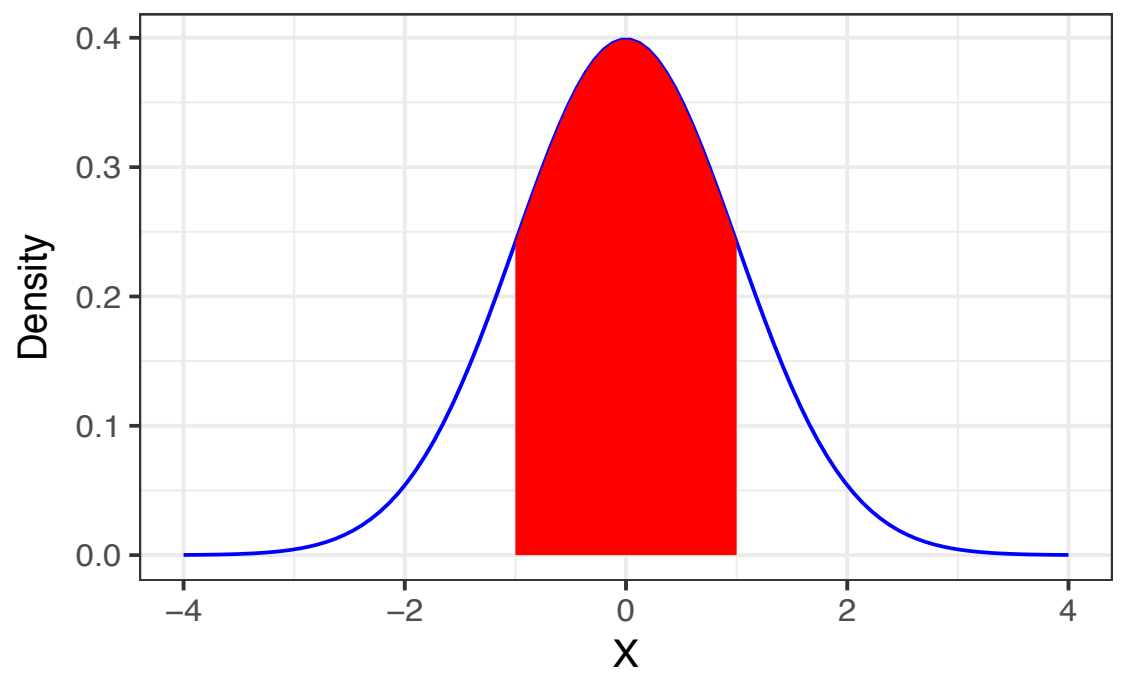

Figure 4.4: The probability of a continuous probability distribution is the area under the curve of the continuous probability distribution, not the y value of the pdf! 


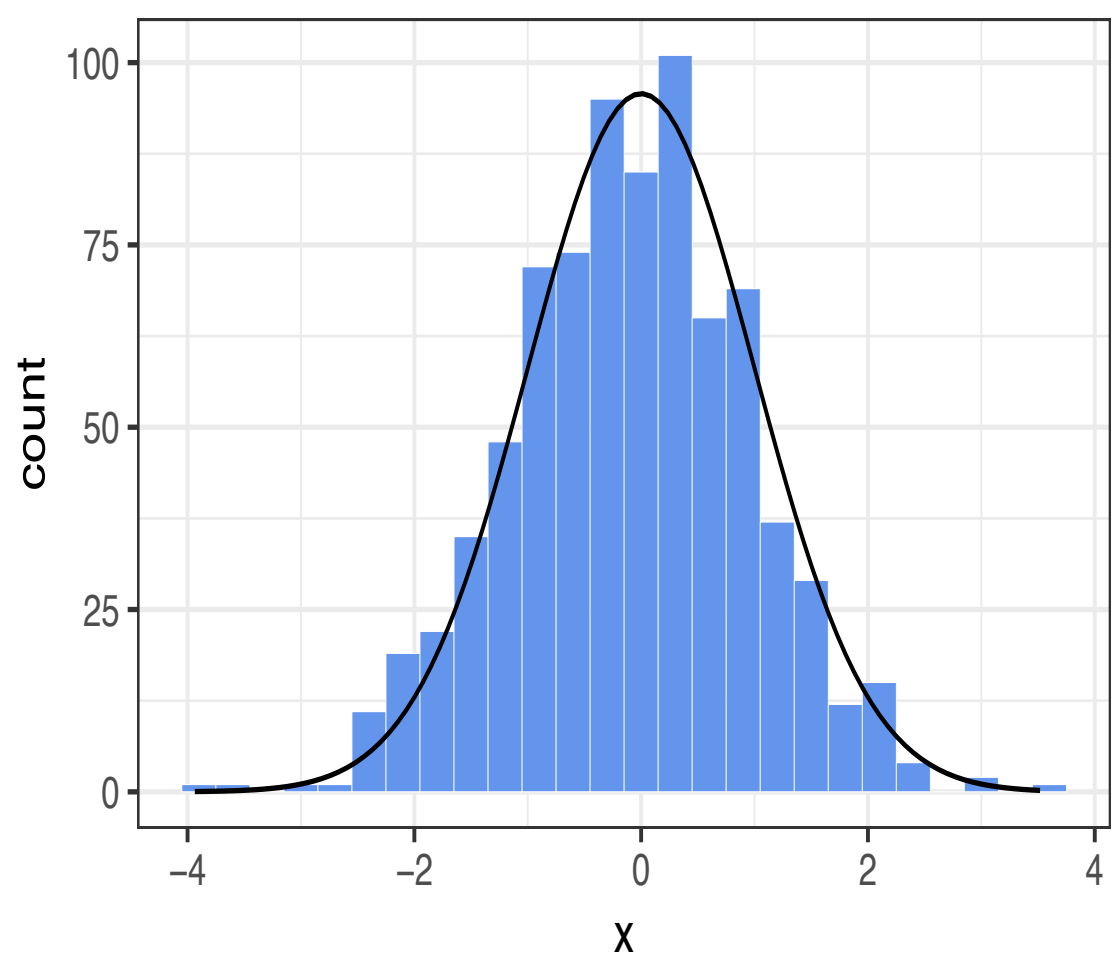

Figure 4.5: Histogram of 800 draws from a normal distribution with the true normal overlayed.

$$
p(X)=\sum_{Y} p(X, Y)
$$

A distribution is not only the graphical view of a random variable, it is also a mathematical object. We have already mentioned that we can plot the distribution in a graph, as in Figures 4.2 and 4.3, and that we can use the distribution to calculate the probability of the outcomes of a random variable. Another thing we can do is to draw samples from it. When we draw samples from a distribution we basically tell the distribution to generate a random outcome of the RV proportional to its probability according to the distribution. So, for instance, if we have an RV that has a normal distribution, we can tell it to generate 800 samples from its distribution. In Figure 4.5 we have done just that and plotted the samples in histogram form with the true normal pdf overlayed.

We can also talk about conditional probability. Conditional probability is the probability of an RV conditioned on some knowledge. This is written 
$p(A \mid B)$ and should be read as the probability of $A$ given $B$ or the probability of $A$ conditioned on $B$. Conditional probability is defined as:

$$
p(A \mid B)=\frac{p(A, B)}{p(B)}
$$

An example of this would be, the probability of a bug being located in a particular subsystem (say the UI) conditioned on the fact that we know that the bug was reported by a specific customer. This is written as $p($ Component $=U I \mid$ Customer $=$ AcmeTelco $)$. We can then calculate this according to Equation 4.1.

$$
\begin{aligned}
& p(\text { Component }=U I \mid \text { Customer }=\text { AcmeTelco }) \\
& =\frac{p(\text { Component }=U I, \text { Customer }=\text { AcmeTelco })}{p(\text { Customer }=\text { AcmeTelco })}
\end{aligned}
$$

Intuitively this can be thought of as follows. Say that we have a database with bug reports. We then count how many bug reports were reported by the customer AcmeTelco (denominator in Equation 4.2). We then count how many of the AcmeTelco bug reports were reported on the UI component (nominator in Equation 4.2). By dividing these two numbers we get $p($ Component $=U I \mid$ Customer $=$ AcmeTelco $)$.

We must also briefly mention the extremely important Bayes Rule, which we will discuss further in Section 4.4. Bayes rule states that

$$
p(A \mid B)=\frac{p(B \mid A) p(A)}{p(B)}
$$

and follows directly (see Section 4.4 for a derivation) from conditional and joint probability. Bayes rule is often used in its proportional form when we are not directly interested in the specific probabilities, but rather in comparing the sizes of the probabilities to make a decision.

$$
p(A \mid B) \propto p(B \mid A) p(A)
$$

The $\propto$ (read "proportional to") means that $p(A \mid B)$ is not a normalized probability distribution (i.e. it does not sum to 1 ), but can be made so by multiplying it by an expression that is not dependent on $A$.

An example of this is when we want to make a classification and we are only interested in selecting the class with the highest probability. Equation 4.4 lets us do this, since the denominator in Equation 4.3 will be the same for all classes, so we can ignore the denominator and just pick the class that has the highest value of $p(B \mid A) p(A)$. This is because if the proportional form has the highest probability of a class, then the normalized expression will also have the highest probability for the same class. The proportional form of Bayes 
rule is also used in so called Markov Chain Monte Carlo (MCMC) sampling. In MCMC we often do not need to know the exact probabilities, just the shape of the probability distribution.

\subsection{What is Learning?}

Before we discuss more details of machine learning, we can try to define what learning is. Tom Michell, one of the pioneers in the machine learning field, defines learning (in the context of ML) in the following way [33]:

Definition 1. "We say that a machine learns with respect to a particular task $T$, performance metric $P$, and type of experience $E$, if the system reliably improves its performance $P$ at task T, following experience $E$ "

While slightly cryptic, it is a succinct description of what, in our context, it means to learn. To make the definition and the following chapter more concrete we will use a running example to accompany the text to hopefully clarify the sometimes obscure meaning of the math and theory. We start the example here by setting the task $T$ of the ML system to be that of predicting which component in a software system that a bug is located in. The performance metric $P$ will be the prediction accuracy the ML system achieves, that is, how many of the bugs it correctly predicts divided by the total number of predictions it has performed.

$$
\text { prediction accuracy }=\frac{\# \text { correct predictions }}{\# \text { total predictions }}
$$

Defining $E$, the experience is the most elusive task. A major part of formalizing experience consists of defining how we encode the experience so we can talk about it formally. This will be discussed extensively in this thesis; for now we will simply state that gaining more experience consists of "seeing" more bug reports.

Now that we have all the major constituent parts of our definition, let's try it out. We say that our ML-system learns, if it gets better at predicting which software component contains the fault described by a given new bug report, the more historical bug reports that we "show" it. An erroneous, implicit assumption that is often made by readers unfamiliar with the statistics and machine learning fields, is that there is a linear relationship between the amount of experience that is shown to the system and the amount that the system learns. I.e. the assumption is that the more data you present to the ML-system, the more it learns. This is not generally so. As we show in the bug assignment context in Paper II [34], if you show the ML-system too much, especially too old, bug reports it actually gets worse at predicting new bug reports. This is intuitive, since very old bug reports is probably not representative of the bugs that are currently generated. This is because the 
software has evolved and developers are working on other parts of the system and other features than was worked on a long time ago. The old bug reports were in the old components of the system while new bug reports tend to be on the newly updated parts of the system.

Another important aspect of how much a system can learn is the quality of the input data. If the data presented to the ML-system does not contain enough relevant information to learn from, there is, per definition, a limit to how much can be learned. The aspect of data quality can be split into two parts. One part concerns how well the data is collected and pre-processed before it is presented to the ML-system, this is often called feature engineering. The other part deals with the natural variation or noise in the data and systematic errors that can be part of the data collection process. There is a limit to how accurate the system can maximally become depending on this natural variation in the data. To get some intuition for this problem, imagine that the task of a prediction system is to predict whether a person is male or female based on only the length of the person. Since men tend to be taller than women a general strategy would be to guess male if the person is relatively tall and female if the given length is shorter. Due to natural variation in length in both men and women there is a limit to how accurate this prediction will be, since some men are shorter than average and some women are taller than average. ML techniques are by their nature imprecise, since if there was an exact way to solve the problem one would not need to resort to ML techniques. The exception to this rule is when the exact techniques are computationally too demanding but theoretically possible, but we will ignore these cases here. Quantifying the magnitude of the imprecision is an important aspect of ML techniques. Sometimes it is possible to quantify the imprecision but sometimes not, it depends on the particular ML technique used.

\section{Data quality}

The general rule is that the better data you give an ML-system the better output predictions you get from the system. This is in no way specific to machines, but applies equally to humans. If you teach humans using knowledge from the 17:th century (old data, bad data), humans also become worse at understanding their environment. There is no inherent mechanism in learning that prevents adverse learning, rather, protecting against it must be encoded in the learning algorithm. An example of protection against adverse learning could in this case be to weigh old bug reports less than newer ones, so they have less influence in what the ML-system learns. Concretely this could be done by looking at the filing date of the bug report and scale the importance of the bug report proportionally to its age, i.e. how many days old it is. The decreasing weight of older data is often called weight decay. 
Finding, or learning, or inferring, the exact weights used to scale different components (in this case the age) of the data (the bug reports) turns out to be a crucial part of learning.

From now on when we talk about learning in this thesis, we always take that to mean finding an algorithm that can be executed by a computer, that fulfills the criteria in Definition 1. We make a clear distinction from learning in this specific context and the general concept of learning that applies to humans.

\section{Learning Using Linear Regression}

It is probably safe to say that linear regression or ordinary least squares (OLS) is the mother of all learning techniques. It was one of the first (developed by Sir Francis Galton and Karl Pearson) and is still one of the most important techniques in the field. Linear regression is not only very important in that it is a basic solution to a fundamental problem, but it also has a lot of nice properties. The main ones being that it is one of the simplest to understand and interpret and it is a crucial building block in the ML toolkit. As a building block, linear regression is the basis for many more advanced techniques that directly extends linear regression to other types of learning.

Linear regression solves the fundamental problem of going from observations of a real value given specific inputs, to inferring the parameters of a linear model.

We will start with the most basic linear equation. Here our response variable or dependent variable is a function of only one input variable or covariate or predictor $^{1}$ (variable) $x$ and a fixed parameter slope, it has the form:

$$
y=\text { slope } * x
$$

Equation 4.6 says that as $x$ increases, $y$ increases by slope times $x$. Another way to put it is that a one unit increase in $x$ increases $y$ by slope. This view is important since it is often how the linear model is used in practice.

If we know the value of the slope we can now calculate the corresponding value of $y$ given a new $x$. The problem, and the reason that we use machine learning, is that we usually do not have the slope, but we have observations of $x$ and the corresponding $y$ values. This can look something like Table 4.1. The data in Table 4.1 is simulated by picking values for the slope and the intercept and then drawing some random values for $x$, calculating it's corresponding $y$ and adding some random noise. The simulated data $y$ could for instance be the measured CPU load in percent on a node when $x$ number of users are connected to that node.

\footnotetext{
${ }^{1}$ sometimes predictor variables are said to be created as a function (which can be the identity function) of input variables
} 
Here we should immediately make clear that when modeling a percentage with linear regression we are violating some fundamental assumptions of linear regression. A percentage can only range between 0 and $100 \%$ but there is nothing inherent in linear regression that limits the domain to the $0-100$ range. Another example of an improper modeling would be to model a count, which has a lower limit of zero, and a domain of only integers. When sometimes these types of improper modelings are done, it is important to be aware that underlying assumptions are broken and the result should be carefully scrutinized to decide if it is reasonable.

The goal of linear regression is to find the value of the slope of a linear model so we can use it to predict the load on the machine given that a certain number of users are connected.

$$
\hat{y}=f(x)=\text { slope } * x
$$

In Equation 4.7 we use a fitted value of slope to predict a new $\hat{y}$ value given some $x$.

Finding the value of slope is called fitting the data to a model, or learning the model parameters. The resulting model could for instance be used in dimensioning guidelines to customers.

\begin{tabular}{rrr}
\hline & $\mathrm{y}$ & $\mathrm{x}$ \\
\hline 1 & 14 & 20 \\
2 & 17 & 120 \\
3 & 31 & 330 \\
4 & 33 & 340 \\
5 & 6 & 73 \\
6 & 23 & 310 \\
7 & 14 & 78 \\
8 & 36 & 360 \\
9 & 25 & 320 \\
10 & 33 & 200 \\
\hline
\end{tabular}

Table 4.1: 10 observations of the real-valued variable $y$ given inputs $x$

If we want to fit the data in Table 4.1 using a linear model how would we do that? The process of fitting data to a linear model means that we want to find the parameters (slope in this case) that describes a line that fits the data points as good as possible. In Figure 4.8 the data from Table 4.1 is plotted in a so-called scatter plot.

We can quantify the notion of as good as possible by saying that we want to minimize the total error we get if we use the model. So what is the error? The error for one data point could be defined as the distance between an actual $y$ value at a point $x$ and a point $\hat{y}$ that is predicted by the model by plugging in the $x$ value into the linear regression model, i.e. $y$ - $\hat{y}$. It turns out 


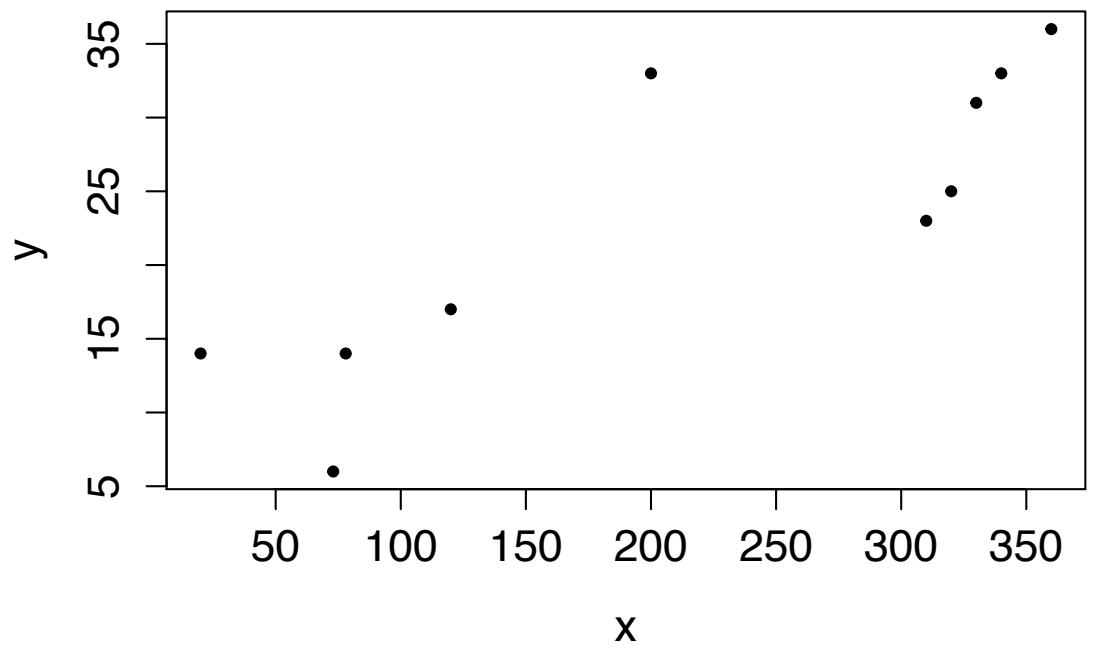

Figure 4.6: Scatter plot of data in Table 4.1.

that for several reasons it is even better to use the squared error, i.e. $(y-\hat{y})^{2}$. The squared error will punish large deviations from the predicted value more than small ones. We now have a definition of the (squared) error at one data point,

$$
e^{2}=(y-\hat{y})^{2}
$$

which we can also express as a function of our inputs $x$ :

$$
e^{2}=(y-(\text { slope } * x))^{2}
$$

but we want to minimize the total error of using the model for all $M$ points ( $M=10$ in our example) so we need to sum up the errors from all points. This quantity is called the sum of squared errors (SSE) and is expressed in Equation 4.10.

$$
\text { sse }=\sum_{i=1}^{M}\left(y_{i}-\hat{y}_{i}\right)^{2}
$$

If we plug in our linear expression $($ slope $* x)$ for $\hat{y}$ into Equation 4.10 we get.

$$
\text { sse }=\sum_{i=1}^{M}\left(y_{i}-\left(\text { slope } * x_{i}\right)\right)^{2}
$$


So now we have an expression for the total error of the model in terms of $y, x$ and slope. What does this give us? Well, it gives us a quadratic formula for the error as a function of $y, x$ and slope. We can now vary slope and calculate the corresponding error of the model. Figure 4.7 shows a quadratic function where the SSE is plotted as a function of slope.

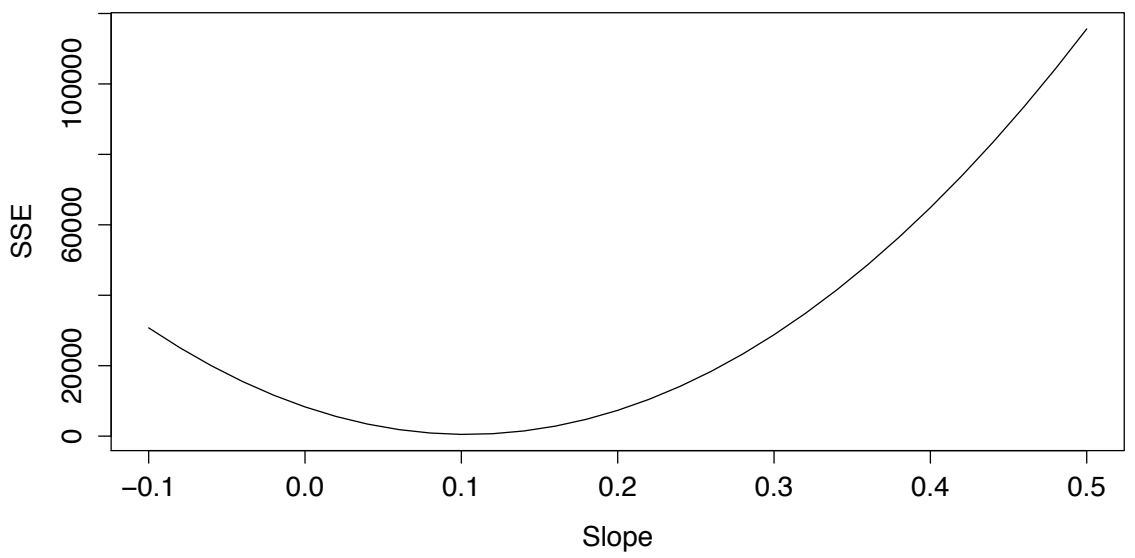

Figure 4.7: Plot of the sum of squares error (SSE) as a function of the slope. We see that there is one point where the error is at a minimum and we know that this is a global minimum since the error is a quadratic function. We know from calculus that the slope of the tangent (i.e. derivative) at the minimum of a quadratic function equals zero.

A quadratic function such as the SSE, is a special case of a class of functions that is called convex functions, and a defining property of (strictly) convex functions is that they have a global minima. This is convenient since we would like to find the value of the slope that minimizes the SSE. We remember from calculus that if we take the derivative of a function $f$ we get a new function $f^{\prime}$, that describes the rate of change in the original function $f$. To find the minima of the quadratic SSE, we take the derivative with respect to the slope and set this derivative to zero, we can then solve for slope to find the value of slope that gives us the minimum error.

We take the derivative of the SSE with respect to the slope by using the chain rule from calculus.

$$
s s e^{\prime}=\sum_{i=1}^{M} 2 *\left(y_{i}-\left(\text { slope } * x_{i}\right)\right) *-x_{i}
$$


We then set the derivative to 0 and solve for slope.

$$
\begin{gathered}
0=\sum_{i=1}^{M} 2 *\left(y_{i}-\left(\text { slope } * x_{i}\right)\right) *-x_{i} \\
0=\sum_{i=1}^{M}-2 *\left(y_{i}-\left(\text { slope } * x_{i}\right)\right) * x_{i} \\
0=\sum_{i=1}^{M}\left(y_{i}-\left(\text { slope } * x_{i}\right)\right) * x_{i} \\
0=\sum_{i=1}^{M}\left(y_{i} * x_{i}-\text { slope } * x_{i}^{2}\right)
\end{gathered}
$$

Distribute the sum and pull out constants in front of the summation.

$$
0=\sum_{i=1}^{M}\left(y_{i} * x_{i}\right)-\text { slope } * \sum_{i=1}^{M} x_{i}^{2}
$$

Solve for slope.

$$
\text { slope }=\frac{\sum_{i=1}^{M}\left(y_{i} * x_{i}\right)}{\sum_{i=1}^{M} x_{i}^{2}}
$$

This result can be re-written as:

$$
\text { slope }=\left(\sum_{i=1}^{M} x_{i} * x_{i}\right)^{-1} * \sum_{i=1}^{M}\left(x_{i} * y_{i}\right)
$$

If we use 4.19 together with the numbers from Table 4.1 to calculate a value of slope we get the result 0.095 .

We can then plug that value into our original formula:

$$
y=\text { slope } * x
$$

and use this to plot the purple line in Figure 4.8. We have just solved our first learning problem, we have learned the value of slope from data! We can quantify the quality of our learned parameter by what SSE it gives. In this case the SSE is 426. But can we do better? Now it is time to introduce the intercept into the model and see which effect it has on our results. With the approach we have taken above we have implicitly assumed that the intercept of the function is zero. With this assumption, we force the regression line to pass through zero, and this might not always be a good model. A more generic approach is letting the model learn the intercept also, i.e. the new model is formulated as:

$$
y=\text { intercept }+ \text { slope } * x
$$




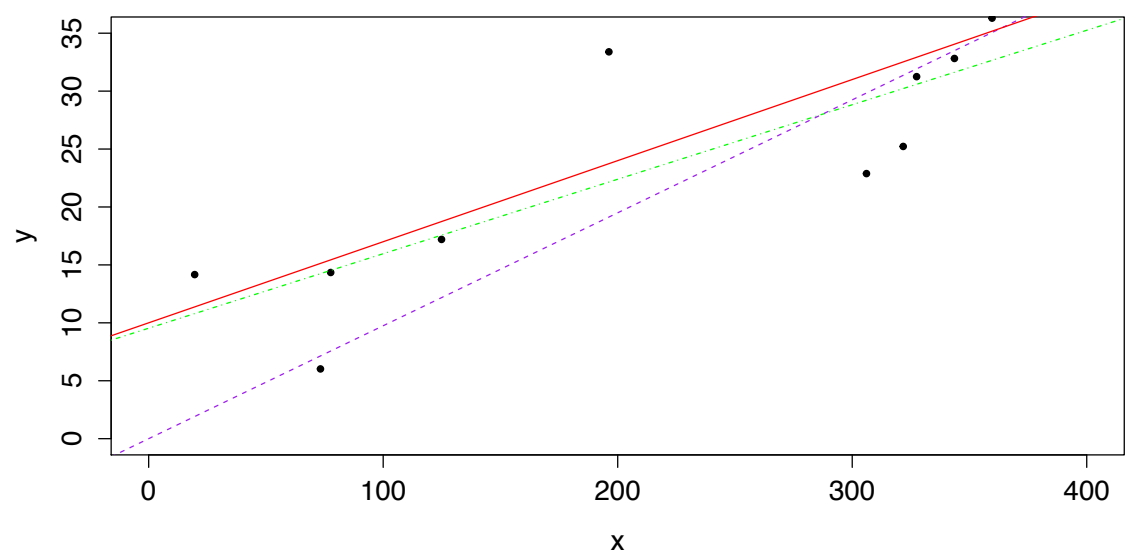

Figure 4.8: Plot of data in Table 4.1 with various fits. The solid red line is the "real line" that was used to simulate data from. The purple dotted line is the regression line of a single variable linear model without intercept, and the green dash-dotted line is the regression line of a model fitted with an intercept.

We may also have more input variables than just one $x$. We can then extend the model with more explanatory variables. If we have $N$ variables the model then looks like this:

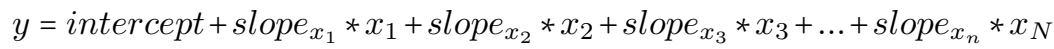

We can use the same reasoning and derivation as for the simple case above, but since we have several variables, we have to take partial derivatives with respect to each of the variables in the expression. Taking partial derivatives simply means taking the derivative of an expression with respect to each of the variables one at the time and holding the other variables constant. We will not do that derivation here, since we shall later see that if we formulate the problem as a linear algebra problem we get an elegant and equivalent solution to the problem. To achieve this, we do some notational updates to clarify the presentation. In the machine learning field it is common to use the letter $w$ (for weight) instead of using slope. With this notational update, the form of the linear model looks like this:

$$
y=\text { intercept }+w_{1} * x_{1}+w_{2} * x_{2}+w_{3} * x_{3}+\ldots+w_{n} * x_{N}
$$


To further simplify notation, the intercept can be called $w_{0}$ which gives us:

$$
y=w_{0}+w_{1} * x_{1}+w_{2} * x_{2}+w_{3} * x_{3}+\ldots+w_{n} * x_{N}
$$

This can be re-written using the summation symbol as follows:

$$
y=w_{0}+\sum_{i=1}^{N} w_{i} * x_{i}
$$

This is implicitly:

$$
y=w_{0} * 1+\sum_{i=1}^{N} w_{i} * x_{i}
$$

I.e we can add a "dummy" $x_{0}$ which is always 1 which represents the intercept (if we want an intercept, which in general we do when we solve general regression problems). If we then add this "dummy" $x_{0}$ and require that it is always set to 1 we can express our equation for one $y$ like this:

$$
y=\sum_{i=0}^{N} w_{i} * x_{i}
$$

This is an expression for one y, if we have several $y$ 's we index them using $m$. The equation for one $\mathrm{y}$ is then:

$$
y_{m}=\sum_{i=0}^{N} w_{i} * x_{m i}
$$

If we have $\mathrm{M}$ rows with $\mathrm{N}$ covariates we can make even more notational simplifications and express this as a linear system where bold font indicates that the variable is a vector (small bold letter) or a matrix (capital bold letter). We gather all the $x$ 's into an $M x N$ matrix $\mathbf{X}$ and the $y$ 's into a $M x 1$ vector $\mathbf{y}$ and the weights $1 x N$ vector $\mathbf{w}$. The weight vector is also quite often called $\boldsymbol{\beta}$.

$$
\underbrace{\boldsymbol{y}}_{\mathrm{Mx} 1}=\underbrace{\boldsymbol{X}}_{\mathrm{M} \times \mathrm{N}} * \underbrace{\boldsymbol{w}}_{\mathrm{N} \times 1}
$$

Equation 4.29 describes the predicted $y$ values given the $X$ 'es and the weight vector, but to make the equation complete we must include the error of the prediction. This error is usually called $\sigma$ or $\epsilon$ and is assumed to be normally distributed with a zero mean. The complete equation then comes out as:

$$
\underbrace{\boldsymbol{y}}_{\mathrm{Mx} 1}=\underbrace{\boldsymbol{X}}_{\mathrm{MxN}} * \underbrace{\boldsymbol{w}}_{\mathrm{Nx} 1}+\underbrace{\boldsymbol{\sigma}}_{\mathrm{Mx} 1}
$$


This setup can be visualized as follows:

$$
\left[\begin{array}{c}
y_{1} \\
y_{2} \\
\vdots \\
y_{M}
\end{array}\right]=\left[\begin{array}{cccc}
1 & x_{1,1} & \cdots & x_{1, N} \\
1 & x_{2,1} & \cdots & x_{2, N} \\
\vdots & \vdots & \ddots & \vdots \\
1 & x_{M, 1} & \cdots & x_{M, N}
\end{array}\right] *\left[\begin{array}{llll}
w_{1} & w_{2} & \cdots & w_{N}
\end{array}\right]^{T}+\left[\begin{array}{c}
\sigma_{1} \\
\sigma_{2} \\
\vdots \\
\sigma_{M}
\end{array}\right]
$$

In 4.31 the $T$, means transpose, which is the action of turning a column vector into a row vector and vice versa. We can also transpose a matrix by rotating its values around the diagonal. As in the single variable case we want to minimize the squared error of this equation. We have gathered the errors in the $\sigma$ column, so analogous to the single variable case, the squared error is:

$$
\sigma^{T} \sigma=\left[\begin{array}{c}
\sigma_{1} \\
\sigma_{2} \\
\vdots \\
\sigma_{M}
\end{array}\right]^{T} *\left[\begin{array}{c}
\sigma_{1} \\
\sigma_{2} \\
\vdots \\
\sigma_{M}
\end{array}\right]=\sum_{i=0}^{M} \sigma_{i} * \sigma_{i}
$$

If we solve for $\sigma$ in Equation 4.30 we get

$$
\sigma=y-X * w
$$

and thus the SSE is

$$
\sigma^{T} \sigma=(y-X * w)^{T}(y-X * w)
$$

As in the single variable case we want to minimize the SSE. To get 4.32 in a form that is suitable for minimization we use rules 4.33 and 4.34 of matrix algebra:

$$
\begin{gathered}
(y-X * w)^{T}=y^{T}-(X * w)^{T} \\
(X * w)^{T}=X^{T} * w^{T}
\end{gathered}
$$

to get

$$
\sigma^{T} \sigma=\left(y^{T}-X^{T} * w^{T}\right)(y-X * w)
$$

Going from 4.35 to 4.37 we use the matrix algebra relation:

$$
X^{T} * w^{T} * y=y^{T} * X * w
$$

Other than catering for 4.36 we can think of going from 4.35 to 4.37 as the normal single variable cross multiplication of the terms in 4.35 .

$$
\sigma^{T} \sigma=\left(y^{T} y-y^{T} * X * w-X^{T} * w^{T} * y-y^{T} * X * w+X^{T} * w^{T} * X * w\right)
$$


Gather the terms in 4.37

$$
\sigma^{T} \sigma=\left(y^{T} y-2 * w^{T} * X^{T} * y+w^{T} * X^{T} * X * w\right)
$$

If we compare Equation 4.38 with the single variable case where we substitute Equation 4.6 for $\hat{y}$ in Equation 4.8 we get

$$
e^{2}=(y-(\text { slope } * x))^{2}
$$

We may recognize this as a square formula from algebra, it can be expanded to

$$
e^{2}=(y-(\text { slope } * x)) *(y-(\text { slope } * x))
$$

We can then perform the cross multiplications and gather the different terms

$$
e^{2}=y^{2}-2 * \text { slope } * x * y+(\text { slope } * x)^{2}
$$

We see by comparing Equations 4.41 and 4.38 that the single variable derivation is analogous to the multivariate case aside from the fact that in the linear algebra case we must cater for the dimensions of the interacting variables. So the $y_{i}^{2}$ term in the single variable case corresponds to $\boldsymbol{y}^{\boldsymbol{T}} \boldsymbol{y}$ in the linear algebra setup, the $\mathbf{2} *$ $\boldsymbol{w}^{\boldsymbol{T}} * \boldsymbol{X}^{\boldsymbol{T}} * \boldsymbol{y}$ corresponds to $2 *$ slope $* x_{i} * y$ and $\boldsymbol{w}^{\boldsymbol{T}} *$ $\boldsymbol{X}^{\boldsymbol{T}} * \boldsymbol{X} * \boldsymbol{w}$ to $\left(\text { slope } * x_{i}\right)^{2}$.

The Square and Completing the Square

The 2:nd degree polynomial $(x+a)^{2}$ can be expanded to the form $x^{2}+$ $2 a x+a^{2}$. Going from this latter form to the former is called completing the square. In Equations 4.394.41 we use versions of completing the square. In Section 4.4 we will be using the analog matrix version of completing the square.

Analogous to the single vari-

able case we take the derivative of the squared error terms with respect to the weight vector. To do this we use the following identities from linear algebra

$$
\begin{gathered}
\frac{\partial y^{T} y}{\partial w}=0 \\
\frac{\partial w^{T} * X^{T} * y}{\partial w}=X^{T} * y \\
\frac{\partial w^{T} * X^{T} * X * w}{\partial w}=2 * X^{T} * X * w
\end{gathered}
$$


Again, analogous to the single variable case we set the derivative to 0

$$
0=0-2 * X^{T} * y+2 * X^{T} * X * w
$$

Rearranging this we get

$$
X^{T} * X * w=X^{T} * y
$$

And again we solve for $\boldsymbol{w}$

$$
\begin{gathered}
\underbrace{\left(X^{T} * X\right)^{-1} * X^{T} * X}_{\text {analogous to } \frac{x^{2}}{x^{2}}=1} * w=\left(X^{T} * X\right)^{-1} * X^{T} * y \\
w=\left(X^{T} * X\right)^{-1} * X^{T} * y
\end{gathered}
$$

Equation (4.48) is usually called the normal equation of the OLS, where $\left(\boldsymbol{X}^{\boldsymbol{T}}\right.$ * $\boldsymbol{X})^{-1} * \boldsymbol{X}^{\boldsymbol{T}}$ is called the MoorePenrose pseudoinverse of X. The Penrose part of MoorePenrose is the famous British mathematician Sir Roger Penrose. In the matrix algebra case we left multiply and raise to the negative power (i.e. inverting the matrix, although in general we have to be careful because not all matrices can be inverted) rather than dividing. This final answer can be seen to be analogous to the single variable case in Equation 4.19 which is repeated here for reader convenience.

$$
\text { slope }=\left(\sum_{i=1}^{N} x_{i} * x_{i}\right)^{-1} * \sum_{i=1}^{N}\left(x_{i} * y_{i}\right)
$$

If we apply Equation 4.48 to the data from Table 4.1, including adding the column of ones for the intercept to the $\boldsymbol{X}$ matrix we get the vector $[8.42$, $0.072]$ where the first element (8.42) is the intercept and the second element (0.072) is the slope of the regression line. This result corresponds to the green-dashed regression line in Figure 4.8, which has a slightly smaller slope (0.071 vs. 0.095$)$ compared to the single variable purple line and an intercept which crosses the y-axis at 8.42 vs. the purple line that we forced to cross at zero. The data was in fact generated from an equation with intercept 10 and slope 0.07 with added noise from a Gaussian distribution with mean zero and standard deviation of 5 . So by adding the intercept we get a better fit to our data. The SSE has improved from 426 to 133 (a smaller error is better).

There is a lot more to regression analysis that we do not have the possibility to go into detail on here but we will mention one aspect that is of major interest. Once the regression analysis is done, we are interested in the so called $R^{2}$ and Adjusted $R^{2}$ measures. The $R^{2}$ tells us, in percent, how much of the variance in $y$ that is explained by our regression, i.e. the predicted $\hat{y}$ we get when we use our regression equation. The $R^{2}$ is used as a measure of how good a job our regression performs. The more variance that is explained 
by $R^{2}$, the closer to one the $R^{2}$ is, the better is our regression. The $R^{2}$ value of the full regression including the intercept is 0.83 . This can be compared with the $R^{2}$ of the single variable case which is 0.69 . The Adjusted $R^{2}$ is an adaptation of the $R^{2}$ measure which takes into account the number of covariates and the number of samples used in the regression equation. This gives a more fair measure of the true power of the regression, since in general, adding more covariates will improve the $R^{2}$ but not always the predictive power of the regression due to over fitting.

\section{From Linear Regression to Binary Classification Using Logistic Regression}

We showed in the previous section how we can learn the parameters of a linear regression so we are able to predict for instance future CPU loads given how many users are connected to a system. In this section we give an overview of how linear regression can be built upon to obtain other models. What if our goal is instead to do a classification or more specifically a binary classification. That is, instead of having a table with a real number responses we have binary responses such as in Table 4.2.

\begin{tabular}{rrr}
\hline & $\mathrm{y}$ & $\mathrm{x}$ \\
\hline 1 & 0 & 290.0 \\
2 & 1 & 490.0 \\
3 & 0 & 250.0 \\
4 & 1 & 460.0 \\
5 & 1 & 360.0 \\
6 & 0 & 230.0 \\
7 & 0 & 67.0 \\
8 & 0 & 130.0 \\
9 & 0 & 11.0 \\
10 & 1 & 440.0 \\
\hline
\end{tabular}

Table 4.2: 10 observations of the binary-valued variable $y$ given inputs $x$

In this case we might instead want to predict if the system gets overloaded (true $=1$ or false $=0$ ) depending on the number of users. To model this we can extend the linear regression to a model that is called logistic regression. In logistic regression we model the probability that the system overloads rather than the CPU load itself. This is done by running the linear regression through a transformation function called the sigmoid function. The sigmoid compresses the result from the linear regression range of $-\infty$ to $+\infty$ to the $0-1$ range. The definition of the sigmoid is as follows

$$
p(Y=1 \mid x)=\frac{1}{1+e^{-\left(w_{0}+w_{1} \star x\right)}}
$$


Equation 4.49 should be read as follows: "The probability that Y equals 1 given $\mathrm{x}$ is...". We recognize our old friend the linear equation in the exponent of the denominator. The learning task is then, as in the linear regression case, to find the weights $w_{0}$ and $w_{1}$ so that we get the best fit to our data. Unlike the linear regression this problem cannot be solved in closed form, i.e. we cannot find a formula for the minimum for the function as in the linear regression case. We have to solve this learning problem using numerical methods such as the Newton-Raphson method, the details of which we will not go into here. Analogous to the linear regression we can expand the logistic regression to take more input variables into consideration.

\section{From Binary Classification to Multiclass Classification}

We can further extend the logistic regression to a multiclass problem, where, instead of having just two classes (true and false) we can have $C$ classes. This is done by expanding the number of parameters in the model. In the binary logistic regression we managed with one set of parameters where the parameters were used to calculate the probability of a success. The probability of a fail is then just $1-p$ (success) so we get both cases from one set of parameters. In multiple logistic regression we have one set of parameters per class and the probability that an observation belongs to class $c$ is formulated as

$$
p(Y=c \mid x)=\frac{e^{-\left(w_{0}^{(c)}+w_{1}^{(c)} * x\right)}}{\sum_{c=1}^{C} e^{-\left(w_{0}^{(c)}+w_{1}^{(c)} * x\right)}}
$$

Once we have learned the parameters $w$, we can perform a classification under this scheme by calculating the probability for each of the classes using 4.50 and choose the class with the highest probability.

\section{Multiclass Classification using Naive Bayes}

In the above sections we have explored learning algorithms based on linear regression. In this section we look at a different approach to classification called Naive Bayes. The appeal of Naive Bayes lies in its intuitive approach in combination with its quite robust performance.

To make the discussion concrete we will use an imaginary bug database as an example. We will encode the database with four variables. These are Component, Site, Customer and, Priority. Each variable has a set of possible states, for instance the Site variable have the states Site1, Site2, etc. The imagined system have four states for the Component variable, the UI, the Database, the Controller and the Business logic. Table 4.3 shows an example of how such a bug database may look like.

The intuition behind Naive Bayes can be explained by looking at Table 4.4 which contains a summary of Table 4.3. In Table 4.4 we look at the Site 
variable and summarize how many bugs on each Component comes from each site. For instance, 324 bug reports was reported from Site 5 and on the UI component.

We can look at the data from Table 4.4 in two ways. If we only look at the row for Site 5, we would say that if the bug report is from Site 5 there is a $324 /(324+29+4+0)=0.907$ i.e. ca $91 \%$ chance that the bug is in the UI component. Correspondingly, there is a $29 /(324+29+4+0)=0.081$ i.e. ca $8 \%$ chance that the bug is in the Database component. We have now calculated

$$
p(\text { Component }=U I \mid \text { Site } 5) \approx 90 \%
$$

and

$$
p(\text { Component }=\text { Database } \mid \text { Site } 5) \approx 8 \%
$$

We can do a similar analysis for the other variables. Using this reasoning we get an estimate of $p$ (Component|variable). It is now tempting to conclude, "We are interested in the probability of the bug belonging to component $C$ given the input variables (Site, Customer, Priority etc), so we'll just multiply together the probabilities for the component given each of the different variables". We could now argue that we have calculated the probability of a component given all of the variables. The problem with this approach is that we fail to take into account the general prevalence of the component in the dataset as a whole. This measure is sometimes called the base rate, and the mistake of ignoring it is called base rate neglect. The problem with base rate neglect is that we tend to overestimate the probability of low occurring classes (Components in our case) in the data. For instance, the base rate of the Controller component is only $43 / 2452=0.0175 \approx 18 \%$.

\begin{tabular}{|l|l|l|l|l|l|l|}
\hline \multicolumn{7}{|c|}{ Bug Database } \\
\hline Bug Id & Site & Customer & Priority & Heading & Description & Component \\
\hline \hline BugId-01 & Site 1 & Customer 1 & Priority 3 & No access & $\begin{array}{l}\text { Cannot access } \\
\text { the database... }\end{array}$ & Database \\
\hline BugId-02 & Site 2 & Customer 4 & Priority 1 & No sync & $\begin{array}{l}\text { Sync is lost be- } \\
\text { tween... }\end{array}$ & Controller \\
\hline BugId-4709 & Site 1 & Customer 12 & Priority 1 & $\begin{array}{l}\text { Wrong } \\
\text { contract }\end{array}$ & $\begin{array}{l}\text { Wrong con- } \\
\text { tract is re- } \\
\text { turned... }\end{array}$ & $\begin{array}{l}\text { Business } \\
\text { logic }\end{array}$ \\
\hline BugId-4710 & Site 4 & Customer 3 & Priority 3 & $\begin{array}{l}\text { Missing } \\
\text { menu }\end{array}$ & $\begin{array}{l}\text { Menu not visi- } \\
\text { ble... }\end{array}$ & UI \\
\hline BugId-4711 & Site 5 & Customer 1 & Priority 2 & Backup & Replication ... & Database \\
\hline
\end{tabular}

Table 4.3: A simplified imaginary database of bug reports. Ellipsis at the end of descriptions symbolizes that a longer text follows. 
Now we will take another approach to remedy this problem. Another way to look at the data in Table 4.4 is to look at it column wise. By column, there is a

$$
324 /(5+89+13+167+324)=0.541 \approx 54 \%
$$

chance that it is Site 5 that has reported a bug given that we know it is the UI component, that is, we calculate $p($ Site $5 \mid$ Component $=U I)$ instead of $p($ Component $=U I \mid$ Site 5$)$. We can also calculate the general prevalence of the UI in the dataset $p($ Component $=U I)$, this is $598 /(598+211+43+1600)=$ 0.243 , roughly $24 \%$. Now we can use Bayes rule which in its general form says:

$$
p(A \mid B)=\frac{p(B \mid A) p(A)}{p(B)}
$$

and specifically its proportional form which says:

$$
p(A \mid B) \propto p(B \mid A) p(A)
$$

This translates in our example to

$p($ Component $=U I \mid$ Site 5$) \propto p($ Site $5 \mid$ Component $=U I \mid) p($ Component $=U I)$

where we take the base rate of the UI component into consideration with the $p($ Component $=U I)$ part of the rule. We can now calculate the proportional probability of all components and select the component with the highest probability to be our classification! But wait, we have more variables than just the Site variable, how about the Customer, Priority etc? Here is where the

\begin{tabular}{|c|c|c|c|c|c|}
\hline & \multicolumn{4}{|c|}{ Component } & \\
\hline Site & $\overline{U I}$ & Database & Controller & $\begin{array}{l}\text { Business } \\
\text { logic }\end{array}$ & Total \\
\hline Site 1 & 5 & 7 & 0 & 128 & 140 \\
\hline Site 2 & 89 & 81 & 26 & 1257 & 1453 \\
\hline Site 3 & 13 & 8 & 0 & 0 & 21 \\
\hline Site 4 & 167 & 86 & 13 & 215 & 481 \\
\hline Site 5 & 324 & 29 & 4 & 0 & 357 \\
\hline Total & 598 & 211 & 43 & 1600 & 2452 \\
\hline
\end{tabular}
Naive part of Naive Bayes comes in. The naive assumption is that we can just multiply the proportional probabilities of the different variables together

Table 4.4: Summary of bug reports from Table 4.3. The numbers in the cells reports the total number of bug reports from each site on each component. In this example Site 2 has reported 26 bugs on the Controller component. 
and plug in to the formula. This is called naive because we are doing an independence assumption between the variables, even though we know they are not independent. As an example, consider that if we know which customer reported a bug that will give us some quite strong hints on which site the bug was reported on, i.e. the Customer and Site variables are not independent. That is, the general quantity that we are interested in is, the probability of a component given all of the variables, we can express this as:

$p($ Component $\mid$ variables $) \propto p($ variables $\mid$ Component $) p($ Component $)$

where variables means all of the variables in the bug report (Site, Customer, Priority, Heading and Description). This would, with the naive assumption, translate to:

$$
p(C \mid S, C u, P, H, D) \propto p(S \mid C) p(C u \mid C) p(P \mid C) p(H \mid C) p(D \mid C) p(C)
$$

where we have abbreviated the variables to the first letter in the variable name aside for the following two exceptions, $C=$ Component and $C u=C u s t o m e r$. All of the expressions in 4.53 can be calculated from our dataset the same way as in 4.51 .

We can generalize the Naive Bayes formulation as follows

$p(Y=c \mid \boldsymbol{X}) \propto p\left(X_{1}=s_{x_{1}} \mid Y=c\right) p\left(X_{2}=s_{x_{2}} \mid Y=c\right) \ldots p\left(X_{P}=s_{x_{p}} \mid Y=c\right) p(Y=c)$

Where $\boldsymbol{X}$ is a $P$ dimensional vector and $p\left(X_{2}=s_{x_{2}} \mid Y=c\right)$ means the probability that the second variable in $\boldsymbol{X}$ takes the state $s_{x_{2}}$ given that the class of $Y$ is $c$. In the simplest Naive Bayes this is typically calculated using the so called maximum likelihood estimate (MLE). The maximum likelihood estimate of $p(Y=c)$ is simply the number of observations that have class $c$, we denote this sum $c_{c}$ for class $c$, divided by the total number of observations in the dataset, which is $N$. The MLE for $p\left(X_{p}=s \mid Y=c\right)$ is the number of times variable $X_{p}$ took state $s$, when the class was $c$, we denote this value $C^{c, x_{p}}$, divided by $c_{c}$. So the generic version of Equation 4.54 becomes

$$
p(Y=c \mid \boldsymbol{X}, \boldsymbol{D a t a}) \propto \frac{c_{c}}{N} \prod_{p} \frac{C^{c, x_{p}}}{c_{c}}
$$

Here the $c$ counts come from the historic data and $s=x_{p}$ comes from the new $\boldsymbol{X}$ that we want to predict.

There are some problems with the Naive Bayes approach we have described up to this point that needs to be overcome. One problem occurs when we have sparse data. For instance, if we look in Table 4.4 at the Controller component and Site 3 we see that we have zero counts. This means that when we calculate 
$p($ Site $3 \mid$ Component $=$ Controller $)$ we get $0 /(0+26+0+13+4)=0$, i.e. we get a zero \% chance of Site 3 given that the component is the Controller. This means that the whole nominator in 4.53 will be zero and the final probability also zero. This is a manifestation of the problem with sparsity, i.e. we might not have observations of all possible combinations of data. This problem is magnified with higher number of dimensions in the problem context. This is probably not what we want, since even though we have not yet seen a bug report from Site 3, we might still believe it is a possibility in the future. This problem is usually dealt with by employing some kind of smoothing scheme. The most common smoothing scheme is the Laplace smoothing scheme. With Laplace (also called "add-one") smoothing, we simply add one to each of the counts in the whole database. We will see later that Naive Bayes with Laplace smoothing is a special case of Bayesian Naive Bayes, a more principled way of doing Naive Bayes where we get the smoothing for "free" as part of the general Bayesian approach.

This smoothing scheme adds one for each class to $c_{c}$ and similarly for each $C^{c, x_{p}}$ (irrespective if we have observed a value or not) and thus we need to compensate for that in the corresponding denominator. Which gives us

$$
Y \leftarrow c=\underset{c}{\arg \max } p(Y=c \mid \boldsymbol{X}, \boldsymbol{D a t a}) \propto \frac{1+c_{c}}{k+N} \prod_{p} \frac{1+C^{c, x_{p}}}{C_{p, s}+c_{c}}
$$

Where $k$ is the number of classes and $C_{p, s}$ is the number of states for variable $X_{p}$.

Another problem with MLE estimates is overfitting, this is also dealt with in the Bayesian approach by using priors.

We can now make up a new $\boldsymbol{X}$ and use our two equations to predict the different class probabilities. Table 4.5 lists the class probabilities for the different components calculated using Equation 4.55 and Equation 4.56 for a new made up a new bug report with, Customer $=$ Customer 5, Country = Country3, Priority $=$ Priority 2 , and Site $=$ Site 3 .

So far we have discussed the Naive Bayes approach to classification in the case of categorical data such as Customer, Site etc, but the approach can be adapted to contain a mix of other input types such as binary, real valued data, and count data. We have also not discussed how the textual inputs are handled. In the most basic case, text is modeled using a technique called TF-IDF.

In TF-IDF text modeling, each word in a text is given a score. The score is based on two components; how many times a word occurs in the document and how many times the word occurs in the whole corpus. The intuition behind TF-IDF is that if a word occurs many times in a document it is important, but the importance is reduced if it also occurs many times in the whole corpus. If the word occurs many times in the whole corpus it is likely not an informative 


\begin{tabular}{|l|l|l|l|l|}
\hline & \multicolumn{3}{|c|}{ Component } \\
\hline Fit Type & $\begin{array}{l}\text { Controller } \\
\%\end{array}$ & $\begin{array}{l}\text { Database } \\
\%\end{array}$ & $\begin{array}{l}\text { UI } \\
\%\end{array}$ & $\begin{array}{l}\text { Business } \\
\text { logic \% }\end{array}$ \\
\hline $\begin{array}{l}\text { Maximum } \\
\text { Likelihood } \\
\text { Estimate } \\
\text { (Eq. 4.55) }\end{array}$ & 0 & 87 & 1 & 13 \\
\hline $\begin{array}{l}\text { Smoothing } \\
\text { (Eq. 4.56) }\end{array}$ & 3 & 88 & 1 & 9 \\
\hline
\end{tabular}

Table 4.5: Class probabilities in percent (not summing to 100 due to rounding) using Naive Bayes fitted using MLE and Laplace Smoothing (Equation number in parenthesis).

word, typically these are words like "a", "and", "the" etc. There are many variants of TF-IDF but one of the simplest is the following:

$$
t f i d f=f_{w, d} * \log \left(\frac{N}{n_{w}}\right)
$$

where $f_{w, d}$ is the frequency of word $w$ in document $d, N$ is the number of documents in the corpus and $n_{w}$ is the number of documents in the corpus that the word $w$ occurs.

Using this approach we can score the words in the documents and use the TF-IDF score as a numerical input to our Naive Bayes classifier. This means that the textual inputs to the algorithm will be expanded from one text variable to one variable per unique word in the corpus. This will create so called wide data, i.e. we have many columns in our dataset. A substantial part of this thesis is dedicated to better modeling of unstructured text. In Paper III and VI we develop a fast exact method for inference in the LDA model. In Paper IV we extend this method to a classification technique that can handle both categorical and numerical data in combination with unstructured text. Paper $\mathrm{V}$ then studies how we can use this more sophisticated text modeling technique to get concrete benefits in an applied setting.

\subsection{Bayesian Learning}

Bayesian learning $^{2}$ is a beautiful and general mathematical theory which in principle allows a machine to learn from experience. Specifically, this is done by specifying some prior belief on the parameters of interest (for instance the weights $w$ in our linear regression example in Section 4.3), observing some data, and finally, formally updating the prior belief to arrive at a posterior distribution on the parameters. The beauty of the Bayesian approach is that

\footnotetext{
${ }^{2}$ Also called Bayesian Statistics or Bayesian Inference
} 
we don't just get a point estimate of the parameters of interest, we get a full probability distribution for all possible values of the parameters.

This is done via the so called Bayes Rule, which we mentioned briefly before, which we will now study in more detail. Bayes rule is derived from the basic axioms of probability theory. The chain rule of probability theory says that:

$$
p(A, B)=p(A \mid B) p(B)
$$

Where $p(A, B)$ means that both events $A$ and $B$ occurs and $p(A \mid B)$ mean the probability that $A$ occurs given that we know that $B$ has occurred. Since $p(A, B)$ is symmetric, i.e. $p(A, B)=p(B, A)$ we have that:

$$
p(B, A)=p(B \mid A) p(A)=p(A, B)
$$

If we combine 4.58 and 4.59 we get that:

$$
p(A \mid B) p(B)=p(B \mid A) p(A)
$$

Which can be re-arranged to yield Bayes Rule:

$$
p(A \mid B)=\frac{p(B \mid A) p(A)}{p(B)}
$$

A few points need to be made regarding components of 4.61. In the context of Bayes Rule the expression $p(A)$ is called the prior or the prior on $A \cdot p(B)$ (which we assume is non-zero) is called the evidence and $p(B \mid A)$ is called the likelihood or the likelihood function. Finally $p(A \mid B)$ is called the posterior or the posterior on A given B. Not all of the components of Bayes Rule are always needed. For instance, often we only need something that is proportional to the posterior, so we can simplify Bayes rule to:

$$
p(A \mid B) \propto p(B \mid A) p(A)
$$

Equation 4.62 should be read, the posterior on $A$ given $B$ is proportional to the likelihood of $B$ given $A$, times the prior on $A$. This is often useful since $p(B)$ (the denominator that we are ignoring) can be very hard to calculate. For full Bayesian inference we always need all of the components in 4.62, and defining the likelihood function, selecting the prior and deriving an expression for the posterior is at the heart of Bayesian Learning. In the context of machine learning Equation 4.62 is often stated in another language. In machine learning we very often want to learn something about the parameters of a model given some data. In the linear regression model, the parameters are the weight vector $w$. Equation 4.62 is then read as follows, the probability of the parameters is proportional to the data likelihood and the prior. I.e we want to learn the parameters of the model given some data. 
The chain rule can be generalized to:

$$
p\left(A_{1}, A_{2}, \ldots A_{p}\right)=p\left(A_{1}\right)\left(A_{2} \mid A_{1}\right) p\left(A_{3} \mid A_{2}, A_{1}\right) \ldots P\left(A_{p} \mid A_{p-1}, . ., A_{1}\right)
$$

The generalization of the chain rule allows us to do manipulations of Bayes rule when we have more variables involved, such as:

$$
p(A, B \mid C, D)=\frac{p(C, D \mid A, B) p(A, B)}{p(C, D)}
$$

Which can be transformed to:

$$
p(A, B \mid C, D)=\frac{p(D \mid A, B, C) p(C \mid A, B) p(A, B)}{p(C, D)}
$$

and

$$
p(A, B \mid C, D)=\frac{p(D \mid A, B, C) p(C \mid A, B) p(B \mid A) P(A)}{p(C, D)}
$$

Depending on the concrete meanings of the events $A, B, C, D$ these transformations can be very useful in reducing the complexity and usefulness of the function. We will see examples of these types of manipulations later in this chapter.

While Bayes Rule is straight forward to derive and looks deceptively simple, there is a sophisticated mathematical theory behind the scenes, with many subtleties and nuances. When we start modeling more complex problems, the involved distributions make the mathematical theory quite complex. The full theory involves exotic probability distributions and the mathematical rules for how to manipulate them. Further, to actually calculate something useful using Bayesian statistics we need software libraries that are numerically very stable and robust, since extreme values can be quite common in Bayesian inference. Specifying a Bayesian model can be comparatively easy, but deriving an actual sampler for the learning problem can be quite a complex task and require substantial mathematic skills. In particular, knowledge of integration theory and Markov chain Monte Carlo sampling theory is a necessary skill for many Bayesian learning problems.

There are frameworks (for example BUGS, WinBUGS, OpenBUGS and Stan) which allows the user to specify the Bayesian model, and the framework will then create a sampler for that model. These frameworks use general classes of inference to implement the sampler. Unfortunaltely, these general classes are still today not efficient enough to solve many problems involving complex models and large data sets. For these problems specialized samplers needs to be derived by using domain knowledge about the problem. The derivations uses probability theory to manipulate the resulting probabilistic mathematical functions and computer science to parallelize the resulting code. 
In combination, this means that implementing a fast parallel sampler for complex models and large data sets, requires expert knowledge in the mathematics of sampling and probability theory, and computer science. Even with tools like BUGS and Stan specialized skills and tricks, typically not available in industry, are needed to make the model run fast enough.

Our third, fourth and sixth paper concerns these problems. In the third paper we develop an exact parallel sampler for the LDA model. In the sixth paper, we further improve the effectiveness of our sampler in paper three. In the fourth paper we expand on the LDA model and develop a supervised classification model for unstructured text, which we call DOLDA, which can also be augmented with additional structured covariates.

\section{Bayesian Building Blocks - A Hierarchy of Discrete Probability Distributions and their Conjugate Priors}

One of the great advantages with the Bayesian paradigm is that we can use probability theory and some basic building blocks in the form of probability distributions, and put them together, much like Lego. Probability theory lets us do this in a mathematically consistent manner to get a probabilistic model of our problem. In the following sections, we discuss some of the very important building blocks for Bayesian inference in discrete models. These building blocks are probability distributions used to model aspects of the world we want to learn about. Probability theory supplies us with a large body of distributions suitable for modeling various aspects of the real world, from simple success / failure, waiting times between events, to probability distributions over probability distributions.

Before diving into the theory we must introduce the concept of conjugacy. Conjugacy is a very important concept in Bayesian learning. A conjugate prior distribution is a distribution which exact mathematical formulation, when combined with the likelihood expression, will give us a posterior distribution which is of the same mathematical form as the prior. This has the advantage of giving comparatively simple mathematical rules for updating our prior to get the posterior. In the sections below, we show examples of some common distributions and their conjugate priors.

\section{Beta-Bernoulli}

The Bernoulli distribution is one of the simplest distributions around but still very important. Its importance lies in it being a basic building block for more advanced models. In a sense we build up from the Bernoulli to more advanced discrete distributions. The Bernoulli models a one-off experiment with outcomes success or failure, the classical example being a simple coin flip. How we define "success" and "failure" is up to us. It could be a patient surviving a surgery, it could be guessing correct on a taste test, or a coin 
coming up heads. The Bernoulli is also used for variable selection in more complex Bayesian models. In the variable selection case, the Bernoulli random variable indicates if a variable in the model should be kept or not. We use this in Paper III [35] to show that our correct and parallel LDA implementation allows for variable selection were other models do not.

The Bernoulli has one parameter often called $\theta$ which expresses the probability of success. We say that $X$ is Bernoulli distributed with parameter $\theta$.

$$
X \sim \operatorname{Bern}(\theta)
$$

Where the tilde symbol $\sim$ should be read as distributed as or distributed according to. The probability mass function for the Bernoulli is expressed in Equation 4.68 .

$$
p(X=x \mid \theta)=\theta^{x}(1-\theta)^{(1-x)}
$$

True to our Bayesian approach, we need a prior on $\theta$. Since the $\theta$ parameter of the Bernoulli expresses a probability and hence is restricted to the 0-1 range, the best prior distribution for that parameter is one that is naturally limited to the same range. Ideally we also want one that is conjugate. The Beta distribution fulfills these exact requirements. We can express $\theta$ as a Beta random variable as follows

$$
\theta \sim \operatorname{Bet} a(a, b)
$$

The probability density function ( $\theta$ is a real valued number limited to the $0-1$ range, thus we have a pdf for $\theta$ rather than a pmf) for the Beta is expressed in Equation 4.70.

$$
p(\theta \mid a, b)=\frac{\theta^{(a-1)}(1-\theta)^{(b-1)}}{B(a, b)}
$$

Here $B(a, b)$ is the so called beta function, which is not a probability distribution but an ordinary mathematical function. Now we have a likelihood function (the Bernoulli) and a prior (the Beta), we now combine them to the Beta-Bernoulli model. With the Beta-Bernoulli we want to calculate the posterior probability of the parameter $\theta$ given some data and our prior beliefs. Our data is the one experiment (one coin flip or more general, one success or failure). We recall from Bayes rule that to calculate the posterior, we multiply the likelihood with the prior and divide with the evidence. In this case the Bernoulli is the likelihood and the Beta is the prior. So true to the Bayesian dictum of use Bayes rule we get

$$
p(\theta \mid X)=\frac{p(\theta) p(X \mid \theta)}{p(X)}
$$


Now we resort to our "trick" of ignoring the normalizing denominator which is constant with respect to $\theta$ (i.e., $\theta$ is not part of the expression for the denominator and thus, it does not depend on $\theta$ ). With this we get

$$
p(\theta \mid X) \propto p(\theta) p(X \mid \theta)
$$

Plugging in the distributions for the prior and the likelihood we get

$$
p(\theta \mid X) \propto \operatorname{Beta}(a, b) \operatorname{Bern}(\theta)
$$

This is the posterior proportional to prior times likelihood form of Bayes rule (where we ignore normalization constants), that we saw in Equation 4.62. Plugging in the function for the Beta and the Bernoulli into 4.73, we get

$$
p(\theta \mid X=x) \propto \theta^{(a-1)}(1-\theta)^{(b-1)} \theta^{x}(1-\theta)^{(1-x)}
$$

From now on we simplify write $\mid X$ instead of the full $\mid X=x$, but note the difference between small and capital $X$ 's. Gather the factors, i.e. since multiplication is symmetric we can move the factors containing $\theta$ and $1-\theta$ next to each other

$$
p(\theta \mid X) \propto \theta^{(a-1)} \theta^{x}(1-\theta)^{(b-1)}(1-\theta)^{(1-x)}
$$

Sum the exponents of $\theta$ and $1-\theta$

$$
p(\theta \mid X) \propto \theta^{(a-1+x)}(1-\theta)^{(b-1+1-x)}
$$

At this stage we can ask; Why not simplify the exponents in the second factor? It turns out that keeping them and re-arranging the terms will lead to a revelation. So re-arranging the terms in the exponents we get

$$
p(\theta \mid X) \propto \theta^{(a+x-1)}(1-\theta)^{(b+1-x-1)}
$$

If we study the form of Equation 4.75 and compare it with the equation for the Beta distribution (Equation 4.70) we see that it is actually again a Beta distribution, with $a$ replaced with $a+x$ and $b$ replaced with $b+1-x$, but without the normalization constant. This in turn gives us the normalizing constant that we ignored in Equation 4.73. Since we already know that the normalizing constant for a $\operatorname{Bet} a(a, b)$ is $B(a, b)$, then the normalizing constant for a $\operatorname{Beta}(a+x, b+1-x)$ must be $B(a+1, b+1-x)$. Which gives our exact posterior after having seen one event

$$
p(\theta \mid X)=\frac{\theta^{((a+x)-1)}(1-\theta)^{((b+1-x)-1)}}{B(a+x, b+1-x)}
$$

This is an elegant result, since we get our posterior by just plugging in the value of the outcome in 4.76 ! Since it is again a beta distribution we can use all the known properties of the beta distribution for calculating properties 
such as the posterior mean, variance and all other properties we might be interested in.

One might now ask; Why not use a uniform distribution as the prior for $\theta$ since it is simpler and perhaps more intuitive? There are many answers to that question. First, the uniform is not conjugate to the Bernoulli, so we would not get one of the standard distributions back (by using the Beta, we got a standard Beta as the posterior). This has the downside that we cannot just lookup the properties, such as its mean, median, mode, variance, etc. of that distribution. Second, there typically exist no ready library functions for drawing samples from these non-standard distributions, something we often want to do in Bayesian inference when we use MCMC. Third, the uniform is a special case of the Beta distribution, so we can easily model a uniform distribution by setting $a=b=1$ in the Beta distribution. In addition, the Beta is flexible in the sense that we can model sophisticated prior knowledge with the Beta such as, our prior assumptions of the mean and the variance of $\theta$. For instance, if we have strong prior opinions of $\theta$ we can select $a$ and $b$, so we get an mean of our choice and high values of $a$ and $b$ which will lead to smaller variance around the mean. We can use a uniform distribution if we don't care about an analytical solution, or we are using a framework such as BUGS or Stan.

\section{From the Bernoulli to the Binomial distribution}

The Bernoulli only models one event. The advantage with the Bernoulli is that it is easy to understand and explain and at the same time easily extended to the case where we have several events, for instance many coin flips, or many patient cases. To extend the Bernoulli to many cases, we make two underlying assumptions, the cases are considered independent trials, i.e. one case does not affect the outcome of another, and the probability of success is the same in each of the trials. This can be stated as follows

$$
X_{1}, X_{2}, \ldots X_{N} \sim \operatorname{Bern}(\theta)
$$

If each of the $X_{n}$ is an independent Bernoulli trial, the rules of probability says that we can multiply their individual probabilities to get the joint probability of one particular sequence of events.

$$
p\left(X_{1}, . . X_{N} \mid \theta\right)=\prod_{1}^{N} \theta^{x_{n}}(1-\theta)^{\left(1-x_{n}\right)}
$$

were we have plugged in the probability function of the single Bernoulli trial. If we sum the exponents in Equation 4.78 we get

$$
p\left(X_{1}, . . X_{N} \mid \theta\right)=\theta^{\sum x_{n}}(1-\theta)^{N-\sum x_{n}}
$$


Let us now introduce a variable $k$ which is the number of successes in the sequence, $\sum x_{n}=k$ (remember that the $x_{n}$ 's only takes the values 0 or 1 ). We can now write Equation 4.79 as

$$
p\left(X_{1}, . . X_{N} \mid \theta\right)=\theta^{k}(1-\theta)^{N-k}
$$

Where $k$ is the number of successes and $N-k$ the number of failures. Now we are getting close to the normal extension of the Bernoulli model, the so called Binomial model. But the Binomial model answers a slightly different question than the Bernoulli. It answers the question; What is the probability of getting $S=k$ successes out of $N$ independent Bernoulli trials were each success has probability $\theta$ ? That is, we don't really care about the specific order of the trials, we are only interested in how many successes we get in all of our trials, irrespective of order. We say that $S$ is Binomially distributed

$$
S \sim \operatorname{Binom}(N, \theta)
$$

The Binomial distribution builds on the Bernoulli according to the following reasoning. Equation 4.80 tells us the probability of one particular sequence, or equivalently the probability of one particular way of getting $\operatorname{sum}\left(X_{1}, \ldots X_{n}\right)=$ $k$ successes. But since we don't care about the order (i.e. the sequence $1,0,1,1$ is the same as 1,1,1,0 since both has three 1's and one zero) in which we get the $k$ successes, and there are $\left(\begin{array}{l}N \\ k\end{array}\right)$ (read as "from N choose k") such sequences, the expression for the Binomial distribution becomes

$$
p(S=k \mid \theta, N)=\left(\begin{array}{l}
N \\
k
\end{array}\right) \theta^{k}(1-\theta)^{N-k}
$$

Where the $\left(\begin{array}{l}N \\ k\end{array}\right)$ tells us how many combinations we can make by choosing $k$ items out of $N$. If we compare the expression for the Binomial in Equation 4.82 with the corresponding expression for the Bernoulli in Equation 4.68 and ignore the $\left(\begin{array}{l}N \\ k\end{array}\right)$ we see that the single $x$ in the exponent of the first factor is replaced with $k$, the number of successes. In the exponent of the second factor the one single case is replaced by the total number of cases $N$ and $k$. Otherwise the functions are identical.

\section{Beta-Binomial}

We know that the Beta distribution is conjugate to the Bernoulli, and since the form (i.e. excluding the normalization constant, and the specific exponents) of the Bernoulli and the Binomial are the same, we know that the Beta will also be conjugate to the Binomial distribution. When we combine the Binomial with the same Beta prior as in the Bernoulli case we get the Beta-Binomial model.

$$
p\left(\theta \mid X_{1}, . . X_{N}\right) \propto \operatorname{Beta}(a, b) \operatorname{Bin}(\theta)
$$


Plugging in the function for the Beta and Equation 4.82 for the Binomial into 4.83 (again ignoring normalization constants, thus making the expression proportional), we get

$$
p\left(\theta \mid X_{1}, . . X_{N}\right) \propto \theta^{(a-1)}(1-\theta)^{(b-1)} \theta^{k}(1-\theta)^{N-k}
$$

Where $k$ is the number of successes in the sequence, $\sum x_{n}=k$ (remember that the $X$ 'es take only the values 0 or 1 , where 1 represents success). The derivation of this model proceeds identically (starting from Equation 4.74) as in Bernoulli model with the difference that $x$ is replaced by $k$ in the first factors exponent, and the $1-x$ is replaced by $N-k$ in the second factors exponent. Doing the derivation leads us to

$$
p\left(\theta \mid X_{1}, \ldots X_{N}\right)=\frac{\theta^{((a+k)-1)}(1-\theta)^{((b+N-k)-1)}}{B(a+k, b+N-k)}
$$

Which is the posterior probability of $\theta$ after having seen $\mathrm{N}$ independent Bernoulli trials. Again we have a simple update rule, we get the posterior by plugging in the number of observed successes and the total number of trials in the data.

With the above approach we have turned the calculation of the posterior of $\theta$ into a "batch" calculation, i.e., we have the whole set of observations and sum the number of successes. But we could just as well have arrived at the same conclusion by repeatedly applying Bayes rule, for one observation at a time, starting with the Beta prior, and using the posterior (Beta) of one event as the prior for the next.

The parameters $a$ and $b$ of the Beta prior is often interpreted as prior counts or pseudo counts, i.e. we interpret $a$ as the number of successes and $b$ as the number of prior failures we have seen before the experiment. We can see that the uncertainty (i.e. the variance) in the posterior of $\theta$ decreases the more number of observations we see. This is explained by looking at Equation 4.85, which is the formula for the variance of a Beta distribution. As $a$ and $b$ increases, the denominator will increase faster than the nominator. This means that if we use the Beta as a prior, the prior counts tell us something of how certain we are of our prior. The larger number of pseudo counts, the more certain we are.

$$
\operatorname{var}(X)=\frac{a b}{(a+b)^{2}+(a+b+1)}
$$

\section{Integrating out parameters}

With the Beta-Bernoulli and Beta-Binomial we can model one event or a series of events with the basic assumption that the events are independent and that the probability of success are the same in all of the events. But what if we would like to lift this last restriction, ie, we don't want to assume 
the same success probability for all events, how could we achieve this? To do this, we must get rid of the fixed $\theta$, this can be done by integrating out the $\theta$ parameter. It frees us from the restriction that the success probability has to be the same. It leaves us only with the assumption that it is drawn from a Beta distribution with some hyper-parameters (the $a$ and $b$ ).

To lift the restriction of a fixed $\theta$ we do something that is quite common in the Bayesian literature, we integrate out (also called marginalizing over) $\theta$. That is, we sum over all possible values of $\theta$.

In Equation 4.82 we had the expression for the Binomial distribution which tells us the probability of getting $k$ successes given $\theta$ and $N$. We then put a Beta prior on $\theta$ to get the posterior on $k$. But now we want to be independent of the fixed $\theta$, we do this by integrating out the $\theta$ from the Beta-Binomial model. The integrating out means that we integrate over the $\theta$ parameter in the Beta-Binomial model according to

$$
p(k \mid N, a, b)=\int_{0}^{1} \operatorname{Beta}(a, b) \operatorname{Bin}(N, \theta) d \theta
$$

Plugging in the respective formulas gives

$$
p(k \mid N, a, b)=\int_{0}^{1} \frac{\theta^{(a-1)}(1-\theta)^{(b-1)}}{B(a, b)}\left(\begin{array}{c}
N \\
k
\end{array}\right) \theta^{k}(1-\theta)^{N-k} d \theta
$$

Since $\theta$ is limited to the $0-1$ range, the integral is over this range. Extracting terms not depending on $\theta$

$$
p(k \mid N, a, b)=\left(\begin{array}{c}
N \\
k
\end{array}\right) \frac{1}{B(a, b)} \int_{0}^{1} \theta^{(a-1)}(1-\theta)^{(b-1)} \theta^{k}(1-\theta)^{N-k} d \theta
$$

Gather the exponents

$$
p(k \mid N, a, b)=\left(\begin{array}{l}
N \\
k
\end{array}\right) \frac{1}{B(a, b)} \int_{0}^{1} \theta^{(a+k-1)}(1-\theta)^{(b+N-k-1)} d \theta
$$

At this stage we have to recognize that the integral over $\theta$ is again a Beta function with the parameters $\mathrm{B}(\mathrm{a}+\mathrm{k}, \mathrm{b}+\mathrm{N}-\mathrm{k})$, so the final expression for our expression for the probability of $k$ independent of $\theta$, becomes

$$
p(k \mid N, a, b)=\left(\begin{array}{l}
N \\
k
\end{array}\right) \frac{B(a+k, b+N-k)}{B(a, b)}
$$

By integrating out the $\theta$ we make it "go away" and we can relax the assumption that the $\theta$ is fixed between the Bernoulli trials. Our only assumption left is that the (implicit) $\theta$ 's in each of the trials are drawn from a Beta distribution and that the trials are independent.

Integrating out a parameter in a model can sometimes be beneficial and sometimes not. In Paper III [35] we show that by not integrating out a parameter, we can implement an efficient and parallel implementation of LDA which 
is still mathematically correct. This is in contrast to other approaches which integrate out a parameter in the LDA model called $\phi$, making it impossible to parallelize the model and still be a correct model. In the LDA case where we model documents as a vector of counts of the words in the documents, the integrating out of the $\phi$ parameter makes each document dependent of every other document, thus making it impossible (or at least impractical) to parallelize. Since each document is dependent on the state of all the other documents, one would have to lock each thread and fetch the state of all documents being sampled on that thread to draw a sample on the current processor; this is not conducive to an efficient parallelization. This inexact approach to parallelizing LDA has become so common, that in several papers it is not even mentioned that the model is no longer an exact LDA model!

\section{Dirichlet-Multinomial}

In the preceding sections we have shown how the simple Bernoulli can be generalized to the Binomial distribution by having repeated trials. Here we show how one can extend the Binomial model to the more general case of several different outcomes rather than just binary outcomes. The extended model is called the multinomial model, it defines the Multinomial distribution. In the multinomial model we don't have just success or failure, but rather $N$ draws with one out of $K$ possible different outcomes called classes or categories. This is denoted ${ }^{3}$

$$
\boldsymbol{Y} \sim \operatorname{Multinomial}(N, \boldsymbol{\theta})
$$

Here both $\boldsymbol{Y}$ and $\boldsymbol{\theta}$ are $K$ dimensional vectors. $Y_{k}$ (the $k$ :th entry in $\boldsymbol{Y}$ ) is the class count of class $k$. In the Bernoulli and Binomial models $\theta$ expresses the probability of success. In the Multinomial model, $\boldsymbol{\theta}$ is a vector where each element of the vector expresses the probability of drawing that particular outcome class. So $\theta_{k}$ is a value between 0 and 1 which expresses the probability of drawing the $k$ :th outcome out of $K$ possible outcomes. In the multinomial model, the values in $\boldsymbol{\theta}$ must sum to 1. I.e., $\boldsymbol{\theta}$ is a probability distribution!

There are several special cases of the Multinomial distribution, this is natural since it is the generalization of several distributions. The special case of the Multinomial distribution where $N=1$, and $k=2$, is the Bernoulli distribution. When $k$ is 2 and $N>1$, it is the Binomial distribution, when $k>2$ and $N=1$, it is the so called categorical distribution. For example, if we have a 4-dimensional Multinomial distribution, one example draw from it could be $[0,0,1,0]$ which interpreted as a categorical draw would mean that the category we drew was category 3 (the vector is taken to be 1-indexed). A draw from a 4-dimensional $\operatorname{Multinomial}(7, \boldsymbol{\theta})$ could look like $[1,2,4,0]$, i.e. the resulting vector draw sums to 7 . In this one multinomial draw, category

\footnotetext{
${ }^{3}$ There are also other ways to encode the multinomial distribution
} 
1 was drawn once, category 2 , twice, category 3 , four times, and category 4 , zero times. We can see a draw from a Multinomial distribution as rolling a k-sided die $\mathrm{N}$ times and representing the counts of how many times each of sides comes up in a k-dimensional vector, where the k:th entry contains the number of times side $\mathrm{k}$ came up. The probability mass function for the multinomial is expressed in Equation 4.88

$$
p\left(Y_{1}, \ldots Y_{K} \mid \boldsymbol{\theta}\right)=\frac{N !}{y_{1} ! \ldots y_{K} !} \prod_{1}^{K} \theta_{k}^{y_{k}}
$$

The normalizing constant in the multinomial, like in the binomial case, compensates for the many different ways we can draw a particular combination of a multinomial outcome. As usual in the Bayesian paradigm we need a prior on the parameters. Since $\boldsymbol{\theta}$ is a probability distribution, we want a prior over probability distributions that is ideally conjugate to the multinomial. The multinomial is a generalization of the Binomial, and our prior will be a generalization of the Beta distribution. This conjugate prior is called the Dirichlet distribution and it is a prior over distributions.

$$
\theta \sim \operatorname{Dir}(\alpha)
$$

This means that a draw from the Dirichlet distribution, is itself a probability distribution! A draw from a Dirichlet distribution will look something like the discrete PMF in Figure 4.2. The Dirichlet distribution is parametrized by a vector of K-concentration parameters denoted $\boldsymbol{\alpha}$. The concentration parameters of the Dirichlet distribution decides what the probability distributions that the Dirichlet returns looks like. The $\boldsymbol{\alpha}$ parameter will determine how "peaked" the Dirichlet distribution will tend to be. The only requirement on the concentration parameters, is that they are $>0$ (i.e., they need not sum to one and does not have to be integers). The probability density function for the Dirichlet is

$$
p\left(\theta_{1}, \ldots \theta_{K} \mid \boldsymbol{\alpha}\right)=\frac{1}{B(\boldsymbol{\alpha})} \prod_{1}^{K} \theta_{k}^{\alpha_{k}-1}
$$

Here $B(\boldsymbol{\alpha})$ is the multivariate Beta function (remember that we had the Beta function in the Binomial case), this function is usually expressed in terms of the Gamma function, which turns Equation 4.90 into

$$
p\left(\theta_{1}, \ldots \theta_{K} \mid \boldsymbol{\alpha}\right)=\frac{\Gamma\left(\sum_{1}^{K} \alpha_{k}\right)}{\prod_{1}^{K} \Gamma\left(\alpha_{k}\right)} \prod_{1}^{K} \theta_{k}^{\alpha_{k}-1}
$$

Where $\Gamma(\bullet)$ is the Gamma function, which is a generalization of the faculty (!) function to non-integer values. If $g$ is a positive integer, then $\operatorname{Gamma}(g)=$ 
$(g-1)$ !. If we combine the Dirichlet prior and the multinomial likelihood we get an expression for the posterior

$$
p\left(\boldsymbol{\theta} \mid Y_{1}, . . Y_{K}, \boldsymbol{\alpha}\right) \propto \operatorname{Dir}(\boldsymbol{\theta} \mid \boldsymbol{\alpha}) \text { Multinomial }(N, \boldsymbol{\theta})
$$

Which, when we use our old trick of ignoring normalizing constants, expands to

$$
p\left(\theta_{1}, \ldots \theta_{K} \mid Y_{1}, . . Y_{K}, \boldsymbol{\alpha}\right) \propto \prod_{1}^{K} \theta_{k}^{\alpha_{k}-1} \prod_{1}^{K} \theta_{k}^{y_{k}}
$$

Gather the exponents

$$
p\left(\theta_{1}, \ldots \theta_{K} \mid Y_{1}, . . Y_{K}, \boldsymbol{\alpha}\right) \propto \prod_{1}^{K} \theta_{k}^{y_{k}+\alpha_{k}-1}
$$

Where $y_{k}$ is the number of observed values of category $k$ in the dataset. By again studying the form of Equation 4.94 we see that it is again a Dirichlet with parameters $Y_{k}+\alpha_{k}$. Putting back the normalization constants we get the full posterior.

$$
p\left(\theta_{1}, \ldots \theta_{K} \mid Y_{1}, . . Y_{K}, \boldsymbol{\alpha}\right)=\frac{1}{B(\boldsymbol{Y}+\boldsymbol{\alpha})} \prod_{1}^{K} \theta_{k}^{y_{k}+\alpha_{k}-1}
$$

The update rule here then consists of updating the prior Dirichlet hyperparameters with the number of counts of the respective categories seen in the data. So the posterior on $\boldsymbol{\theta}$ becomes:

$$
\boldsymbol{\theta} \sim \operatorname{Dir}(\boldsymbol{\alpha}+\boldsymbol{Y})
$$

\section{Dirichlet-Categorical}

As mentioned in Section 4.4, the categorical distribution can be seen as a special case of the multinomial distribution where $N=1$. The categorical distribution, like the multinomial is parametrized by a $K$ dimensional vector $\boldsymbol{\theta}$ with the exact same interpretation as for the multinomial model. One specific fact to note is that since in the categorical case we only do one draw, we don't need the normalizing constant of the multinomial which compensates for the many different ways to generate a particular outcome. If $Y$ has a categorical distribution it is denoted

$$
Y \sim \operatorname{Cat}(\boldsymbol{\theta}, K)
$$

Or simply

$$
Y \sim \operatorname{Cat}(\boldsymbol{\theta})
$$


Observe that in the categorical situation, the meaning of $Y$ is different than in the multinomial case. In the categorical case, $Y$ is a scalar, and $Y=k$ means that $Y$ has class $k$. In the multinomial case, $Y$ is a vector, and $Y_{k}$ is the number of times class $k$ occurred. What can be confusing is that sometimes the categorical outcome of $Y$ is equivalently encoded as a vector with the $k$ :th entry set to one (i.e. a one draw from a $k$-dimensional multinomial), often called a one-of- $k$ encoding or one-hot encoding.

If we do $N$ categorical draws and sum the class occurrences in a vector we get back the multinomial. If we see the categorical distribution as one draw from the multinomial distribution, the pmf for the categorical distribution becomes extremely simple. The probability of a particular outcome is defined by $\boldsymbol{\theta}$

$$
p(Y=k)=\theta_{k}
$$

Putting a Dirichlet prior on $\theta$ and using the same proof as for the DirichletMultinomial we can show that the posterior on $\theta$ is again a Dirichlet:

$$
\boldsymbol{\theta} \sim \operatorname{Dir}\left(\boldsymbol{\alpha}+\boldsymbol{C}_{\boldsymbol{c}}\right)
$$

Where $\boldsymbol{C}_{\boldsymbol{c}}$ is the vector of the number of observed counts per class summed over all observations. That is, if we start with a Dirichlet prior with hyperparameters $\boldsymbol{\alpha}$ and combine it with a vector of counts $\boldsymbol{C}_{\boldsymbol{c}}$ of draws coming from a Categorical distribution, then the posterior is again a Dirichlet distribution with parameters $\boldsymbol{\alpha}+\boldsymbol{C}_{\boldsymbol{c}}$. If we represent the categorical distribution as a multinomial with just one draw, the counts for one observation will be a vector with all zeros except for a one in the observed category. We then sum over all observations to get the full $\boldsymbol{C}_{\boldsymbol{c}}$ vector of counts.

We can see the Multinomial as a "batch" execution of many Categorical draws, where the multinomial $\boldsymbol{Y}$ vector contains the sums of the many categorical draws.

\section{Bayesian Linear Regression}

After this general introduction to Bayesian Learning, let us look at an example how we can apply the Bayesian method of learning. For familiarity, and due to its importance, we shall return to linear regression also in the Bayesian context and see how we can cast it as a Bayesian learning problem. In the Bayesian framework, everything is expressed in terms of probabilities. In our exposition of the ordinary linear regression we did not have an explicit notion of probability, but here we shall. Recall that one of the advantages of the Bayesian framework is that we get probability distributions over all of our parameter estimates. So how do we recast our linear regression

$$
\boldsymbol{y}=\boldsymbol{X} \boldsymbol{w}+\sigma
$$


in a way that we get a probability distribution? The strategy will be to let the linear relation model the mean of $y$ instead of $y$ directly. Expressed in the language of probability, this turns into:

$$
p(\boldsymbol{y} \mid \boldsymbol{X}, \boldsymbol{w}, \sigma)=\mathcal{N}\left(\boldsymbol{X} \boldsymbol{w}, \sigma^{2} \boldsymbol{I}\right)
$$

Which denotes that $\boldsymbol{y}$ has a multivariate normal distribution, the extension of the normal distribution to many dimensions. The expression for the multivariate normal pdf is:

$$
p(\boldsymbol{y} \mid \boldsymbol{X}, \boldsymbol{w}, \sigma)=\frac{1}{(2 \pi)^{-\frac{p}{2}}} \frac{1}{\operatorname{det}(\sigma \boldsymbol{I})^{-\frac{1}{2}}} \exp \left(-\frac{1}{2}(\boldsymbol{y}-\boldsymbol{X} \boldsymbol{w})^{T}\left(\sigma^{2} \boldsymbol{I}\right)^{-1}(\boldsymbol{y}-\boldsymbol{X} \boldsymbol{w})\right)
$$

Where $\boldsymbol{y}$ is still a vector of observations and $\boldsymbol{X}$ is the corresponding matrix of observed variables and $\boldsymbol{w}$ is the vector of weights, one for each of the columns in $\boldsymbol{X} . \boldsymbol{I}$ is the identity matrix, that is, a square matrix with 1's on the diagonal and zeros everywhere else. $p$ is the number of columns in $\boldsymbol{X}$. This particular form encodes the assumption that the errors are independent. This formula should be read as, "Given that we know $\boldsymbol{X}, \boldsymbol{w}$ and $\sigma, \boldsymbol{y}$ is normally distributed with mean $\boldsymbol{X} \boldsymbol{w}$ and standard deviation $\sigma$ ". Here, the normal distribution is extended to the multivariate case, where instead of modeling one scalar, it models a random vector in which each entry is normally distributed. To simplify the presentation we will assume that $\sigma$ is known, which in general it is not, but the analysis can be extended to include $\sigma$ too.

As in the linear regression case we are interested in learning the value of the parameters $\boldsymbol{w}$. So we are really interested in:

$$
p(\boldsymbol{w} \mid \boldsymbol{X}, \boldsymbol{y}, \sigma)
$$

that is, the probability of the parameters given the data. Remember that in the Bayesian paradigm we always start with a prior probability on our parameters, we then observe some data and the update our probability to arrive at the posterior probability distribution on our parameters. We denote this prior with $p(\boldsymbol{w})$ which is the prior probability distribution we place over the parameters $\boldsymbol{w}$. In this particular case we put a zero centered normal prior distribution on $\boldsymbol{w}$ which in general is a reasonable assumption.

$$
p(\boldsymbol{w})=\mathcal{N}\left(\mathbf{0}, \sigma_{0}^{2} \boldsymbol{I}\right)
$$

In Paper $\mathrm{V}$ we use a different prior, called the Horseshoe on our parameter vector. The Horseshoe puts a stronger prior on $\boldsymbol{w}$ and expresses the prior belief, that the entries in $\boldsymbol{w}$ are either very close to zero or large. 
With these preliminaries taken care of, we shall again return to our data in Table 4.1. We continue to use the matrix notation to specify our problem. In the linear regression case we had the linear equation

$$
\boldsymbol{y}=\boldsymbol{X} \boldsymbol{w}+\sigma
$$

Where we now have adopted the notational convention that we drop the explicit $*$ symbol with the understanding that $\boldsymbol{X} \boldsymbol{w}$ means $\boldsymbol{X} * \boldsymbol{w}$. We have shown that the Bayesian equation has the general form

$$
p(A \mid B)=\frac{p(B \mid A) p(A)}{p(B)}
$$

In our case $A$ corresponds to the weight vector $\boldsymbol{w}$. $B$ corresponds to our data, $\boldsymbol{X}$ and $\boldsymbol{y}$, and the fixed $\sigma$. By plugging in these variables, our formulation becomes

$$
p(\boldsymbol{w} \mid \boldsymbol{X}, \boldsymbol{y}, \sigma)=\frac{p(\boldsymbol{y}, \boldsymbol{X}, \sigma \mid \boldsymbol{w}) p(\boldsymbol{w})}{p(\boldsymbol{X}, \boldsymbol{y}, \sigma)}
$$

Which should be read as: "What is the probability distribution over $\boldsymbol{w}$ given (our data) $\boldsymbol{X}$, and $\boldsymbol{y}$ (and $\sigma$ )". In 4.108 we have used the standard Bayes formula to find $A \mid B$ when we know $B \mid A$, but the expression $p(\boldsymbol{y}, \boldsymbol{X}, \sigma \mid \boldsymbol{w})$ (corresponding to $B \mid A$ in the Bayes Rule) is not really something that we have available. We would much rather have the expression in 4.102, since that is the way we model how the data is generated, given the parameters. Using the rules of probability exemplified in 4.65 we can go from $p(\boldsymbol{y}, \boldsymbol{X}, \sigma \mid \boldsymbol{w}) p(\boldsymbol{w})$ to $p(\boldsymbol{y} \mid \boldsymbol{X}, \sigma, \boldsymbol{w}) p(\boldsymbol{w})$ as follows:

$$
\begin{aligned}
p(\boldsymbol{y}, \boldsymbol{X}, \sigma \mid \boldsymbol{w}) p(\boldsymbol{w}) & =p(\boldsymbol{y}, \boldsymbol{X} \mid \sigma, \boldsymbol{w}) p(\sigma, \boldsymbol{w}) \\
& =p(\boldsymbol{y} \mid \boldsymbol{X}, \sigma, \boldsymbol{w}) p(\boldsymbol{X}, \sigma, \boldsymbol{w})
\end{aligned}
$$

Since $\boldsymbol{X}$ are observed data and $\sigma$ is given, the expression $p(\boldsymbol{X}, \sigma, \boldsymbol{w})$ reduces again to $p(\boldsymbol{w})$. Which leaves us with

$$
p(\boldsymbol{y}, \boldsymbol{X}, \sigma \mid \boldsymbol{w}) p(\boldsymbol{w})=p(\boldsymbol{y} \mid \boldsymbol{X}, \sigma, \boldsymbol{w}) p(\boldsymbol{w})
$$

Substituting into 4.108 leaves us with:

$$
p(\boldsymbol{w} \mid \boldsymbol{X}, \boldsymbol{y}, \sigma)=\frac{p(\boldsymbol{y} \mid \boldsymbol{X}, \sigma, \boldsymbol{w}) p(\boldsymbol{w})}{p(\boldsymbol{X}, \boldsymbol{y}, \sigma)}
$$

Once again we will employ our trick of ignoring the normalizing denominator, giving us:

$$
p(\boldsymbol{w} \mid \boldsymbol{X}, \boldsymbol{y}, \sigma) \propto p(\boldsymbol{y} \mid \boldsymbol{X}, \sigma, \boldsymbol{w}) p(\boldsymbol{w})
$$


In the next step, we need is to substitute concrete expressions for the probabilities involved, i.e $p(\boldsymbol{y} \mid \boldsymbol{X}, \sigma, \boldsymbol{w})$ and $p(\boldsymbol{w})$. From our modeling assumption in 4.103, we have assumed that $p(\boldsymbol{y} \mid \boldsymbol{X}, \sigma, \boldsymbol{w})$ is a normal distribution. In 4.105 we assumed a normal prior for $p(\boldsymbol{w})$.

It would require too much multivariate analysis and Bayesian theory to go into the details of the motivations for the various expressions so we will summarize the results here. For a detailed expositions we refer to Gelman, Carlin, Stern, and Rubin [36] and Jaynes [37].

The mathematical forms of the likelihood and the prior defines the mathematical model. If we are careful in our selection of the form for the likelihood and prior we can again get a conjugate model. Remember that conjugate means that the posterior have the same mathematical form as the prior. This is very useful if we can choose a prior of a known distribution from which it is easy to draw random samples. Examples of some widely used conjugate models are the Bernoulli-Beta, Binomial-Beta, Poisson-Gamma, MultinomialDirichlet and Normal-Normal models, some of which we discuss in Section 4.4.

In this Bayesian linear regression example we will use a Normal-Normal model, in our next example Bayesian Naive Bayes Classification (in Section 4.4) we will use the Multinomial-Dirichlet model. Both of these models are used in our papers. To get a Normal-Normal conjugate model we select a Normal model for the likelihood function and a normal prior for the weight vector $\boldsymbol{w}$. This then leads to the following Bayesian model specification:

$$
\begin{gathered}
p(\boldsymbol{y} \mid \boldsymbol{X}, \sigma, \boldsymbol{w}) \sim \mathcal{N}\left(\boldsymbol{X} \boldsymbol{w}, \sigma^{2} \boldsymbol{I}\right) \\
p(\boldsymbol{w}) \sim \mathcal{N}\left(\mathbf{0}, \sigma_{0}^{2} \boldsymbol{I}\right)
\end{gathered}
$$

Plugging in the specific expressions for 4.114 and 4.115 gives us:

$$
p(\boldsymbol{w} \mid \boldsymbol{X}, \boldsymbol{y}, \sigma) \propto \mathcal{N}\left(\boldsymbol{X} \boldsymbol{w}, \sigma^{2} \boldsymbol{I}\right) \mathcal{N}\left(\mathbf{0}, \sigma_{0}^{2} \boldsymbol{I}\right)
$$

For the normal distributions there are several known results for products of normals, but below we will give a simplified derivation of the main result. Remember that everything is in vector/matrix notation, so $\boldsymbol{X}$ is a matrix with all the observed $x$ 's. The matrix has dimension; number of rows n number of columns, where the columns is the number of features plus one column of 1 's for the intercept. $\boldsymbol{y}$ is a column vector which is $N$ long and $\boldsymbol{w}$ is a row vector which is $p$ long, the number of input features +1 for the intercept. Since we use the proportional form, we remove all constants and end up with the following expression for the posterior on $\boldsymbol{w}$ : 


$$
\begin{gathered}
p(\boldsymbol{w} \mid \boldsymbol{X}, \boldsymbol{y}, \sigma) \propto \underbrace{\exp \left(-\frac{1}{2}(\boldsymbol{y}-\boldsymbol{X} \boldsymbol{w})^{T}\left(\sigma^{2} \boldsymbol{I}\right)^{-1}(\boldsymbol{y}-\boldsymbol{X} \boldsymbol{w})\right)}_{\text {normal likelihood from } 4.114} \\
\underbrace{\exp \left(-\frac{1}{2}(\boldsymbol{w}-\mathbf{0})^{T}\left(\sigma_{0}^{2} \boldsymbol{I}\right)^{-1}(\boldsymbol{w}-\mathbf{0})\right)}_{\text {normal prior from } 4.115}
\end{gathered}
$$

Extract $\sigma^{\prime} s,-\mathbf{0}$ goes away:

$$
\begin{gathered}
p(\boldsymbol{w} \mid \boldsymbol{X}, \boldsymbol{y}, \sigma) \propto \quad \exp \left(-\frac{1}{2 \sigma^{2}}(\boldsymbol{y}-\boldsymbol{X} \boldsymbol{w})^{T} \boldsymbol{I}^{-1}(\boldsymbol{y}-\boldsymbol{X} \boldsymbol{w})\right) \\
\exp \left(-\frac{1}{2 \sigma_{0}^{2}} \boldsymbol{w}^{T} \boldsymbol{I}^{-1} \boldsymbol{w}\right)
\end{gathered}
$$

Identity matrix goes away (the inverse of the identity matrix is the identity matrix):

$$
p(\boldsymbol{w} \mid \boldsymbol{X}, \boldsymbol{y}, \sigma) \propto \exp \left(-\frac{1}{2 \sigma^{2}}(\boldsymbol{y}-\boldsymbol{X} \boldsymbol{w})^{T}(\boldsymbol{y}-\boldsymbol{X} \boldsymbol{w})\right) \exp \left(-\frac{1}{2 \sigma_{0}^{2}} \boldsymbol{w}^{T} \boldsymbol{w}\right)
$$

The exponents of products of exponentials sum:

$$
p(\boldsymbol{w} \mid \boldsymbol{X}, \boldsymbol{y}, \sigma) \propto \exp \left[-\frac{1}{2 \sigma^{2}}(\boldsymbol{y}-\boldsymbol{X} \boldsymbol{w})^{T}(\boldsymbol{y}-\boldsymbol{X} \boldsymbol{w})-\frac{1}{2 \sigma_{0}^{2}} \boldsymbol{w}^{T} \boldsymbol{w}\right]
$$

Extract $-1 / 2$ and let $1 / \sigma^{2}=a$ and $1 / \sigma_{0}^{2}=b$

$$
p(\boldsymbol{w} \mid \boldsymbol{X}, \boldsymbol{y}, \sigma) \propto \exp \left[-\frac{1}{2}\left(a(\boldsymbol{y}-\boldsymbol{X} \boldsymbol{w})^{T}(\boldsymbol{y}-\boldsymbol{X} \boldsymbol{w})+b \boldsymbol{w}^{T} \boldsymbol{w}\right)\right]
$$

Cross multiply:

$$
p(\boldsymbol{w} \mid \boldsymbol{X}, \boldsymbol{y}, \sigma) \propto \exp \left[-\frac{1}{2} a\left(\boldsymbol{y}^{T} \boldsymbol{y}-(\boldsymbol{X} \boldsymbol{w})^{T} \boldsymbol{y}-\boldsymbol{y}^{T}(\boldsymbol{X} \boldsymbol{w})-(\boldsymbol{X} \boldsymbol{w})^{T} \boldsymbol{X} \boldsymbol{w}\right)+b \boldsymbol{w}^{T} \boldsymbol{w}\right]
$$

Use rules for transpose to get terms in same form. $(\boldsymbol{X} \boldsymbol{w})^{T}=\boldsymbol{w}^{T} \boldsymbol{X}^{T}$ so $(\boldsymbol{X} \boldsymbol{w})^{T} \boldsymbol{y}=\boldsymbol{w}^{T} \boldsymbol{X}^{T} \boldsymbol{y} \boldsymbol{y}^{T}(\boldsymbol{X} \boldsymbol{w})=(\boldsymbol{X} \boldsymbol{w})^{T} \boldsymbol{y}=\boldsymbol{w}^{T} \boldsymbol{X}^{T} \boldsymbol{y}:$

$$
p(\boldsymbol{w} \mid \boldsymbol{X}, \boldsymbol{y}, \sigma) \propto \exp \left[-\frac{1}{2} a\left(\boldsymbol{y}^{T} \boldsymbol{y}-2 \boldsymbol{w}^{T} \boldsymbol{X}^{T} \boldsymbol{y}-\boldsymbol{w}^{T} \boldsymbol{X}^{T} \boldsymbol{X} \boldsymbol{w}\right)+b \boldsymbol{w}^{T} \boldsymbol{w}\right]
$$

Distribute $a$ :

$$
p(\boldsymbol{w} \mid \boldsymbol{X}, \boldsymbol{y}, \sigma) \propto \exp \left[-\frac{1}{2} a \boldsymbol{y}^{T} \boldsymbol{y}-2 a \boldsymbol{w}^{T} \boldsymbol{X}^{T} \boldsymbol{y}-\boldsymbol{w}^{T} a \boldsymbol{X}^{T} \boldsymbol{X} \boldsymbol{w}+b \boldsymbol{w}^{T} \boldsymbol{w}\right]
$$


Simplify last two terms. $b \boldsymbol{w}^{T} \boldsymbol{w}=\boldsymbol{w}^{T} b \boldsymbol{I} \boldsymbol{w}$.

$$
p(\boldsymbol{w} \mid \boldsymbol{X}, \boldsymbol{y}, \sigma) \propto \exp \left[-\frac{1}{2} a \boldsymbol{y}^{T} \boldsymbol{y}-2 a \boldsymbol{w}^{T} \boldsymbol{X}^{T} \boldsymbol{y}-\boldsymbol{w}^{T}\left(a \boldsymbol{X}^{T} \boldsymbol{X}+b \boldsymbol{I}\right) \boldsymbol{w}\right]
$$

Completing the square gives:

$$
p(\boldsymbol{w} \mid \boldsymbol{X}, \boldsymbol{y}, \sigma) \propto \exp \left[-\frac{1}{2}\left(\boldsymbol{w}-\boldsymbol{\mu}_{\boldsymbol{n}}\right)^{T} \boldsymbol{\Lambda}^{-1}\left(\boldsymbol{w}-\boldsymbol{\mu}_{\boldsymbol{n}}\right)\right]
$$

Where $\boldsymbol{\Lambda}$ is:

$$
\boldsymbol{\Lambda}=a \boldsymbol{X}^{T} \boldsymbol{X}+b \boldsymbol{I}
$$

and $\boldsymbol{\mu}_{n}$ :

$$
\boldsymbol{\mu}_{\boldsymbol{n}}=a \boldsymbol{\Lambda}^{-1} \boldsymbol{X}^{T} \boldsymbol{y}=a\left(a \boldsymbol{X}^{T} \boldsymbol{X}+b \boldsymbol{I}\right)^{-1} \boldsymbol{X}^{T} \boldsymbol{y}
$$

If we pick apart Equation 4.122 we can see that it is a multivariate normal with variance $\boldsymbol{\Lambda}^{-1}$ and mean $\boldsymbol{\mu}_{\boldsymbol{n}}$. Expressed in probabilistic notation, the posterior for $\boldsymbol{w}$ is:

$$
p(\boldsymbol{w} \mid \boldsymbol{X}, \boldsymbol{y}, \sigma) \sim \mathcal{N}\left(\boldsymbol{\mu}_{\boldsymbol{n}}, \boldsymbol{\Lambda}^{-1}\right)
$$

At this point we can compare with the result for the standard linear regression that we arrived to in 4.48 which is repeated here (with * removed) for convenience:

$$
\boldsymbol{w}=\left(\boldsymbol{X}^{T} \boldsymbol{X}\right)^{-1} \boldsymbol{X}^{T} \boldsymbol{y}
$$

We see that the expression for the mean $\left(\boldsymbol{\mu}_{\boldsymbol{n}}\right)$ in the Bayesian formulation is similar as the standard OLS result. In the Bayesian result the mean contains both the error term $a$, which is $1 / \sigma^{2}$, the variance of the data, and the prior

\section{Completing the Matrix Square}

There is an analog matrix version of completing the square. We can go from:

$$
\boldsymbol{y}^{T} \boldsymbol{A} \boldsymbol{y}-(\boldsymbol{X} \boldsymbol{w})^{T} \boldsymbol{A} \boldsymbol{y}-\boldsymbol{y}^{T} \boldsymbol{A} \boldsymbol{X} \boldsymbol{w}+\boldsymbol{w}^{T} \boldsymbol{X}^{T} \boldsymbol{A} \boldsymbol{X} \boldsymbol{w}
$$

to

$$
(\boldsymbol{y}-\boldsymbol{X} \boldsymbol{w})^{T} \boldsymbol{A}(\boldsymbol{y}-\boldsymbol{X} \boldsymbol{w})
$$

We use a version of this identity in Equation 4.121. 
uncertainty $b=1 / \sigma_{0}^{2}$. This is the same result one gets when using, so called, ridge regression in standard OLS. So putting a normal prior on the betas in the Bayesian formulation can be interpreted as using ridge regression. Other types of priors on the betas can be used and they sometimes lead to other types of OLS regularization schemes, but there are Bayesian priors that have no standard OLS correspondence. In this particular example we chose a normalnormal model which gives a closed form expression for the posterior. But if we use the flexibility of the Bayesian approach and select some other prior we are not (in fact, most often not) guaranteed to get a closed form expression for the posterior and then we need to use some version of MCMC to do inference in the model.

\section{Bayesian Naive Bayes Classification}

In Section 4.3 we discussed the standard Naive Bayes classification technique. Despite its name, the Naive Bayes approach, is not Bayesian in the truest sense since there are no clearly defined priors on the parameters. Here we will discuss how the Naive Bayes approach, can be re-cast as a truly Bayesian technique.

\section{Model Specification}

The first thing we need in building our Bayesian classification model, is just that, a model. Specifically a probability model of our problem. The second step is to put priors on the parameters that control the different probability distributions involved. Below we sketch this process (see also Murphy [30, Ch. 3]). We start with what we are interested in:

$$
p(Y \mid \boldsymbol{X}, \text { Data })
$$

I.e, we are interested in calculating the probability distribution of $Y$, i.e. the class for a new unknown data point encoded in $\boldsymbol{X}$, given some historical Data. Our model will be a so called parametric model, i.e.. we have some parameters that control the model behavior. We don't know what these parameters are at this moment, but we introduce them into the model. We denote these parameters $\boldsymbol{\theta}$, the model now becomes

$$
p(Y \mid \boldsymbol{X}, \boldsymbol{D a t a}, \boldsymbol{\theta})
$$

$\boldsymbol{X}$ is a random vector consisting of input variables or features. In this example we will use capitals to denote the new input $\boldsymbol{X}$, and the corresponding new unknown class $Y$ that we want to predict. Observations of historical $\boldsymbol{X}$ and $Y$, will be denoted with lowercase $x$ and $y$. An observation of an RV is called an outcome. So the Data is a set of $\mathrm{N}$ observations of outcomes of historical $\boldsymbol{X}$ and $Y$ : 


\begin{tabular}{|l|l|l|l|l|l|l|l|}
\hline \multicolumn{7}{|c|}{ Bug Database } \\
\hline Bug Id & Site & Customer & Priority & Heading & Description & Component \\
\hline \hline BugId-01 & Site 1 & Customer 1 & Priority 3 & No access & $\begin{array}{l}\text { Cannot access } \\
\text { the database... }\end{array}$ & Database \\
\hline BugId-02 & Site 2 & Customer 4 & Priority 1 & No sync & $\begin{array}{l}\text { Sync is lost be- } \\
\text { tween... }\end{array}$ & Controller \\
\hline BugId-4709 & Site 1 & Customer 12 & Priority 1 & $\begin{array}{l}\text { Wrong } \\
\text { contract }\end{array}$ & $\begin{array}{l}\text { Wrong con- } \\
\text { tract is re- } \\
\text { turned... }\end{array}$ & $\begin{array}{l}\text { Business } \\
\text { logic }\end{array}$ \\
\hline BugId-4710 & Site 4 & Customer 3 & Priority 3 & $\begin{array}{l}\text { Missing } \\
\text { menu }\end{array}$ & $\begin{array}{l}\text { Menu not visi- } \\
\text { ble... }\end{array}$ & UI \\
\hline BugId-4711 & Site 5 & Customer 1 & Priority 2 & Backup & Replication ... & Database \\
\hline
\end{tabular}

Table 4.6: A simplified imaginary database of bug reports. Ellipsis at the end of descriptions symbolizes that a longer text follows.

$$
\operatorname{Data}=\left(\left(\boldsymbol{x}_{1}, y_{1}\right), \ldots,\left(\boldsymbol{x}_{\boldsymbol{N}}, y_{N}\right)\right)
$$

The $y^{\prime} s$ are integer value observations of the $\operatorname{RV} Y$, where the integer value denotes the category index of the outcome state. The $\boldsymbol{x}^{\prime} s$ are vectors of observations of outcomes of the random vector $\boldsymbol{X}$. Each $\boldsymbol{x}$ vector consists of the outcome of each one of the categorical input variables in the historical data. So for instance $x_{1,1}$ is the outcome of the Customer variable of the 1:st observation which can take on values in the set (Customer1, Customer $2, \ldots$, Customer12). Each of the inputs in $\boldsymbol{X}$ is a nominal variable (in our case we only use nominal variables but other types can also be modeled) and can have different numbers of output states, so the Site variable for instance, have fewer possible states (Site1,...Site5). For convenience, we repeat Table 4.6, the example table of outcomes that we used in the Naive Bayes example in Section 4.3.

The different numbers of possible outcomes for the different variables gives us some notational challenges. To clarify, small $x_{i, p}$ is the specific observed outcome of the $p$ :th variable in $\boldsymbol{X}$ for the $i$ :th observation, while capital $X_{p}$ is the $p:$ th random variable in an unobserved $\boldsymbol{X}$.

As in the Naive Bayes example we will ignore the textual variables and refer to the papers in the thesis for discussions of techniques for how to represent the textual parts of the bug reports. For this example then, the classification data $\boldsymbol{X}$ will consist of three (ignoring the text fields) categorical variables, Customer, Site and Priority. So an example of an observed data point (BugId4710) is:

$x_{i}=($ Customer $=$ Customer 3, Site $=$ Site 4 , Priority $=3) \rightarrow y=$ $($ Component $=U I)$ 
$x_{i, 2}$ denotes the specific outcome (Site 4 in this case) of the 2:nd variable (the Site variable) in the $i$ :th observation. Our model assumes the data points are independent if we know the parameters $(\boldsymbol{\theta})$ of the model, i.e. one observation have no impact on the next. This is a stronger assumption than what is commonly necessary in the Bayesian paradigm. In the Bayesian approach, the common assumption is that the data is exchangeable. Exchangeable just means that we can permute the order of the observations without violating any assumptions of the data generating process.

A difference in this example from the previous examples is that here we are interested in the posterior predictive distribution of a new data point rather than the parameters of the model. That is, we are not interested in studying the posterior distribution of the parameters $\boldsymbol{\theta}$, but only in predicting the class of a new $Y$ given a new $\boldsymbol{X}$. By the definition of conditional probability

$$
p(Y \mid \boldsymbol{X}, \text { Data }, \boldsymbol{\theta})=\frac{p(Y, \boldsymbol{X}, \boldsymbol{D a t a}, \boldsymbol{\theta})}{p(\boldsymbol{X}, \text { Data }, \boldsymbol{\theta})}
$$

But the expression on the right hand side here is not really something that we can work with directly. We would like to separate the new data points $\boldsymbol{X}$ and $Y$ from the Data and $\boldsymbol{\theta}$. We can do that by the following derivation. First, the denominator does not depend on $Y$ so we can drop it.

$$
p(Y \mid \boldsymbol{X}, \boldsymbol{D a t a}, \boldsymbol{\theta}) \propto p(Y, \boldsymbol{X}, \boldsymbol{D a t a}, \boldsymbol{\theta})
$$

We can then by the chain rule reformulate the joint probability on the right hand to

$$
p(Y \mid \boldsymbol{X}, \boldsymbol{D a t a}, \boldsymbol{\theta}) \propto p(Y, \boldsymbol{X}, \boldsymbol{D a t a} \mid \boldsymbol{\theta}) p(\boldsymbol{\theta})
$$

A very powerful tool in the Bayesian paradigm is thinking about independence relations between the constituents in the model. The underlying assumption is that given the model parameters, some process is generating our data points. The independence assumption in Equation 4.127 is that the historical Data and, $\boldsymbol{X}$ and $Y$, are independent given the model parameters, which gives us:

$$
p(Y \mid \boldsymbol{X}, \boldsymbol{D a t a}, \boldsymbol{\theta}) \propto p(Y, \boldsymbol{X} \mid \boldsymbol{\theta}) p(\boldsymbol{D a t a} \mid \boldsymbol{\theta}) p(\boldsymbol{\theta})
$$

Further, $p(Y, \boldsymbol{X} \mid \boldsymbol{\theta})$ can by the chain rule be factored as $p(\boldsymbol{X} \mid Y, \boldsymbol{\theta}) p(Y \mid \boldsymbol{\theta})$ which leaves us with:

$$
p(Y \mid \boldsymbol{X}, \boldsymbol{D a t a}, \boldsymbol{\theta}) \propto p(\boldsymbol{X} \mid Y, \boldsymbol{\theta}) p(Y \mid \boldsymbol{\theta}) p(\text { Data } \mid \boldsymbol{\theta}) p(\boldsymbol{\theta})
$$

To complete our Bayesian model we also need to discuss the model parameters $\boldsymbol{\theta}$, which is a vector with all the parameters that we need. As usual, we put a prior on those parameters which is the $p(\boldsymbol{\theta}) . \boldsymbol{\theta}$ is a vector of parameters that will control the concrete probability distributions that we put on our variables of interest. $\boldsymbol{\theta}$ consists of two sets of priors; the priors on $Y$ (i.e. 
the class labels) and the priors on $\boldsymbol{X} \mid Y$ (i.e. the probability of a variable taking a specific value given the class label). Now, if we are only interested in predictions of $Y$ and not inherently interested in analyzing $\boldsymbol{\theta}$, we can integrate out $\boldsymbol{\theta}$ (as discussed in Section 4.4).

$$
p(Y \mid \boldsymbol{X}, \boldsymbol{D a t a}) \propto \int p(\boldsymbol{X} \mid Y, \boldsymbol{\theta}) p(Y \mid \boldsymbol{\theta}) p(\text { Data } \mid \boldsymbol{\theta}) p(\boldsymbol{\theta}) d \boldsymbol{\theta}
$$

This can seem like an unnecessary step, we first introduce the parameters $\boldsymbol{\theta}$ and then we immediately integrate them out. The parameters in the model acts as a "bridge" between the historical data and the prediction of a new observation. This is a common approach not only in the Bayesian paradigm. The $\boldsymbol{\theta}$ 's represent the unseen (sometimes called latent) parts of the probabilistic model. The model together with the parameters describe what we imagine is the underlying mechanism for generating the observations we have been given. The latent parts are needed for inference in the model, but we are not always interested in them, like in this case where we are only interested in predicting a new $Y$. When we are not directly interested in the latent parameters we integrate them out as in Equation 4.128.

Worth noting here is a notational shortcut. What looks like a single integral in 4.128 is really many integrals. It consists of the integral over the priors over the $Y$ 's and the integrals over every variable in the model and their priors.

We split $\boldsymbol{\theta}$ into two parts, one part controls the probability distribution over the component (class) probabilities (Controller, UI, etc.) which we call $\boldsymbol{\pi}$. The second part controls the conditional probabilities of the variables given the component $(\mathrm{p}($ Site $=$ Site $4 \mid$ Component=Controller $))$ which we call $\phi$. So our full vector of parameters is $\boldsymbol{\theta}=(\boldsymbol{\pi}, \phi)$. I.e., the priors on the classes and the priors on the set of outcomes given the class labels for each variable in our model. In this case we will use a so called factored prior for $\boldsymbol{\theta}$, i.e. the different parts of the prior are independent of each other and thus can be factored according to $p(\boldsymbol{\pi}, \boldsymbol{\phi})=p(\boldsymbol{\pi}) p(\boldsymbol{\phi})$. In the original Naive Bayes version the parameters are calculated directly from the data. But in the fully Bayesian approach we need priors on the parameters. Below we describe which probability distributions we choose as priors for the different parts of our model.

\section{Probability Distributions on $Y$}

The probability distribution over the components/classes we model using a categorical distribution, since the components are categorical in nature. Remember, an RV which is distributed according to a categorical distribution takes one categorical value from a set of outcome classes. In our case, the set for $Y$ consists of the components in the dataset, i.e. \{UI, Database, Controller, Business Logic $\}$. So a draw from the categorical distribution will return one 
of the outcomes in that set. We can express that component is categorically distributed as follows:

$$
\text { Component } \sim \operatorname{Cat}(K, \boldsymbol{\pi})
$$

where $\operatorname{Cat}(K, \boldsymbol{\pi})$ means that Component is distributed according to a Categorical distribution. The Categorical distribution is parametrized with $K$ which is the number of categories and $\boldsymbol{\pi}$ which is a K-dimensional vector with probabilities which sum to 1 . This vector is the $\boldsymbol{\pi}$ part of $\boldsymbol{\theta}$. Each of the K entries in $\boldsymbol{\pi}$ contains the probability of that category. In our example we have $\mathrm{K}=4$ categories for the $Y$ variable, Controller, Database, Business Logic and UI. To simplify notation we will drop the dependence on $K$ and just assume that the distribution is of the correct dimension. So in generic notation it becomes

$$
Y \sim \operatorname{Cat}(\boldsymbol{\pi})
$$

We use the Dirichlet distribution as a prior on the parameter $\boldsymbol{\pi}$ of the categorical distribution. This gives us

$$
\boldsymbol{\pi} \sim \operatorname{Dir}(K, \boldsymbol{\alpha})
$$

where $\boldsymbol{\alpha}$ is a $\mathrm{K}$-dimensional vector. A draw from a $\mathrm{K}$-dimensional Dirichlet distribution returns a K-dimensional probability distribution, which is exactly what we need for our Categorical distribution. Again, to simplify notation we will drop the $K$ and just assume that the Dirichlet is of the correct dimension. So the notation becomes

$$
\boldsymbol{\pi} \sim \operatorname{Dir}(\boldsymbol{\alpha})
$$

For the sake of brevity, inside equations 4.132 is expressed as:

$$
\operatorname{Dir}(\boldsymbol{\pi} \mid \boldsymbol{\alpha})
$$

\section{Probability Distributions on $X$}

Next, we come to the conditional probabilities for variables given the component (the $p(\boldsymbol{X} \mid Y, \theta)$ part of 4.128). As in the ordinary Naive Bayes discussed in Section 4.3 we will make the naive assumption that the variables in $\boldsymbol{X}$ are independent. With this assumption we can factor $\boldsymbol{X}$ given $Y$ and $\boldsymbol{\theta}$ as:

$$
p(\boldsymbol{X} \mid Y=k, \boldsymbol{\theta})=\prod_{p=1}^{P} p\left(X_{p} \mid Y=k, \boldsymbol{\theta}\right)
$$

Where $P$ is the number of input variables in $\boldsymbol{X}$. In our case $P=3$, Customer, Site, and Priority (remember that in this example we ignore the textual parameters Heading and Observation). For example, if we study 
the Site variable of the $i$ :th observation, $\left(x_{i, 2}\right)$, this prior would tell us $p\left(\right.$ Site $=x_{i, 2} \mid$ Component $=$ Controller,$\left.\theta\right)$, i.e. the probability that the Site component of $\boldsymbol{X}$ equals the outcome $x_{i, 2}$ given that the component is Controller. Since each of our individual input variables $\left(X_{p}\right)$ are also categorical.

$$
X_{p} \mid Y=k, \boldsymbol{\theta} \sim \operatorname{Cat}\left(\phi_{\boldsymbol{k}, \boldsymbol{p}}\right)
$$

We again use the Dirichlet distribution for the prior on the parameters of this conditional categorical distribution. Where $\phi_{\boldsymbol{k}, \boldsymbol{p}}$ is the vector of probabilities, from $\boldsymbol{\theta}$, for the outcome states for the $p$ :th variable given that $Y=k$. The full matrix for all classes and variables is $\boldsymbol{\phi}$. Since $\boldsymbol{\phi}_{\boldsymbol{k}, \boldsymbol{p}}$ is a probability distribution we again choose a Dirichlet for the prior on $\phi$ of a variable $\boldsymbol{X}_{\boldsymbol{p}}$ given a class label $k$ :

$$
\phi_{\boldsymbol{k}, \boldsymbol{p}} \sim \operatorname{Dir}\left(\boldsymbol{\beta}_{\boldsymbol{k}, \boldsymbol{p}}\right)
$$

$\boldsymbol{\beta}$ is a matrix with hyper parameters on $\boldsymbol{\phi}$. Often $\boldsymbol{\beta}$ is simply a scalar which is divided by the number of entries needed in respective vector, but conceptually and in our derivations we will use the full matrix representation. $\phi$ and $\boldsymbol{\beta}$ are of dimension $K x P x J_{p}$ where $K$ is the number of classes and $P$ the number of variables and $J_{p}$ the number of possible outcome states of variable $p$. Note that sometimes we ignore the $J_{p}$ dimension to simplify notation. So $\boldsymbol{\phi}_{\boldsymbol{k}, \boldsymbol{p}}$ is a vector with probabilities (of length $J_{p}$ ), where each entry represents the probability of an outcome for variable $p$ given that $y$ has class $k$. The prior on $\phi_{\boldsymbol{k}, \boldsymbol{p}}$ is a Dirichlet with a vector (also of length $J_{p}$ ) with hyper parameters $\boldsymbol{\beta}_{\boldsymbol{k}, \boldsymbol{p}}$. This means that we have one Dirichlet distribution as prior per variable in the model and per class $K$. I.e one for $\mathrm{p}($ Site $\mid$ Component=k) one for $\mathrm{p}($ Customer $\mid$ Component $=\mathrm{k})$ and one for $\mathrm{p}($ Priority $\mid$ Component $=\mathrm{k})$ for all $k$, each of which is a vector with probabilities (i.e. a discrete probability distribution).

With the two priors $\pi$ and $\phi$ we have introduced another two sets of parameters $\alpha$ and $\beta$, don't we need priors on these then also? Well, we could, but somewhere we have to stop, and while we stop here for this example, we could in principle keep adding prior distributions as long as we wanted (in theory at least, in practice, deep hierarchical models can cause the inference procedure to take a long time). When we have parameters in the model with no priors, these are called hyper parameters. Hyper parameters can be selected based on domain knowledge. Typically the hyper parameters are selected as to give as little information as possible to the model but still being reasonable. There is too much to be said about selecting hyper parameters and priors to cover in this thesis, we refer to Gelman et al. [36] for further discussion on the topic. 


\section{Posterior Probability}

We can now expand Equation 4.128 (repeated here for convenience) with the details on the individual probability distributions.

$$
p(Y \mid \boldsymbol{X}, \text { Data }) \propto \int p(\boldsymbol{X} \mid Y, \boldsymbol{\theta}) p(Y \mid \boldsymbol{\theta}) p(\text { Data } \mid \boldsymbol{\theta}) p(\boldsymbol{\theta}) d \boldsymbol{\theta}
$$

$p(\boldsymbol{X} \mid Y, \boldsymbol{\theta})$ (probability of a new $\boldsymbol{X}$ given $Y$ and $\boldsymbol{\theta}$ ) only depends on the $\boldsymbol{\phi}$ part of $\boldsymbol{\theta}$ so it becomes:

$$
p(\boldsymbol{X} \mid Y=c, \boldsymbol{\theta})=\prod_{k, p} \operatorname{Cat}\left(X_{p} \mid \boldsymbol{\phi}_{\boldsymbol{k}, \boldsymbol{p}}\right)^{I(k=c)}
$$

For notational simplicity we express the combined product $\prod_{k=1}^{K} \prod_{p=1}^{P}$ as $\prod_{k, p}$. The indicator function makes sure we select the correct class and reduces the other classes to ones so the product still works. For the Categorical distribution, $\operatorname{Cat}\left(\boldsymbol{X}_{\boldsymbol{p}} \mid \phi_{\boldsymbol{k}, \boldsymbol{p}}\right)$ reduces to $\phi_{k, p, X_{p}}$, i.e. the $X_{p}$ :th entry in the vector $\phi_{\boldsymbol{k}, \boldsymbol{p}}$. Remember that $\boldsymbol{\phi}_{\boldsymbol{k}, \boldsymbol{p}}$ is the vector of probabilities for the outcomes of the $p$ :th variable when the class $=k$. So for the Customer variable $(p=1)$ the $X_{3}$ :rd entry would be Customer $3 . \phi_{1,1,1}$ is the probability that the first variable (Customer) takes the 1:st outcome Customer 1 given that $Y$ has class 1 (Controller).

$$
p(\boldsymbol{X} \mid Y=c, \boldsymbol{\theta})=\prod_{k, p} \phi_{k, p, X_{p}}^{I(k=c)}
$$

Equation 4.138 expresses the probability of a new unseen vector variable given that the class of $Y=c$ and $\boldsymbol{\theta}$ (more specifically the $\boldsymbol{\phi}$ part of $\boldsymbol{\theta}$ ).

$p(Y \mid \boldsymbol{\theta})$ which is the probability of $Y$ given $\boldsymbol{\theta}$ only depend on the $\boldsymbol{\pi}$ part of $\boldsymbol{\theta}$ so it is by definition:

$$
p(Y=c \mid \boldsymbol{\theta})=\pi_{c}
$$

Where $\pi_{c}$ is the $c$ :th entry in $\boldsymbol{\pi}$.

$p(\boldsymbol{D a t a} \mid \boldsymbol{\theta}) p(\boldsymbol{\theta})$ is the product of the likelihood of all of the observations in the dataset times the prior on $\boldsymbol{\theta}$. Remember that Data $=\left(x_{1}, y_{1}\right), \ldots,\left(x_{n}, y_{n}\right)$. The first part of this expression becomes:

$$
\begin{aligned}
p(\boldsymbol{D a t a} \mid \boldsymbol{\theta}) & =\prod_{i=1}^{N}\left[p\left(y_{i} \mid \boldsymbol{\pi}\right) \prod_{k, p} p\left(x_{i, p} \mid \boldsymbol{\phi}_{\boldsymbol{k}, \boldsymbol{p}}\right)^{I\left(y_{i}=k\right)}\right] \\
& =\prod_{i=1}^{N}\left[\operatorname{Cat}\left(y_{i} \mid \boldsymbol{\pi}\right) \prod_{k, p} \operatorname{Cat}\left(x_{i, p} \mid \boldsymbol{\phi}_{\boldsymbol{k}, \boldsymbol{p}}\right)^{I\left(y_{i}=k\right)}\right]
\end{aligned}
$$

and $p(\boldsymbol{\theta})$ is our factored prior, so we can treat $\boldsymbol{\pi}$ and $\boldsymbol{\phi}$ separately 


$$
\begin{aligned}
p(\boldsymbol{\theta}) & =p(\boldsymbol{\pi}) \prod_{k, p} p\left(\boldsymbol{\phi}_{\boldsymbol{k}, \boldsymbol{p}}\right) \\
& =\operatorname{Dir}(\boldsymbol{\pi} \mid \boldsymbol{\alpha}) \prod_{k, p} \operatorname{Dir}\left(\boldsymbol{\phi}_{\boldsymbol{k}, \boldsymbol{p}}\right)
\end{aligned}
$$

combining $p($ Data $\mid \boldsymbol{\theta})$ and $p(\boldsymbol{\theta})$ yields

$p(\boldsymbol{D a t a} \mid \boldsymbol{\theta}) p(\boldsymbol{\theta})=\prod_{i=1}^{N}\left[\operatorname{Cat}\left(y_{i} \mid \boldsymbol{\pi}\right) \prod_{k, p} \operatorname{Cat}\left(x_{i, p} \mid \boldsymbol{\phi}_{\boldsymbol{k}, \boldsymbol{p}}\right)^{I\left(y_{i}=k\right)} \operatorname{Dir}(\boldsymbol{\pi} \mid \boldsymbol{\alpha}) \prod_{k, p} \operatorname{Dir}\left(\boldsymbol{\phi}_{\boldsymbol{k}, \boldsymbol{p}}\right)\right]$

Equation 4.144 is proportional to the posterior on the parameter $\boldsymbol{\theta}$ given the data. We gather the factors related to $\pi$ and $\phi$

$$
p(\boldsymbol{D a t a} \mid \boldsymbol{\theta}) p(\boldsymbol{\theta})=\prod_{i}^{N} \quad \underbrace{\left[\operatorname{Dir}(\boldsymbol{\pi} \mid \boldsymbol{\alpha}) \operatorname{Cat}\left(y_{i} \mid \boldsymbol{\pi}\right)\right]}
$$

A: probability of observation $y_{i}$ taking its observed class given $\pi$

$$
\prod_{i=1}^{N} \underbrace{\prod_{k, p}\left[\operatorname{Dir}\left(\boldsymbol{\phi}_{\boldsymbol{k}, \boldsymbol{p}} \mid \boldsymbol{\beta}_{\boldsymbol{k}, \boldsymbol{p}}\right) \operatorname{Cat}\left(x_{i, p} \mid \boldsymbol{\phi}_{\boldsymbol{k}, \boldsymbol{p}}\right)^{I\left(y_{i}=k\right)}\right]}_{\begin{array}{l}
\text { B: probability of variable } X_{i} \text { 's } p: \text { th } \\
\text { entry taking value } x_{i, p} \text { given } \boldsymbol{\phi} \text { and } y_{i}=k
\end{array}}
$$

The first factor in 4.145 (denoted A) simplifies (due to the DirichletCategorical conjugacy described in 4.4) to:

$$
\operatorname{Dir}\left(\boldsymbol{\pi} \mid \boldsymbol{\alpha}+\boldsymbol{C}_{\boldsymbol{c}}\right)
$$

$\boldsymbol{C}_{\boldsymbol{c}}$ is a K-dimensional vector of counts of the number of times in the whole dataset the outcome $y$ belongs to the class $c$. The second factor in 4.145 (denoted B) simplifies, also due to the Dirichlet-Categorical conjugacy, to:

$$
\operatorname{Dir}\left(\phi_{\boldsymbol{k}, \boldsymbol{p}} \mid \boldsymbol{\beta}_{\boldsymbol{k}, \boldsymbol{p}}+\boldsymbol{C}_{\boldsymbol{c}, \boldsymbol{p}}\right)
$$

$\boldsymbol{C}_{\boldsymbol{c}, \boldsymbol{p}}$ is the vector with counts of the outcomes of variable $p$ when $Y$ has class $c$. The indicator goes away since it has done its job and selected out only the counts for class $c$. As an example, if we look at the Customer variable, the counts for the different outcomes when $Y=$ Controller and Controller is encoded as index 1 may be

$$
C_{k=1, p=1}=(\text { Customer } 1=5, \text { Customer } 2=7, \ldots, \text { Customer } 12=3)
$$

Denoting that when $Y=$ Controller (encoded as $k=1$ ) the Customer variable (encoded as $p=1$ ) had the outcome Customer 1 five times, Customer 2 seven 
times, etc. The $\boldsymbol{C}_{\boldsymbol{c}, \boldsymbol{p}}$ counts is then added to the prior counts in $\boldsymbol{\beta}_{\mathbf{1 , 1}}$ to yield the posterior parameters for the Dirichlet in Equation 4.148. Since we have now gathered the counts, the product over $\mathrm{N}$ disappears and our new expression becomes:

$$
p(\boldsymbol{D a t a} \mid \boldsymbol{\theta}) p(\boldsymbol{\theta})=\operatorname{Dir}\left(\boldsymbol{\pi} \mid \boldsymbol{\alpha}+\boldsymbol{C}_{\boldsymbol{c}}\right) \prod_{k, p} \operatorname{Dir}\left(\boldsymbol{\phi}_{\boldsymbol{k}, \boldsymbol{p}} \mid \boldsymbol{\beta}_{\boldsymbol{c}, \boldsymbol{p}}+\boldsymbol{C}_{\boldsymbol{c}, \boldsymbol{p}}\right)
$$

We can now start to put together our final expression for the posterior predictive distribution for a new $Y$ taking the class $c$

$$
p(Y=c \mid \boldsymbol{X}, \boldsymbol{D a t a}) \propto \int \underbrace{\pi_{c} \prod_{k, p} \phi_{k, p}^{I(k=c)}}_{p(Y \mid \boldsymbol{\theta}) p(\boldsymbol{X} \mid Y=c, \boldsymbol{\theta})} \underbrace{\operatorname{Dir}\left(\boldsymbol{\pi} \mid \boldsymbol{\alpha}+\boldsymbol{C}_{\boldsymbol{c}}\right) \prod_{k, p} \operatorname{Dir}\left(\boldsymbol{\phi}_{\boldsymbol{k}, \boldsymbol{p}} \mid \boldsymbol{\beta}_{\boldsymbol{k}, \boldsymbol{p}}+\boldsymbol{C}_{\boldsymbol{k}, \boldsymbol{p}}\right) d \boldsymbol{\theta}}_{p(\boldsymbol{\theta}) p(\text { Data } \mid \boldsymbol{\theta})}
$$

We again collect the variables that are related

$$
p(Y=c \mid \boldsymbol{X}, \text { Data }) \propto \int \pi_{c} \operatorname{Dir}\left(\boldsymbol{\pi} \mid \boldsymbol{\alpha}+\boldsymbol{C}_{\boldsymbol{c}}\right) \prod_{k, p} \boldsymbol{\phi}_{\boldsymbol{k}, \boldsymbol{p}}{ }^{I(k=c)} \prod_{k, p} \operatorname{Dir}\left(\boldsymbol{\phi}_{\boldsymbol{k}, \boldsymbol{p}} \mid \boldsymbol{\beta}_{\boldsymbol{k}, \boldsymbol{p}}+\boldsymbol{C}_{\boldsymbol{k}, \boldsymbol{p}}\right) d \boldsymbol{\theta}
$$

Move in related factors under common product and split the integral into two parts

$$
\begin{aligned}
p(Y=c \mid \boldsymbol{X}, \text { Data }) & \propto \int \pi_{c} \operatorname{Dir}\left(\boldsymbol{\pi} \mid \boldsymbol{\alpha}+\boldsymbol{C}_{\boldsymbol{c}}\right) d \boldsymbol{\pi} \\
& \int \prod_{k, p} \boldsymbol{\phi}_{\boldsymbol{k}, \boldsymbol{p}}{ }^{I(k=c)} \operatorname{Dir}\left(\boldsymbol{\phi}_{\boldsymbol{k}, \boldsymbol{p}} \mid \boldsymbol{\beta}_{\boldsymbol{k}, \boldsymbol{p}}+\boldsymbol{C}_{\boldsymbol{k}, \boldsymbol{p}}\right) d \boldsymbol{\phi}
\end{aligned}
$$

Where in the last part, the integral and product can be interchanged to yield

$$
\prod_{k, p} \int \phi_{\boldsymbol{k}, \boldsymbol{p}}{ }^{I(k=c)} \operatorname{Dir}\left(\boldsymbol{\phi}_{\boldsymbol{k}, \boldsymbol{p}} \mid \boldsymbol{\beta}_{\boldsymbol{k}, \boldsymbol{p}}+\boldsymbol{C}_{\boldsymbol{k}, \boldsymbol{p}}\right) d \boldsymbol{\phi}
$$

We can move the integral inside the product since for a fixed $j$ and $k$ the others are constant. If we study Equation 4.153 we can conclude that this is the product of a set of expected values. Since the expected value of the $\mathrm{RV} R$ is $E[R]=\int_{-\infty}^{\infty} r * p(r) d r$ we see that the first part of Equation 4.151 is the expected value of $\boldsymbol{\pi}$ w.r.t $\operatorname{Dir}\left(\boldsymbol{\pi} \mid \boldsymbol{\alpha}+\boldsymbol{C}_{\boldsymbol{c}}\right)$ and the second part are the products of the expected values of $\boldsymbol{\phi}$ w.r.t. $\operatorname{Dir}\left(\boldsymbol{\phi}_{\boldsymbol{k}, \boldsymbol{p}} \mid \boldsymbol{\beta}_{\boldsymbol{k}, \boldsymbol{p}}+\boldsymbol{C}_{\boldsymbol{k}, \boldsymbol{p}}\right)$. Since we know the expected value of a Dirichlet distributed variable we can write down a simplified expression for the probability of $Y=c$

$$
p(Y=c \mid \boldsymbol{X}, \boldsymbol{D a t a}) \propto E[\boldsymbol{\pi}] \prod_{k, p} E\left[\boldsymbol{\phi}_{\boldsymbol{k}, \boldsymbol{p}}\right]
$$


We can look up what the expected value of a Dirichlet is. The general expression for the expected value of a Dirichlet is:

$$
E\left[\pi_{c}\right]=\frac{\alpha_{c}}{\sum_{c} \alpha_{c}}
$$

Which in our case for $\pi$ becomes

$$
\int \pi_{c} \operatorname{Dir}\left(\boldsymbol{\pi} \mid \boldsymbol{\alpha}+\boldsymbol{C}_{\boldsymbol{c}}\right) d \boldsymbol{\pi}=E\left[\pi_{c}\right]=\frac{\alpha_{c}+c_{c}}{\alpha_{0}+N}
$$

where $\alpha_{0}$ is the sum of all $\alpha_{c}$ and $N$ is the sum of all $C_{c}$ (vector of counts for each class), which is just the number of observations. $c_{c}$ (observe non-bold small letter) is the counts for class $c$. Correspondingly, the expression for $\phi$ then becomes

$$
\prod_{k, p} \int \boldsymbol{\phi}_{\boldsymbol{k}, \boldsymbol{p}}^{I(k=c)} \operatorname{Dir}\left(\boldsymbol{\phi}_{\boldsymbol{k}, \boldsymbol{p}} \mid \boldsymbol{\beta}_{\boldsymbol{k}, \boldsymbol{p}}+\boldsymbol{C}_{\boldsymbol{k}, \boldsymbol{p}}\right) d \boldsymbol{\phi}=\prod_{k, p} E\left[\boldsymbol{\phi}_{\boldsymbol{c}}\right]=\prod_{p} \frac{\beta_{c, x_{p}}+C^{c, x_{p}}}{\beta_{0, p}+C_{c}}
$$

The $C_{k, p}$ disappears and is replaced with $C^{c, x_{p}}$ since the indicator function selects out only the counts where $k=c$. For each factor $k \neq c$ in the product, the other factors are the integral over a full probability distribution which reduces to 1 . $C^{c, x_{p}}$ is the count of the state $x_{p}$ when $Y$ has class $c$. This gives us our final expression for the probability of the class $Y$ of a new data point $\boldsymbol{X}$ given the historical data

$$
p(Y=c \mid \boldsymbol{X}, \boldsymbol{D a t a}) \propto \frac{\alpha_{c}+c_{c}}{\alpha_{0}+N} \prod_{p} \frac{\beta_{c, x_{p}}+C^{c, x_{p}}}{\beta_{0, p}+C_{c}}
$$

Equation 4.154 gives an expression that is proportional to the probability, and for classification this is all we need. The first part is the prior counts for the class plus the observed counts for the class divided by the sum of all prior counts plus the total number of observations. The second part is the product over all the input variables. The $\beta_{c, p}$ is the prior counts for variable $p$ for class $c$. The $C_{c, p}$ are the observed counts of the $p$ 'th variable, and the denominator is the corresponding sums. We can now evaluate Equation 4.154 for all the classes and select the class which has the highest value, i.e. $Y$ is assigned the class $c$ that maximizes Equation 4.154.

$$
Y \leftarrow c=\underset{c}{\arg \max } p(Y=c \mid \boldsymbol{X}, \boldsymbol{D a t a}) \propto \frac{\alpha_{c}+c_{c}}{\alpha_{0}+N} \prod_{p} \frac{\beta_{c, x_{p}}+C^{c, x_{p}}}{\beta_{0, p}+c_{c}}
$$

Equation 4.155 gives us a Bayesian Naive Bayes classifier for categorical data, where we get the smoothing, "for free", as part of the model definition. If we compare with Equation 4.56 in Section 4.3 (repeated here for convenience) for the simple Naive Bayes with smoothing 


$$
Y \leftarrow c=\underset{c}{\arg \max } p(Y=c \mid \boldsymbol{X}, \boldsymbol{D a t a}) \propto \frac{1+c_{c}}{k+N} \prod_{p} \frac{1+C^{c, x_{p}}}{C_{p, s}+c_{c}}
$$

we see that the 1 in the nominator in the first factor is replaced with the prior $\alpha_{c}$ and the $k$ with the corresponding $\alpha_{0}$. Likewise under the product the 1 in the nominator is replaced with the prior $\beta_{c, x_{p}}$ and its corresponding $\beta_{0, p}$ in the denominator. If we set these priors to 1 we get back the smoothed simple Naive Bayes model.

In this discrete case we could normalize by summing over all the classes and divide the proportional probability for each of the classes with this sum. We could further derive equations for the uncertainty in the classification and parameter estimates, but that is beyond the scope of this chapter. Paper IV describes a more advanced Bayesian classifier and Paper V shows the application of that classifier to the bug localization problem.

\subsection{Visualization}

Visualization is an important part of data science, both in communicating results and in the data exploration steps. Oftentimes a researcher wants to explore a dataset to try to find some meaningful "patterns" without knowing beforehand what to look for. Traditional methods for visualization include histograms, time series plots, scatter plots and pie charts. These traditional low dimensional methods can be hard to apply on high dimensional datasets. Recent advances in dimensionality reduction techniques by Maaten and Hinton [38] has enabled exploration of high dimensional datasets using a technique called t-Distributed Stochastic Neighbor Embedding (t-SNE).

Say for instance that we would like to visualize our bug database. How would we do this? We could present stats in pie chart or histogram form which customers reports the most bugs or the inflow of high priority bugs per week. But if we where interested in visualizing the bug reports based on the contents of the descriptions of the bug reports, then what should we do? One option is to represent the bug report text using LDA topics. I.e. we take the text descriptions of the bug reports and run them through LDA. We then get the topic distribution for each of the bug reports as a vector of probabilities, one for each LDA topic. We now have a high dimensional dataset, but with a fixed dimension, $\mathrm{N}$, the number of bug reports and $\mathrm{K}$, the number of LDA topics.

We can now take the resulting NxK matrix, and run it through t-SNE to get a 2D-representation of that dataset. Figure 4.9 shows the resulting graph of such an exercise. In the graph, we clearly see clusters of bug reports. We can now go in and study bug reports clusters and try to understand what makes these bug reports similar. Since they are close in the t-SNE generated graph, they have similar topic structures. 


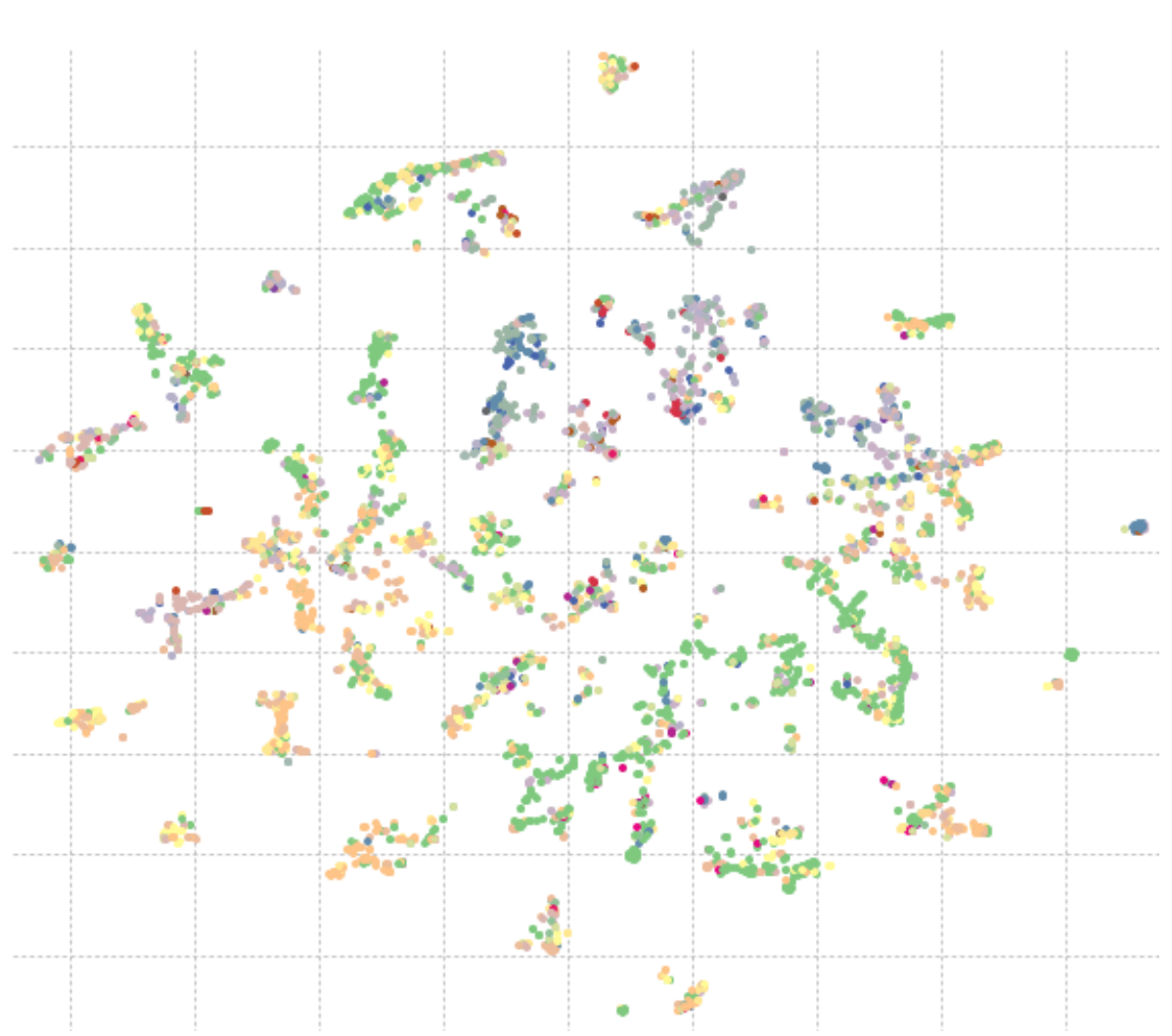

Figure 4.9: t-SNE rendering of a bug database using the unstructured text encoded as LDA topic distributions as inputs. The color represents the class of the bug report.

This is one alternate way to explore bug reports on an organizational level to try to understand themes or topics in an organizations bug repository. As part of the exploratory work in this thesis we have implemented a Java and Julia framework for working with t-SNE and released it Open Source. Aside from providing a Java implementation we also show (as part of the implementation) that the Barnes-Hut approximation (Van Der Maaten [39]) can be efficiently parallelized. 



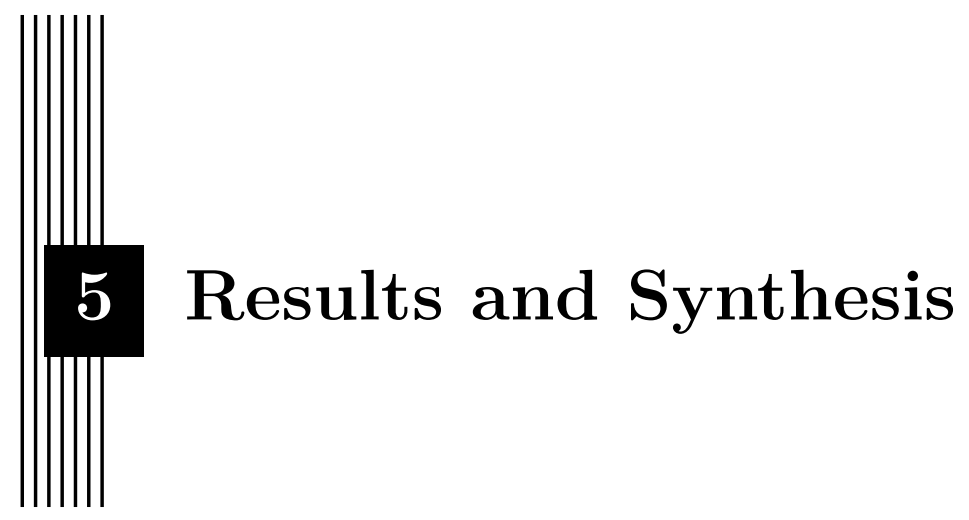

In this section we discuss our research questions by synthesizing the material from the included papers.

For the reader's convenience, we repeat our originally stated research questions from Section 1.4.

1. RQ1: How well, in terms of accuracy, can we expect machine learning techniques to perform? Is it feasible to replace human bug assignment and fault localization with machine learning techniques in large scale industry SW development projects?

2. RQ2: Which machine learning techniques should be used in large-scale industry settings?

3. RQ3: How can we improve ML techniques, aside from increasing prediction accuracy, to increase their usefulness in AFL and ABA?

In answering RQ1 we look at a couple of different measures. The evaluation metric we have focused mostly on in our papers is the so called cross-fold validation $(\mathrm{CV})$ approach, more specifically $\mathrm{k}$-fold cross-validation. We have preferred mainly 10-fold CV over Leave-One-Out CV, both for computational and theoretical [40] reasons. The reason for focusing on cross validation, even though it has some problems [28], is that it is a widely used, and wellrecognized technique for estimating the generalization error of classifiers. It also has the advantage that it is quite intuitive to understand for stakeholders 
with limited statistical background. In Paper II we complement the use of $\mathrm{CV}$ with other types of evaluation approaches, which we believe shows some of the limitations of $\mathrm{CV}$ in estimating the generalization error.

Looking at results from our cross validation studies we are getting average prediction accuracies in the range of between $50 \%$, to over $90 \%$. Whether it is feasible to replace human bug assignment and fault localization will be very project specific. We see from our studies on real systems in Paper II, that the prediction accuracy vary substantially from project to project. We have reasoned that if an ML-system can reach a prediction accuracy level close of that of humans, but can perform the predictions in practically no time, we have considered it feasible to replace humans. Then again, some organization might reason that if the bug assignment takes in practice no-time to perform, then they could accept a lower prediction accuracy than that of humans. Another aspect to take into consideration is how much variance in prediction accuracy an organization is willing to accept. We have looked at average prediction accuracy, but perhaps for social reasons, an ML-system won't be accepted unless it has a very low variance in accuracy. Although we have not reported it, the variance in our cross validations have been quite low. This is likely due to the quite large amount of data that we have used. Some further aspects for consideration; perhaps an organization is willing to use an MLsystem for low priority bugs but use human routing for high priority bugs. In Paper V we explored a richer ML approach than in the earlier papers. There we conclude that if the ML-system can supply a measure of uncertainty, an organization can use this to trade automation level versus prediction accuracy. This is achieved by only letting the ML-system act on bug reports of which it is very confident.

In Paper I we introduce the Stacked Generalizer and evaluate it on one large-scale telecommunications system. We show that the approach achieves a cross-fold prediction accuracy close to that of humans (ML $76 \%$, humans 77 $\%$ ) on the same system when evaluated on what we call external bug reports. External bug reports are, in this context, bug reports that have not been written by the design teams themselves. Using only external bug reports is an important methodological consideration, since otherwise we risk getting an unfairly poor comparison with human classifiers. This is because humans who already know that a bug report has been written on a specific team obviously have a distinct advantage in routing those bug reports. Furthermore, it does not make sense to use the suggested approach on bug reports that the team have written on their own components because then the organization already knows which team should handle the bug report and no routing is needed. The paper also shows that the prediction accuracy is consistently better when using the Stacked Generalizer than when using only one individual classifier. Paper II shows similar results as Paper I, and even though alternative evaluation methods to $\mathrm{CV}$ indicates lower prediction accuracies than $\mathrm{CV}$, considering our lack of optimization and feature engineering, we still believe ML can have 
reached the utility breakpoint we discuss in Paper II. In Paper V we get quite high prediction accuracy if we require the ML-system to be very certain of its predictions. These results makes us cautiously answer the second part of RQ1 affirmatively, with the understanding that more work needs to be done before a complete "swap out" is feasible.

In turning to the first part of RQ1, Paper II further explores the use of Stacked Generalizers by a deeper analysis of its performance compared to individual classifiers, on more systems, with more varied analysis methods. Paper II is, as far as we know, still the largest evaluation available of automated bug triaging on industry data. The approach is evaluated on four different telecommunications systems and one large system for industrial automation, making up a dataset of more than 50,000 bug reports.

Again in Paper II, the Stacked Generalizer performs better than the individual classifiers, although in some cases just marginally. The overall conclusion is that in some cases the Stacked Generalizer is either no better or just marginally better than any one single classification algorithm, but in no case is it worse. And even though it is sometimes just as good as the best algorithm, the challenge for the organization is then to find which algorithm is best for the particular combination of data and parameters. Our results in Paper II also show that when it comes to ensemble selection, the naive approach of selecting the best of the individual classifiers, works as well as an approach where we try to find complimentary classifiers by thinking about the learning strategy of the individual classifiers. We need to be a bit cautious when interpreting this particular result, since the methodology of ensemble selection led to quite similar ensembles in both cases, so it is unclear if this result generalizes.

In Paper II we also investigate the effects of including unstructured text as predictors. The results show that including both text and structured variables improves performance over having only text or structured variables. For all five cases studied in Paper II, the prediction accuracy started to flatten out when reaching around 2000 bug reports. This led us to conclude that if a bug triaging problem has somewhere between 17 - 65 teams, at least around 2000 bug reports should be available before an organization should start evaluating ML-based bug triaging.

In Paper II, results show that in four out of five systems, prediction accuracy declines with the age of the bug reports. I.e., as training data gets older, it get worse at predicting current bugs. The rate of the decrease was not constant over all systems. Some had a very fast decline in prediction accuracy, with the accuracy halving in roughly 100 days, while others showed a much slower decline. This would almost certainly be dependent on the structure of the software being developed. With rapidly changing software, we can reason that the decline would be faster since older software is very different from new software that is being developed. 
Early in the development of Papers I and II came the question of feature selection, particularly how to handle unstructured text as input to machine learning algorithms. In Paper II this question was highlighted with a small study, where we tested different combinations of using no text, text only and combination of text and other covariates. In all cases the combination of both text and other covariates gave the best results. In Paper II we speculated that more sophisticated handling of the unstructured text input could possibly lead to better classification accuracy. In both Papers I and II, the simple TF-IDF approach was used to model text. The search for a better approach to modeling text led to the study of LDA. The reasons for selecting LDA were as follows:

- Robustness on non-well behaved data

- Unlike, for instance, Natural Language Processing (NLP), LDA is robust to text data that is not syntactically correct according to either formal grammars or even informal grammars such as, for example, the English language. This is because LDA does not try to model the syntactic structure or grammar of the text, but only models the clustering behaviour of individual words in the text

- In our data we frequently have a mix of human and machine generated text

- Fast

- LDA is faster on large copora than, for instance, NLP approaches

- Interpretable

- LDA is interpretable on a modeling level since it is a well-defined Hierarchical Bayesian model

- LDA is interpretable on a semantic level since it defines themes in text that are understandable by humans

We discovered that all of the prevailing fast algorithms for inference in the LDA model were approximations to the LDA model based on the AD-LDA approach by Newman, Asuncion, Smyth, and Welling [41]. In Paper III we develop a fast and mathematically correct (i.e. it is guaranteed to converge to the correct posterior LDA distribution) Gibbs Sampler for the LDA model. In this paper we show that on shared memory architectures, our correct sampler for LDA is competitive with all approximate AD-LDA based models and the state-of-the-art Light-LDA by Yuan, Gao, Ho, Dai, Wei, Zheng, Xing, Liu, and Ma [42]. The Light-LDA sampler also uses the same approximation as AD-LDA, but with a slightly different sampling scheme. We show some of the advantages of the exact model by simultaneously developing a so-called Spikeand-Slab variable selection model for LDA. This type of variable selection is 
only possible with an exact sampler that retains the so-called $\phi$ matrix in LDA.

Another reason for developing an exact sampler for LDA was our suspicion that approximate LDA models may be fine when humans are interpreting the output topics of LDA. But we feared that when basing a classifier on the output of an approximate LDA, the incurred approximation could result in worse prediction accuracy. This is something we would not need to worry about with an exact sampler. We have not verified our suspicion, but we have the benefit of not having to worry about approximation errors (other than MCMC errors). Our work with LDA partly answers RQ3; with non-approximate implementations we don't have to worry about error-propagation, especially one that potentially increases with parallelization.

After having developed a fast, correct, and parallel sampler for LDA, we continued on our path to a classifier that could combine structured data in the form of nominal and numerical data, and unstructured text with a bit more sophistication than simple TF-IDF. Also, by this time we had realized, based on feedback on the Stacked Generalizer approach, that the interpretability of the output of the classifier was also very important. This observation is also reported in research by Parnin and Orso [26]. This is a weakness of the Stacked Generalizer, the output of which is hard to interpret. To this end, in Paper IV we develop a Hierarchical Bayesian Classifier based on a combination of LDA and the so-called Diagonal Orthant Probit classification model. As a mnemonic for Diagonal Orthant (DO) in combination with LDA we named the classifier DOLDA ${ }^{1}$.

The experiences from Papers I and II, give a nuanced answer to RQ2. If prediction accuracy is important and there is limited ML knowledge in an organization, Stacked Generalization is a good alternative. Stacking is robust and provides good prediction accuracy without the need for large amounts of expertise in ML, and it allows combinations of many different types of classifiers. It is possible that other techniques such as Deep Learning (DL) [43] can give even better prediction accuracy, but DL techniques typically require expert knowledge and a large hardware investment to deploy due to their high computational requirements. If interpretability is a requirement then simpler models that are easier to understand are required. If an organization wants to trade an amount of prediction accuracy for model flexibility and interpretability, other approaches are necessary. For this case, our answer to RQ2 would be to employ a Bayesian model similar to our proposed DOLDA model. It gives competitive prediction accuracy and the model output is highly interpretable. It also gives a quantified uncertainty measure of all parameters in the model, including the model predictions. This gives an organization the ability to tune the desired prediction accuracy versus level of automation, as explained in Paper V. Running and using the DOLDA model

\footnotetext{
1 "dolda" is Swedish for "hidden"
} 
off-the-shelf does not require a high degree of ML expertise, but extending the model would.

In Paper IV, we develop an efficient Gibbs sampler for the DOLDA classification model. We show that the combination of Diagonal Orthant and LDA gives a competitive classification model. In the paper we study two Bayesian priors. We show that using the so-called Horseshoe prior by Carvalho et.al. [44] gives a much sparser model than using the standard normal prior. This is important because it leads to a model that is substantially easier to interpret by humans than when using a normal prior.

In Paper $\mathrm{V}$ we show that the DOLDA classification model produces competitive results on a bug classification task compared to several other models based on LDA, and also to the Stacked Generalizer approach. In addition to good classification accuracy, the DOLDA model also gives interpretable results and a mathematically correct quantification of the uncertainty of the model. We have not thoroughly investigated whether or not the correctness of our LDA sampler (rather than using the approximate AD-LDA approach to parallelism) affects the classification accuracy, but we know that we don't have to worry about model approximation error.

Paper V gives a partial answer to RQ3. Why partial? We cannot claim to have investigated all possible improvements that could be made to classifiers in this context. For example, we have not investigated any neural network or deep learning-based approaches. What is clear is that for an ML model to be maximally useful in an industrial setting it is not enough to provide a high level of prediction accuracy, it should also allow for flexibility in deployment. For instance, an organization should be able to integrate ML techniques with current manual approaches and tune the process of determining when an automated response should be enacted, and when to leave the decision to a human expert. We achieve this with the DOLDA model by ensuring that our predictions also come with a quantified uncertainty. The results from the model should be understandable by the human experts that will be involved in the bug handling process, at least as long as the automated process is being evaluated in the organization. The DOLDA model does this by giving humanunderstandable output measures in terms of weights on each of the variables in the model connected to each output class. Additionally, unstructured text is represented by topics that are understandable by humans, and the topics are given weights explaining how much each one affects each class in the classification problem. The trade-off between accuracy and interpretability will be organization specific, but we have not investigated which factors affect this trade-off.

In short, the response to RQ3 is that we can increase the usefulness of AFL and ABA techniques by ensuring that the ML models can supply a measure of uncertainty to their output and provide a rich, interpretable output model rather than just a point prediction. 


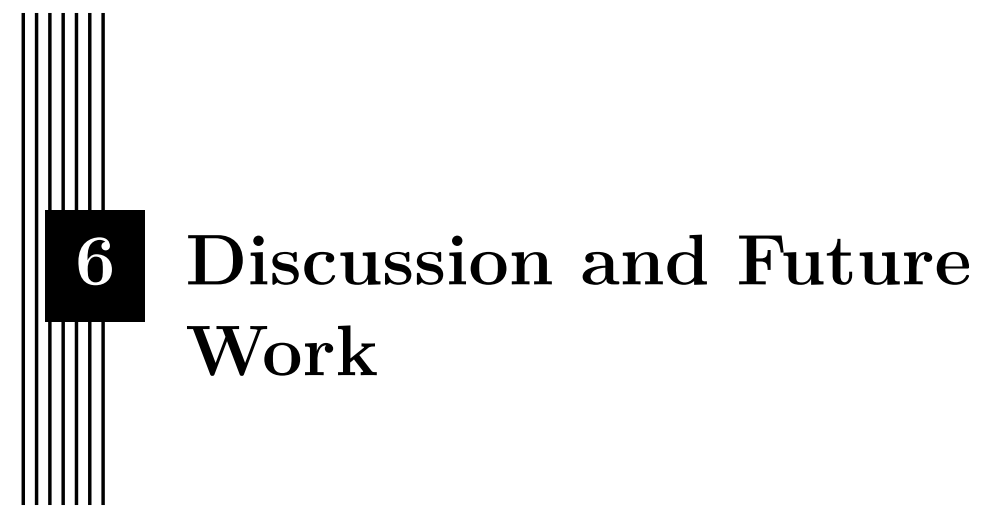

\subsection{Aspects of Industrial Adoption}

We have shown in our empirical papers (I, II, and V) that there are many possible benefits of using machine learning in the ABA and AFL contexts. Depending on the efficiency and effectiveness of the manual assignment process, an automated approach that could perform the bug assignment in a fraction of the time, around the clock, without delay, could substantially increase the efficiency in the bug assignment process.

We have focused on the technical aspects of bug assignment, but of course, deploying such a system involves many practical aspects that are not necessarily directly related to machine learning. Ways-of-working will need to be updated, new tasks related to handling errors in the ML predictions need to be dealt with, and so on. Additionally, we recommend, and believe, that most organizations would not blindly replace their current manual processes without doing some kind of pilot study. In the initial phases, tuning of the system will be essential. This should be done iteratively and in parallel, with the ML-system running side-by-side with the manual assignment. In our studies we have not optimized the classifiers or datasets in any way. This is, of course, something that should be done in the individual organizations.

We have argued that with the stacking approach, the ML skills needed by the organization would be limited. Optimizing classifiers sounds like an expert task, but much can be done by non-ML experts just by looking at the documentation of the selected frameworks. Fortunately, nowadays many of 
the large open source ML frameworks are very well documented. Another aspect of optimizing performance is minimizing the number of classes the ML system needs to consider. This can be done by merging lower level classes which can in some way be considered to belong to the same higher level class. For instance, if we have the two classes, "networking parameters" and "networking stack", these could be combined into the higher level "networking" class. This could substantially reduce errors in prediction since these similar classes tend to be mistaken for each other by the ML system. This type of optimization only requires organizational domain expertise and no particular machine learning expertise.

In Paper II, we look at how the Stacking behaves in five different projects with different numbers of bug reports. From this it is clear that the performance of the system is dependent on the unique environment. Different projects will have different learning curves. From this study, we suggest as a rule of thumb that an organization should have at least 2000 bug reports before introducing an ML-based approach. In line with other research [45], we see that older data tends to decrease prediction accuracy. This must be investigated per project and should be continuously monitored. The ML system needs to be continuously re-training as new bug reports come in and old bug reports become unrepresentative of what is happening in the system. Of course, a live system must also take project changes into consideration. For instance, changes in team setups, changes in ways of working, etc. On these occasions, the system may need re-training. Perhaps an organization wants to use a partial approach to ABA. Perhaps high priority bugs are always handled by humans while lower priority bugs are handled by the ML system.

To facilitate analysis of the system behavior, we suggest that the full bug history should be saved in a BTS. Also, the required accuracy before an organization recognizes the value of an ML system will differ between projects. In one project a prediction accuracy of $60 \%$ might be good enough, while in another $80 \%$ may be necessary. Since the automated bug assignment process is magnitudes faster, we would venture to guess that human level accuracy might not be needed before an organization found it good enough. If no ML expertise is available, we suggest starting with a Stacked Generalizer approach since it is flexible and robust. The conclusion is that even though in some cases the improvement over individual classifiers is marginal, we have never seen a case where the Stacked Generalizer (on average) performs worse than any of the individual classifiers.

Another practical aspect that we highlight in Paper II is the selection of which bug reports should be included in the training set. We observe that bug reports that are too old can actually decrease the prediction accuracy of the system. Thus, an organization needs to study which time span of bug reports best represents the current system and gives the best prediction accuracy. This might not be as simple as finding one time frame for the whole training set! Different classes might need different time spans. For instance, one class 
might have very few bug reports, so if the time frame is selected too tightly, perhaps this class will have too few samples for sufficient training. With the same time frame, another class may have too many old samples. In general, one should strive for an even distribution of observations, since training on skewed training sets can lead to bad prediction results [46, 47, 48].

Anvik [49] lists examples of aspects to consider in deployment of what he calls recommenders for bug triaging. He lists six questions which should be answered when considering designing a recommender system:

- Which reports from the BTS should be used to create the recommender?

- How many reports should be used?

- How should the reports be labeled?

- What are the valid labels?

- Which features from the reports should be used?

- Which machine learning algorithm should be used?

We conclude that all of these are also relevant in our context.

In Paper V we look at Automatic Fault Localization on the component level. We conclude that AFL can potentially be a tool for large-scale organizations to improve efficiency in the bug handling process. We show that an organization can obtain concrete benefits from already existing but often underutilized data that is available in the organization. With this approach, organizations can put data to use that is often just lying around taking up space in databases. We give examples of how an organization can use a richer classifier than the Stacked Generalizer. For instance, if the classifier supplies an uncertainty measure for its predictions, the organization can set a threshold, and if the uncertainty in the prediction exceeds the threshold, the decision can be left to a human, otherwise the ML system can perform the necessary actions. This way an organization can trade prediction accuracy for level of automation.

In conclusion, many practical aspects will determine the degree to which an ML-based approach will be beneficial to an organization, if at all. Based on our studies, we firmly believe that ML-based bug assignment and fault localization belong to the future of the software development process.

\subsection{Recommendations for an Updated Software Development Process}

What seems clear from a practical standpoint is that current software development practice needs to be updated to cater to ML aspects. A development project should analyze which aspects could facilitate the use of ML 
approaches. For instance, in this thesis we have studied ABA and AFL. For these to be automated using ML, the BTS needs to retain information on which team solved a particular bug report and in which component it was solved. Unless this information is available, it is not possible to train an ML system to automate these aspects.

It is true for all types of ML systems that better training data yields better output results. So the better data the software development process can provide, the more benefit it will get from an ML automated approach. For instance, is it possible for a machine to track the full process of a software release from a version number? Can we immediately locate which files were involved in creating the version? Which test cases have run and what are their results? Which configurations were used to test the release? Where are the $\log$ files and performance data related to a particular bug report? And so on. All of this information needs to be machine accessible both at training and prediction time.

The default view of any software project should be that its data will be analyzed by machines, not humans. Log files, for instance, should be designed for machine analysis rather than human analysis. This is not typically the case today, where developers write log outputs for other developers to read. In fact, any output from the system should be designed for machines to analyze. Not only will this benefit the machine analysis but most probably also human analysis, since the data will be amenable for processing in tools such as R and Excel, etc.

If software development projects are executed with machine analysis in mind, much of the "backpatching" can be avoided, which is otherwise needed to adapt and work around systems that are not designed to output data for machine analysis.

\subsection{Boundary Between ABA and AFL}

We have studied ABA and AFL as separate processes, but there is nothing that says that both cannot be run in parallel. An ABA system could automatically also run an AFL system. When one team only works on one subsystem AFA and ABA are equivalent, but in many cases they can be completely separate. In these cases the output of the ABA system could actually be an input to the AFL system. If we know which team should solve a bug report, that can be a strong indicator of where in the software architecture a bug is located. Combining and integrating the bug assignment with the fault localization process should be studied in future research. 


\subsection{Hierarchical Classification}

One aspect that has emerged in the process of developing DOLDA is an even richer model than DOLDA. DOLDA is a classic classifier with a flat class structure, but in many software projects the architecture is hierarchical. I.e., there are high-level systems, which consist of nodes, which in turn consist of subsystems, which are built up by components, which in turn are based on blocks, which are a set of classes. Classes in turn can consist of files, with methods, lines, tokens and finally individual characters. The ideal situation would be to have a full hierarchical classifier that would give a probability distribution on all of these levels. One example of such a hierarchy is visualized in Figure 6.1

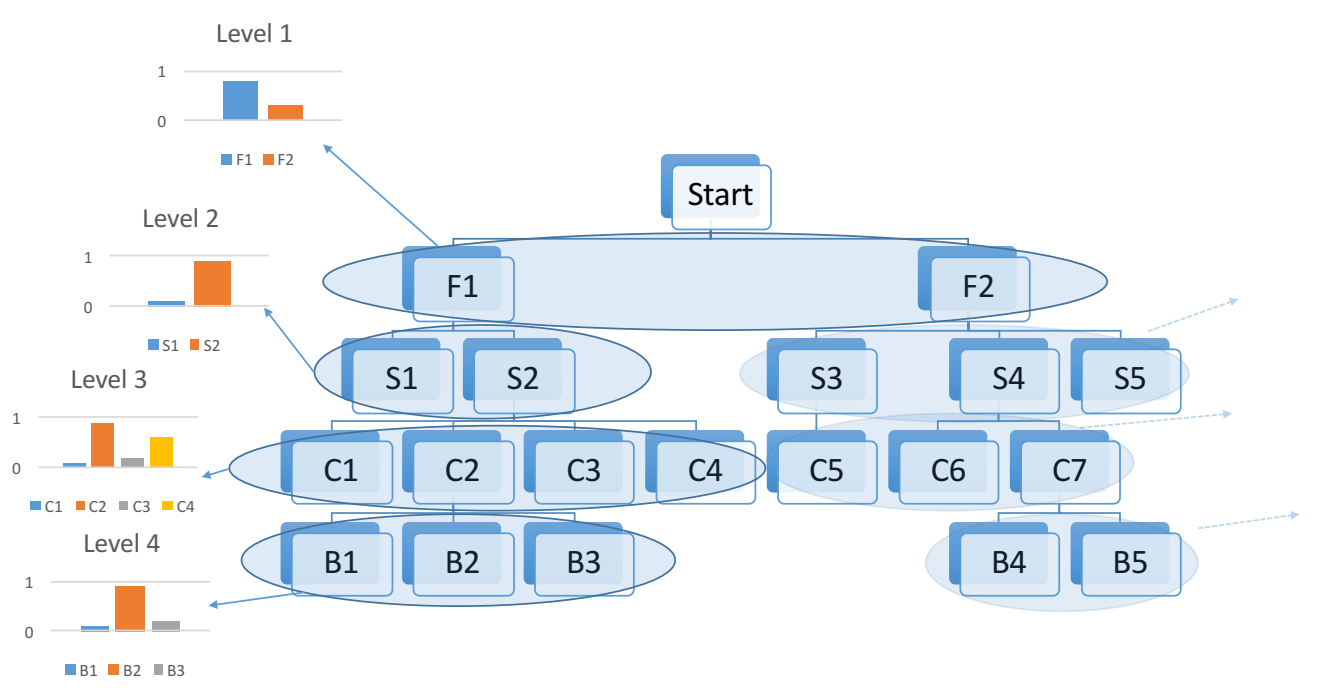

Figure 6.1: Hierarchical Classification. The F-level could be function level, S - subsystem level, C - component level and, B - block level. Histograms indicate probability per level in the tree.

This type of classification tree would provide an even richer model than a flat class structure. Extending the flat classification into hierarchical classification $[50,51]$, could be a possible future extension of our work. With this type structure, one can model the whole software architecture (or team structure), and do partial routing down the tree. The uncertainty could be used as a stopping condition, and the routing could be halted when the uncertainty gets too high. However, this puts much stricter demands on the BTS, since it needs to retain, and maintain consistency, for all of the information related to each of the levels, for every bug report. 


\subsection{Other Contextual Information}

In the software development process and during the lifetime of a deployed product, several artifacts are produced. Many of these can be used to help predict the component in which a bug is located. Exactly which information sources are available will be different for each specific product and organization, but some will undoubtedly be similar between products and even industries. Some examples of information sources are listed in Figure 6.2. Some, or perhaps all of these might be integrated into a fault localization model that can help in pinpointing where a bug is located.

Most research so far, ours included, has focused on the bug data stored in BTS'es, but there are also other sources that could be indicative of where a fault is located. For instance, if a bug is reported on a specific release of a product and there is alarm data available from a system with that release and a specific component reports an unusual number of alarms, that could be an indication that something is wrong with that particular component. Another example of additional information from Figure 6.2, is traffic data, this is often highly interesting in the Telecommunications domain. If we see a drop in the throughput of some specific type of traffic, that may be an indication that some component in that subsystem is faulty.

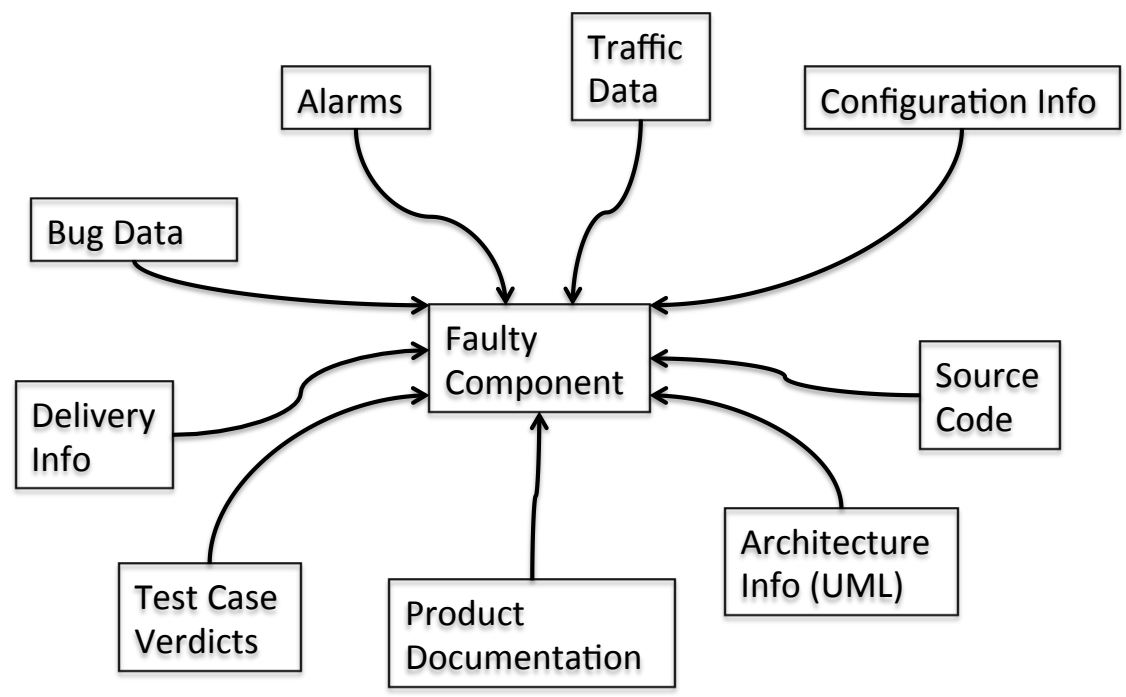

Figure 6.2: There are often many sources that, taken together, could help identify the component that is most likely contain a bug.

Other examples include test case verdicts. If a particular component has many failing test cases on a particular release, that could indicate problems with that component. 
We can also imagine including a spatial dimension in the fault localization model to exploit information from UML diagrams that describe the software architecture. With such information we can model "closeness" between components. This would mean that if one component is "suspect", the likelihood of problems with "neighboring" components would also increase. Other information from Figure 6.2 could also be included, such as configuration information, with which one could model whether a component is activated or not in the running system. If a component is not active, it probably reduces the likelihood of it being the cause of a particular bug.

Another avenue is integrating delivery information, such as which files have changed in the release on which the bug was reported. Ostrand, Weyuker, and Bell [52] have shown in a series of publications that change information is a strong indicator of which files contain bugs. By mapping files to components this approach could easily be integrated into our approach.

Integrating further contextual information into the fault localization scenario seems to us to be a fruitful future field of study.

\subsection{An End-to-end View of Automated System Analysis}

In terms of efficiency, more and more parts of the maintenance chain should be automated. Figure 6.3 visualizes this idea. Starting at the customer site, the running system should automatically detect anomalies and then automatically turn on the correct tracing. For this to be feasible, a system must be trained on historical data. The historical data must match patterns of anomalies to trace points in the system. We call these aspects Anomaly Detection and Automatic Logging.

The next step is Anomaly Reporting. When the system has concluded that a likely anomaly has occurred the system reports back to the development organization with the data that was collected by the automatic logging. This data is, of course, intended for machine analysis and is packaged in a way that allows other software to easily unpack and interpret the data. Here ABA and AFL assign the task and locate where in the system the anomaly should be fixed. At this point, Automatic Program Repair [53] kicks in, finds a solution for the fault, and automatically fixes it. Automated tests are integrated into the development flow and are continuously verifying proposed updates to the software. The new system is then automatically delivered in a Continous Delivery loop, where the old broken code is automatically replaced with new, fixed code.

This whole flow, completely automated, is not likely to be in place for many years, but this is the vision that we as a software industry should strive for. 


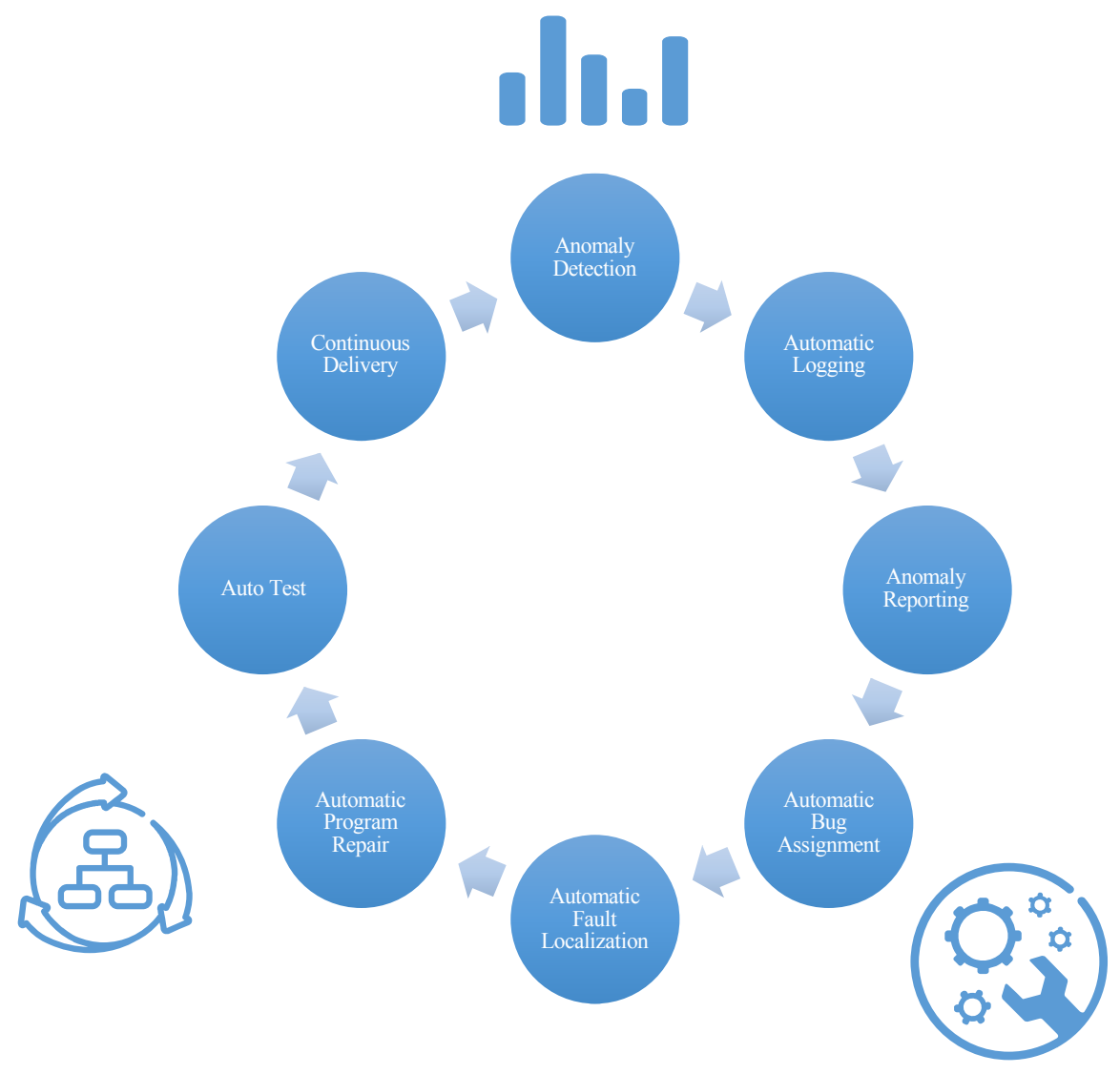

Figure 6.3: End-to-end view of automated system analysis 


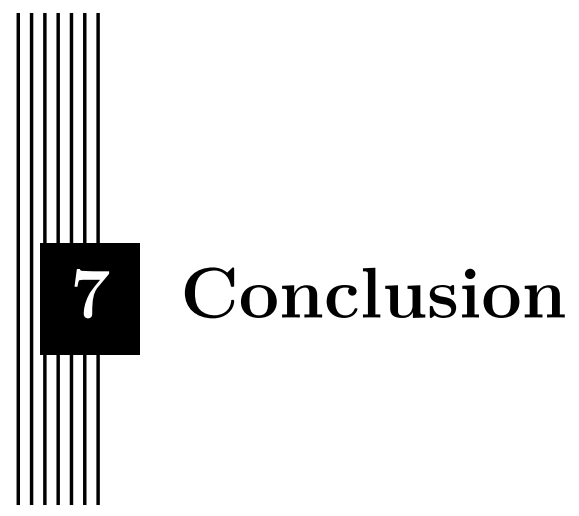

In the course of this research, we have explored the areas of Automatic Bug Assignment (ABA) and Automatic Fault Localization (AFL) in the context of large scale industrial system development. We have shown by means of studies on more than 50,000 industry bug reports that both of these techniques are feasible to deploy in an industrial context. The conclusion from papers I and II is that Stacked Generalization is a good approach when accuracy is the primary goal. It consistently outperforms individual classifiers. Based on those papers, we also conclude that if explanatory power is the focus, a richer model is needed. As part of developing such a richer model we have, in papers III and VI, derived and implemented a very fast, mathematically correct, and efficient parallel sampler for the extremely popular LDA model. This sampling approach is also applicable to other topic models. Our implementation enables researchers and companies to efficiently run LDA models on large datasets on ordinary computers without having to use and maintain high performance compute clusters. Building on our LDA sampler, we have further derived and implemented a Bayesian classifier that we call DOLDA, described in papers IV and V. DOLDA takes as input both structured data, in the form of numerical and nominal features, and unstructured text, which is represented as LDA topics. We have shown that the richness of this classifier provides many benefits in terms of both interpretability and flexibility for an organization that wants to deploy ABA or AFL in an industrial context. 



\section{Bibliography}

[1] IEEE. "IEEE Standard Classification for Software Anomalies". In: IEEE Std 1044 (2009).

[2] W Eric Wong, Ruizhi Gao, Yihao Li, Rui Abreu, and Franz Wotawa. "A survey on software fault localization". In: IEEE Transactions on Software Engineering 42.8 (2016), pp. 707-740.

[3] Kristian Wiklund. "Impediments for Automated Software Test Execution". PhD thesis. Mälardalen University, 2015.

[4] David M Blei, Andrew Y Ng, and Michael I Jordan. "Latent Dirichlet Allocation". In: Journal of Machine Learning Research 3.1 (2003), pp. 993-1022.

[5] Yguaratã Cerqueira Cavalcanti, Paulo Anselmo Mota Silveira Neto, Ivan do Carmo Machado, Tassio Ferreira Vale, Eduardo Santana Almeida, and Silvio Romero de Lemos Meira. "Challenges and opportunities for software change request repositories: a systematic mapping study". In: Journal of Software: Evolution and Process 26.7 (2014), pp. 620-653.

[6] Gregory Tassey. "The economic impacts of inadequate infrastructure for software testing". In: National Institute of Standards and Technology, RTI Project 7007.011 (2002).

[7] Alexander Terenin, Måns Magnusson, Leif Jonsson, and David Draper. "Polya Urn Latent Dirichlet Allocation: a doubly sparse massively parallel sampler". English. In: Minor revision in IEEE Transactions on Pattern Analysis and Machine Intelligence (2017). 
[8] Kalyanasundaram Somasundaram and Gail C Murphy. "Automatic categorization of bug reports using latent Dirichlet allocation". In: Proceedings of the 5th India Software Engineering Conference ISEC12. ISEC '12 (2012), pp. 125-130.

[9] J. Anvik, L. Hiew, and G. C. Murphy. "Who should fix this bug?" In: Proceedings of the 28th international conference on Software engineering. ICSE '06. Shanghai, China: ACM, 2006, pp. 361-370. ISBN: 1-59593-375-1.

[10] D. Cubranic and G. C. Murphy. "Automatic bug triage using text categorization". In: In SEKE 2004: Proceedings of the Sixteenth International Conference on Software Engineering 8 Knowledge Engineering. KSI Press, 2004, pp. 92-97.

[11] Liguo Chen, Xiaobo Wang, and Chao Liu. "An Approach to Improving Bug Assignment with Bug Tossing Graphs and Bug Similarities". In: Journal of Software 6.3 (2011). ISSN: 1796-217X.

[12] Huzefa Kagdi, Malcom Gethers, Denys Poshyvanyk, and Maen Hammad. "Assigning change requests to software developers". en. In: Journal of Software: Evolution and Process 24.1 (2012), pp. 3-33. ISSN: 20477481.

[13] N.K. Nagwani and S. Verma. "Predicting expert developers for newly reported bugs using frequent terms similarities of bug attributes". In: Proc. of the 9th International Conference on ICT and Knowledge Engineering. 2012, pp. 113-117.

[14] R. Shokripour, Z.M. Kasirun, S. Zamani, and J. Anvik. "Automatic Bug Assignment Using Information Extraction Methods". In: Proc. of the International Conference on Advanced Computer Science Applications and Technologies. 2012, pp. 144-149.

[15] H. Holmstrom, E.O. Conchuir, P.J. Agerfalk, and B. Fitzgerald. "Global Software Development Challenges: A Case Study on Temporal, Geographical and Socio-Cultural Distance". In: Global Software Engineering, 2006. ICGSE '06. International Conference on. Oct. 2006, pp. 311.

[16] Tuomas Jaanu, Maria Paasivaara, and Casper Lassenius. "Effects of Four Distances on Communication Processes in Global Software Projects". In: Proceedings of the ACM-IEEE International Symposium on Empirical Software Engineering and Measurement. ESEM '12. Lund, Sweden: ACM, 2012, pp. 231-234. ISBN: 978-1-4503-1056-7. 
[17] Elizabeth Bjarnason, Per Runeson, Markus Borg, Michael Unterkalmsteiner, Emelie Engström, Björn Regnell, Giedre Sabaliauskaite, Annabella Loconsole, Tony Gorschek, and Robert Feldt. "Challenges and practices in aligning requirements with verification and validation: a case study of six companies". English. In: Empirical Software Engineering 19.6 (2014), pp. 1809-1855. ISSN: 1382-3256.

[18] Giedre Sabaliauskaite, Annabella Loconsole, Emelie Engström, Michael Unterkalmsteiner, Björn Regnell, Per Runeson, Tony Gorschek, and Robert Feldt. "Challenges in Aligning Requirements Engineering and Verification in a Large-Scale Industrial Context". English. In: Requirements Engineering: Foundation for Software Quality. Ed. by Roel Wieringa and Anne Persson. Vol. 6182. Lecture Notes in Computer Science. Springer Berlin Heidelberg, 2010, pp. 128-142. ISBN: 978-3-64214191-1.

[19] Sigrid Eldh. "On Test Design". PhD thesis. Mälardalen University Press, Oct. 2011. URL: http: / / www.es.mdh. se/publications / 2234-.

[20] Nicolas Bettenburg, Sascha Just, Adrian Schröter, Cathrin Weiss, Rahul Premraj, and Thomas Zimmermann. "What Makes a Good Bug Report?" In: Proceedings of the 16th ACM SIGSOFT International Symposium on Foundations of Software Engineering. SIGSOFT '08/FSE-16. Atlanta, Georgia: ACM, 2008, pp. 308-318. ISBN: 978-1-59593-995-1.

[21] J. Anvik, L. Hiew, and G. C. Murphy. "Who should fix this bug?" In: Proc. of the 28th International Conference on Software Engineering. '06. New York, NY, USA, 2006, pp. 361-370. ISBN: 1-59593-375-1.

[22] Rui Abreu, Peter Zoeteweij, Rob Golsteijn, and Arjan J.C. van Gemund. "A practical evaluation of spectrum-based fault localization". In: Journal of Systems and Software 82.11 (2009), pp. 1780-1792. ISSN: 01641212.

[23] C. Wohlin, P. Runeson, M. Host, M. Ohlsson, B. Regnell, and A. Wesslen. Experimentation in Software Engineering: A Practical Guide. Springer, 2012.

[24] Per Runeson, Martin Host, Austen Rainer, and Bjorn Regnell. Case Study Research in Software Engineering: Guidelines and Examples. 1st. Wiley Publishing, 2012. ISBN: 1118104358, 9781118104354.

[25] Victor R. Basili. Software Modeling and Measurement: The Goal/Question/Metric Paradigm. Tech. rep. College Park, MD, USA, 1992. 
[26] Chris Parnin and Alessandro Orso. "Are automated debugging techniques actually helping programmers?" In: Proceedings of the 2011 International Symposium on Software Testing and Analysis ISSTA 11 (2011), p. 199.

[27] G.A. Di Lucca, M. Di Penta, and S. Gradara. "An approach to classify software maintenance requests". In: Software Maintenance, 2002. Proceedings. International Conference on. 2002, pp. 93-102.

[28] R. Rao, G. Fung, and R. Rosales. "On the Dangers of Cross-Validation. An Experimental Evaluation". In: Proc. of the SIAM International Conference on Data Mining. 2008, pp. 588-596. ISBN: 978-0-89871-654-2.

[29] C. M. Bishop. Pattern Recognition and Machine Learning. New York: Springer-Verlag, 2006. ISBN: 978-0387-31073-2.

[30] Kevin P Murphy. Machine learning: a probabilistic perspective. MIT press, 2012.

[31] Joseph K Blitzstein and Jessica Hwang. Introduction to probability. CRC Press, 2014.

[32] C. M. Bishop. Pattern Recognition and Machine Learning. New York: Springer, 2006. ISBN: 978-0387-31073-2.

[33] Tom M Mitchell. Machine learning. McGraw Hill, 1997.

[34] Leif Jonsson, Markus Borg, David Broman, Kristian Sandahl, Sigrid Eldh, and Per Runeson. "Automated bug assignment: Ensemble-based machine learning in large scale industrial contexts". English. In: Empirical Software Engineering (2016), pp. 1533-1578. ISSN: 1382-3256.

[35] Måns Magnusson, Leif Jonsson, Mattias Villani, and David Broman. "Sparse Partially Collapsed MCMC for Parallel Inference in Topic Models". In: Journal of Computational and Graphical Statistics (Aug. 2017).

[36] Andrew Gelman, John B Carlin, Hal S Stern, and Donald B Rubin. Bayesian data analysis. Vol. 2. Chapman \& Hall/CRC Boca Raton, FL, USA, 2014.

[37] Edwin T Jaynes. Probability theory: the logic of science. Cambridge university press, 2003.

[38] Laurens van der Maaten and Geoffrey Hinton. "Visualizing data using t-SNE". In: Journal of Machine Learning Research 9.Nov (2008), pp. 2579-2605.

[39] Laurens Van Der Maaten. "Accelerating t-SNE using tree-based algorithms." In: Journal of machine learning research 15.1 (2014), pp. 32213245 . 
[40] Ron Kohavi. "A Study of Cross-validation and Bootstrap for Accuracy Estimation and Model Selection". In: Proc. of the 14th International Joint Conference on Artificial Intelligence - Volume 2. 1995, pp. 11371143. ISBN: 1-55860-363-8.

[41] David Newman, Arthur Asuncion, Padhraic Smyth, and Max Welling. "Distributed algorithms for topic models". In: The Journal of Machine Learning Research 10 (2009), pp. 1801-1828.

[42] J. Yuan, F. Gao, Q. Ho, W. Dai, J. Wei, X. Zheng, E. P. Xing, T.-Y. Liu, and W.-Y. Ma. "LightLDA: Big Topic Models on Modest Compute Clusters". In: ArXiv e-prints (Dec. 2014). arXiv: 1412.1576 [stat.ML].

[43] Ian Goodfellow, Yoshua Bengio, and Aaron Courville. Deep Learning. http: //www. deeplearningbook.org. MIT Press, 2016.

[44] Carlos M. Carvalho, Nicholas G. Polson, and James G. Scott. "The horseshoe estimator for sparse signals". In: Biometrika 97.2 (2010), pp. 465-480. ISSN: 00063444.

[45] Pamela Bhattacharya, Iulian Neamtiu, and Christian R. Shelton. "Automated, highly-accurate, bug assignment using machine learning and tossing graphs". In: Journal of Systems and Software 85.10 (2012), pp. 2275-2292. ISSN: 0164-1212.

[46] Nitesh V Chawla, Nathalie Japkowicz, and Aleksander Kotcz. "Special issue on learning from imbalanced data sets". In: ACM Sigkdd Explorations Newsletter 6.1 (2004), pp. 1-6.

[47] Haibo He and Edwardo A Garcia. "Learning from imbalanced data". In: IEEE Transactions on knowledge and data engineering 21.9 (2009), pp. $1263-1284$.

[48] Haibo He and Yunqian Ma. Imbalanced learning: foundations, algorithms, and applications. John Wiley \& Sons, 2013.

[49] John Anvik. "Assisting Bug Report Triage through Recommendation". PhD thesis. University of British Columbia, 2007.

[50] Nicolò Cesa-Bianchi, Claudio Gentile, and Luca Zaniboni. "Hierarchical classification: combining Bayes with SVM". In: Proceedings of the 23rd international conference on Machine learning. ACM. 2006, pp. 177-184.

[51] Lijuan Cai and Thomas Hofmann. "Hierarchical document categorization with support vector machines". In: Proceedings of the thirteenth ACM international conference on Information and knowledge management. ACM. 2004, pp. 78-87.

[52] T. J. Ostrand, E.J. Weyuker, and R.M. Bell. "Predicting the location and number of faults in large software systems". In: IEEE Transactions on Software Engineering 31.4 (Apr. 2005), pp. 340-355. ISSN: 0098-5589. 
[53] Claire Le Goues, ThanhVu Nguyen, Stephanie Forrest, and Westley Weimer. "Genprog: A generic method for automatic software repair". In: Ieee transactions on software engineering 38.1 (2012), pp. 54-72.

[54] Leif Jonsson, David Broman, Kristian Sandahl, and Sigrid Eldh. "Towards Automated Anomaly Report Assignment in Large Complex Systems Using Stacked Generalization". In: Software Testing, Verification and Validation (ICST), 2012 IEEE Fifth International Conference on. Apr. 2012, pp. 437-446.

[55] Måns Magnusson, Leif Jonsson, Mattias Villani, and David Broman. "Sparse Partially Collapsed MCMC for Parallel Inference in Topic Models". In: Journal of Computational and Graphical Statistics (Aug. 2017).

[56] Måns Magnusson, Leif Jonsson, and Mattias Villani. "DOLDA - A Regularized Supervised Topic Model for High-dimensional Multi-class Regression". In: Revision resubmitted to Journal of Computational Statistics (June 2017).

[57] Leif Jonsson, David Broman, Måns Magnusson, Kristian Sandahl, Mattias Villani, and Sigrid Eldh. "Automatic Localization of Bugs to Faulty Components in Large Scale Software Systems using Bayesian Classification". In: Software Quality, Reliability and Security (QRS), 2016 IEEE International Conference on. IEEE. 2016, pp. 423-430. 


\section{Papers}

The papers associated with this thesis have been removed for copyright reasons. For more details about these see:

http://urn.kb.se/resolve?urn=urn:nbn:se:liu:diva-147059 


\section{Dissertations}

\section{Linköping Studies in Science and Technology Linköping Studies in Arts and Science \\ Linköping Studies in Statistics \\ Linköping Studies in Information Science}

Linköping Studies in Science and Technology

No 14 Anders Haraldsson: A Program Manipulation System Based on Partial Evaluation, 1977, ISBN 917372-144-1.

No 17 Bengt Magnhagen: Probability Based Verification of Time Margins in Digital Designs, 1977, ISBN 91-7372157-3.

No 18 Mats Cedwall: Semantisk analys av processbeskrivningar i naturligt språk, 1977, ISBN 91- 7372168-9.

No 22 Jaak Urmi: A Machine Independent LISP Compiler and its Implications for Ideal Hardware, 1978, ISBN 91-7372-188-3.

No 33 Tore Risch: Compilation of Multiple File Queries in a Meta-Database System, 1978, ISBN 91- 7372-232-4.

No 51 Erland Jungert: Synthesizing Database Structures from a User Oriented Data Model, 1980, ISBN 917372-387-8.

No 54 Sture Hägglund: Contributions to the Development of Methods and Tools for Interactive Design of Applications Software, 1980, ISBN 91-7372-404-1.

No 55 Pär Emanuelson: Performance Enhancement in a Well-Structured Pattern Matcher through Partial Evaluation, 1980, ISBN 91-7372-403-3.

No 58 Bengt Johnsson, Bertil Andersson: The HumanComputer Interface in Commercial Systems, 1981, ISBN 91-7372-414-9.

No 69 H. Jan Komorowski: A Specification of an Abstract Prolog Machine and its Application to Partial Evaluation, 1981, ISBN 91-7372-479-3.

No 71 René Reboh: Knowledge Engineering Techniques and Tools for Expert Systems, 1981, ISBN 91-7372489-0.

No 77 Östen Oskarsson: Mechanisms of Modifiability in large Software Systems, 1982, ISBN 91- 7372-527-7.

No 94 Hans Lunell: Code Generator Writing Systems, 1983, ISBN 91-7372-652-4.

No 97 Andrzej Lingas: Advances in Minimum Weight Triangulation, 1983, ISBN 91-7372-660-5.

No 109 Peter Fritzson: Towards a Distributed Programming Environment based on Incremental Compilation, 1984, ISBN 91-7372-801-2.

No 111 Erik Tengvald: The Design of Expert Planning Systems. An Experimental Operations Planning System for Turning, 1984, ISBN 91-7372- 805-5.

No 155 Christos Levcopoulos: Heuristics for Minimum Decompositions of Polygons, 1987, ISBN 91-7870133-3.

No 165 James W. Goodwin: A Theory and System for NonMonotonic Reasoning, 1987, ISBN 91-7870-183-X.

No 170 Zebo Peng: A Formal Methodology for Automated Synthesis of VLSI Systems, 1987, ISBN 91-7870-225-9.

No 174 Johan Fagerström: A Paradigm and System for Design of Distributed Systems, 1988, ISBN 91-7870301-8.

No 192 Dimiter Driankov: Towards a Many Valued Logic of Quantified Belief, 1988, ISBN 91-7870-374-3.
No 213 Lin Padgham: Non-Monotonic Inheritance for an Object Oriented Knowledge Base, 1989, ISBN 917870-485-5.

No 214 Tony Larsson: A Formal Hardware Description and Verification Method, 1989, ISBN 91-7870-517-7.

No 221 Michael Reinfrank: Fundamentals and Logical Foundations of Truth Maintenance, 1989, ISBN 917870-546-0.

No 239 Jonas Löwgren: Knowledge-Based Design Support and Discourse Management in User Interface Management Systems, 1991, ISBN 91-7870-720-X.

No 244 Henrik Eriksson: Meta-Tool Support for Knowledge Acquisition, 1991, ISBN 91-7870-746-3.

No 252 Peter Eklund: An Epistemic Approach to Interactive Design in Multiple Inheritance Hierarchies, 1991, ISBN 91-7870-784-6.

No 258 Patrick Doherty: NML3 - A Non-Monotonic Formalism with Explicit Defaults, 1991, ISBN 917870-816-8.

No 260 Nahid Shahmehri: Generalized Algorithmic Debugging, 1991, ISBN 91-7870-828-1.

No 264 Nils Dahlbäck: Representation of DiscourseCognitive and Computational Aspects, 1992, ISBN 91-7870-850-8.

No 265 Ulf Nilsson: Abstract Interpretations and Abstract Machines: Contributions to a Methodology for the Implementation of Logic Programs, 1992, ISBN 917870-858-3.

No 270 Ralph Rönnquist: Theory and Practice of Tensebound Object References, 1992, ISBN 91-7870-873-7.

No 273 Björn Fjellborg: Pipeline Extraction for VLSI Data Path Synthesis, 1992, ISBN 91-7870-880-X.

No 276 Staffan Bonnier: A Formal Basis for Horn Clause Logic with External Polymorphic Functions, 1992, ISBN 91-7870-896-6.

No 277 Kristian Sandahl: Developing Knowledge Management Systems with an Active Expert Methodology, 1992, ISBN 91-7870-897-4.

No 281 Christer Bäckström: Computational Complexity of Reasoning about Plans, 1992, ISBN 91-7870-979-2.

No 292 Mats Wirén: Studies in Incremental Natural Language Analysis, 1992, ISBN 91-7871-027-8.

No 297 Mariam Kamkar: Interprocedural Dynamic Slicing with Applications to Debugging and Testing, 1993, ISBN 91-7871-065-0.

No 302 Tingting Zhang: A Study in Diagnosis Using Classification and Defaults, 1993, ISBN 91-7871-0782.

No 312 Arne Jönsson: Dialogue Management for Natural Language Interfaces - An Empirical Approach, 1993, ISBN 91-7871-110-X

No 338 Simin Nadjm-Tehrani: Reactive Systems in Physical Environments: Compositional Modelling and Framework for Verification, 1994, ISBN 91-7871-237-8.

No 371 Bengt Savén: Business Models for Decision Support and Learning. A Study of Discrete-Event Manufacturing Simulation at Asea/ABB 1968-1993, 1995, ISBN 91-7871-494-X. 
No 375 Ulf Söderman: Conceptual Modelling of Mode Switching Physical Systems, 1995, ISBN 91-7871-5164.

No 383 Andreas Kågedal: Exploiting Groundness in Logic Programs, 1995, ISBN 91-7871-538-5.

No 396 George Fodor: Ontological Control, Description, Identification and Recovery from Problematic Control Situations, 1995, ISBN 91-7871-603-9.

No 413 Mikael Pettersson: Compiling Natural Semantics, 1995, ISBN 91-7871-641-1.

No 414 Xinli Gu: RT Level Testability Improvement by Testability Analysis and Transformations, 1996, ISBN 91-7871-654-3.

No 416 Hua Shu: Distributed Default Reasoning, 1996, ISBN 91-7871-665-9.

No 429 Jaime Villegas: Simulation Supported Industrial Training from an Organisational Learning Perspective - Development and Evaluation of the SSIT Method, 1996, ISBN 91-7871-700-0.

No 431 Peter Jonsson: Studies in Action Planning: Algorithms and Complexity, 1996, ISBN 91-7871-7043.

No 437 Johan Boye: Directional Types in Logic Programming, 1996, ISBN 91-7871-725-6.

No 439 Cecilia Sjöberg: Activities, Voices and Arenas: Participatory Design in Practice, 1996, ISBN 91-7871728-0.

No 448 Patrick Lambrix: Part-Whole Reasoning in Description Logics, 1996, ISBN 91-7871-820-1.

No 452 Kjell Orsborn: On Extensible and Object-Relational Database Technology for Finite Element Analysis Applications, 1996, ISBN 91-7871-827-9.

No 459 Olof Johansson: Development Environments for Complex Product Models, 1996, ISBN 91-7871-855-4.

No 461 Lena Strömbäck: User-Defined Constructions in Unification-Based Formalisms, 1997, ISBN 91-7871857-0.

No 462 Lars Degerstedt: Tabulation-based Logic Programming: A Multi-Level View of Query Answering, 1996, ISBN 91-7871-858-9.

No 475 Fredrik Nilsson: Strategi och ekonomisk styrning En studie av hur ekonomiska styrsystem utformas och används efter företagsförvärv, 1997, ISBN 917871-914-3.

No 480 Mikael Lindvall: An Empirical Study of Requirements-Driven Impact Analysis in Object-Oriented Software Evolution, 1997, ISBN 91-7871-927-5.

No 485 Göran Forslund: Opinion-Based Systems: The Cooperative Perspective on Knowledge-Based Decision Support, 1997, ISBN 91-7871-938-0.

No 494 Martin Sköld: Active Database Management Systems for Monitoring and Control, 1997, ISBN 917219-002-7.

No 495 Hans Olsén: Automatic Verification of Petri Nets in a CLP framework, 1997, ISBN 91-7219-011-6.

No 498 Thomas Drakengren: Algorithms and Complexity for Temporal and Spatial Formalisms, 1997, ISBN 917219-019-1.

No 502 Jakob Axelsson: Analysis and Synthesis of Heterogeneous Real-Time Systems, 1997, ISBN 917219-035-3.

No 503 Johan Ringström: Compiler Generation for DataParallel Programming Languages from Two-Level Semantics Specifications, 1997, ISBN 91-7219-045-0.

No 512 Anna Moberg: Närhet och distans - Studier av kommunikationsmönster i satellitkontor och flexibla kontor, 1997, ISBN 91-7219-119-8.
No 520 Mikael Ronström: Design and Modelling of a Parallel Data Server for Telecom Applications, 1998, ISBN 91-7219-169-4.

No 522 Niclas Ohlsson: Towards Effective Fault Prevention - An Empirical Study in Software Engineering, 1998, ISBN 91-7219-176-7.

No 526 Joachim Karlsson: A Systematic Approach for Prioritizing Software Requirements, 1998, ISBN 917219-184-8.

No 530 Henrik Nilsson: Declarative Debugging for Lazy Functional Languages, 1998, ISBN 91-7219-197-X.

No 555 Jonas Hallberg: Timing Issues in High-Level Synthesis, 1998, ISBN 91-7219-369-7.

No 561 Ling Lin: Management of 1-D Sequence Data - From Discrete to Continuous, 1999, ISBN 91-7219-402-2.

No 563 Eva L Ragnemalm: Student Modelling based on Collaborative Dialogue with a Learning Companion, 1999, ISBN 91-7219-412-X.

No 567 Jörgen Lindström: Does Distance matter? On geographical dispersion in organisations, 1999, ISBN 917219-439-1.

No 582 Vanja Josifovski: Design, Implementation and Evaluation of a Distributed Mediator System for Data Integration, 1999, ISBN 91-7219-482-0.

No 589 Rita Kovordányi: Modeling and Simulating Inhibitory Mechanisms in Mental Image Reinterpretation - Towards Cooperative HumanComputer Creativity, 1999, ISBN 91-7219-506-1.

No 592 Mikael Ericsson: Supporting the Use of Design Knowledge - An Assessment of Commenting Agents, 1999, ISBN 91-7219-532-0.

No 593 Lars Karlsson: Actions, Interactions and Narratives, 1999, ISBN 91-7219-534-7.

No 594 C. G. Mikael Johansson: Social and Organizational Aspects of Requirements Engineering Methods - A practice-oriented approach, 1999, ISBN 91-7219-541$X$.

No 595 Jörgen Hansson: Value-Driven Multi-Class Overload Management in Real-Time Database Systems, 1999, ISBN 91-7219-542-8.

No 596 Niklas Hallberg: Incorporating User Values in the Design of Information Systems and Services in the Public Sector: A Methods Approach, 1999, ISBN 917219-543-6.

No 597 Vivian Vimarlund: An Economic Perspective on the Analysis of Impacts of Information Technology: From Case Studies in Health-Care towards General Models and Theories, 1999, ISBN 91-7219-544-4.

No 598 Johan Jenvald: Methods and Tools in ComputerSupported Taskforce Training, 1999, ISBN 91-7219547-9.

No 607 Magnus Merkel: Understanding and enhancing translation by parallel text processing, 1999, ISBN 917219-614-9.

No 611 Silvia Coradeschi: Anchoring symbols to sensory data, 1999, ISBN 91-7219-623-8.

No 613 Man Lin: Analysis and Synthesis of Reactive Systems: A Generic Layered Architecture Perspective, 1999, ISBN 91-7219-630-0.

No 618 Jimmy Tjäder: Systemimplementering i praktiken En studie av logiker i fyra projekt, 1999, ISBN 917219-657-2.

No 627 Vadim Engelson: Tools for Design, Interactive Simulation, and Visualization of Object-Oriented Models in Scientific Computing, 2000, ISBN 91-7219709-9. 
No 637 Esa Falkenroth: Database Technology for Control and Simulation, 2000, ISBN 91-7219-766-8.

No 639 Per-Arne Persson: Bringing Power and Knowledge Together: Information Systems Design for Autonomy and Control in Command Work, 2000, ISBN 91-7219796-X.

No 660 Erik Larsson: An Integrated System-Level Design for Testability Methodology, 2000, ISBN 91-7219-8907.

No 688 Marcus Bjäreland: Model-based Execution Monitoring, 2001, ISBN 91-7373-016-5.

No 689 Joakim Gustafsson: Extending Temporal Action Logic, 2001, ISBN 91-7373-017-3.

No 720 Carl-Johan Petri: Organizational Information Provision - Managing Mandatory and Discretionary Use of Information Technology, 2001, ISBN 91-7373-1269.

No 724 Paul Scerri: Designing Agents for Systems with Adjustable Autonomy, 2001, ISBN 91-7373-207-9.

No 725 Tim Heyer: Semantic Inspection of Software Artifacts: From Theory to Practice, 2001, ISBN 917373-208-7.

No 726 Pär Carlshamre: A Usability Perspective on Requirements Engineering - From Methodology to Product Development, 2001, ISBN 91-7373-212-5.

No 732 Juha Takkinen: From Information Management to Task Management in Electronic Mail, 2002, ISBN 917373-258-3.

No 745 Johan Åberg: Live Help Systems: An Approach to Intelligent Help for Web Information Systems, 2002, ISBN 91-7373-311-3.

No 746 Rego Granlund: Monitoring Distributed Teamwork Training, 2002, ISBN 91-7373-312-1.

No 757 Henrik André-Jönsson: Indexing Strategies for Time Series Data, 2002, ISBN 917373-346-6.

No 747 Anneli Hagdahl: Development of IT-supported Interorganisational Collaboration - A Case Study in the Swedish Public Sector, 2002, ISBN 91-7373-314-8.

No 749 Sofie Pilemalm: Information Technology for NonProfit Organisations - Extended Participatory Design of an Information System for Trade Union Shop Stewards, 2002, ISBN 91-7373-318-0.

No 765 Stefan Holmlid: Adapting users: Towards a theory of use quality, 2002, ISBN 91-7373-397-0.

No 771 Magnus Morin: Multimedia Representations of Distributed Tactical Operations, 2002, ISBN 91-7373-4217.

No 772 Pawel Pietrzak: A Type-Based Framework for Locating Errors in Constraint Logic Programs, 2002, ISBN 91-7373-422-5.

No 758 Erik Berglund: Library Communication Among Programmers Worldwide, 2002, ISBN 91-7373-349-0.

No 774 Choong-ho Yi: Modelling Object-Oriented Dynamic Systems Using a Logic-Based Framework, 2002, ISBN 91-7373-424-1.

No 779 Mathias Broxvall: A Study in the Computational Complexity of Temporal Reasoning, 2002, ISBN 917373-440-3.

No 793 Asmus Pandikow: A Generic Principle for Enabling Interoperability of Structured and Object-Oriented Analysis and Design Tools, 2002, ISBN 91-7373-479-9.

No 785 Lars Hult: Publika Informationstjänster. En studie av den Internetbaserade encyklopedins bruksegenskaper, 2003, ISBN 91-7373-461-6.

No 800 Lars Taxén: A Framework for the Coordination of Complex Systems' Development, 2003, ISBN 917373-604-X.
No 808 Klas Gäre: Tre perspektiv på förväntningar och förändringar i samband med införande av informationssystem, 2003, ISBN 91-7373-618-X.

No 821 Mikael Kindborg: Concurrent Comics programming of social agents by children, 2003, ISBN 91-7373-651-1.

No 823 Christina Ölvingson: On Development of Information Systems with GIS Functionality in Public Health Informatics: A Requirements Engineering Approach, 2003, ISBN 91-7373-656-2.

No 828 Tobias Ritzau: Memory Efficient Hard Real-Time Garbage Collection, 2003, ISBN 91-7373-666-X.

No 833 Paul Pop: Analysis and Synthesis of Communication-Intensive Heterogeneous Real-Time Systems, 2003, ISBN 91-7373-683-X.

No 852 Johan Moe: Observing the Dynamic Behaviour of Large Distributed Systems to Improve Development and Testing - An Empirical Study in Software Engineering, 2003, ISBN 91-7373-779-8.

No 867 Erik Herzog: An Approach to Systems Engineering Tool Data Representation and Exchange, 2004, ISBN 91-7373-929-4.

No 872 Aseel Berglund: Augmenting the Remote Control: Studies in Complex Information Navigation for Digital TV, 2004, ISBN 91-7373-940-5.

No 869 Jo Skåmedal: Telecommuting's Implications on Travel and Travel Patterns, 2004, ISBN 91-7373-935-9.

No 870 Linda Askenäs: The Roles of IT - Studies of Organising when Implementing and Using Enterprise Systems, 2004, ISBN 91-7373-936-7.

No 874 Annika Flycht-Eriksson: Design and Use of Ontologies in Information-Providing Dialogue Systems, 2004, ISBN 91-7373-947-2.

No 873 Peter Bunus: Debugging Techniques for EquationBased Languages, 2004, ISBN 91-7373-941-3.

No 876 Jonas Mellin: Resource-Predictable and Efficient Monitoring of Events, 2004, ISBN 91-7373-956-1.

No 883 Magnus Bång: Computing at the Speed of Paper: Ubiquitous Computing Environments for Healthcare Professionals, 2004, ISBN 91-7373-971-5.

No 882 Robert Eklund: Disfluency in Swedish humanhuman and human-machine travel booking dialogues, 2004, ISBN 91-7373-966-9.

No 887 Anders Lindström: English and other Foreign Linguistic Elements in Spoken Swedish. Studies of Productive Processes and their Modelling using Finite-State Tools, 2004, ISBN 91-7373-981-2.

No 889 Zhiping Wang: Capacity-Constrained Productioninventory systems - Modelling and Analysis in both a traditional and an e-business context, 2004, ISBN 91-85295-08-6.

No 893 Pernilla Qvarfordt: Eyes on Multimodal Interaction, 2004, ISBN 91-85295-30-2

No 910 Magnus Kald: In the Borderland between Strategy and Management Control - Theoretical Framework and Empirical Evidence, 2004, ISBN 91-85295-82-5.

No 918 Jonas Lundberg: Shaping Electronic News: Genre Perspectives on Interaction Design, 2004, ISBN 9185297-14-3.

No 900 Mattias Arvola: Shades of use: The dynamics of interaction design for sociable use, 2004, ISBN 9185295-42-6.

No 920 Luis Alejandro Cortés: Verification and Scheduling Techniques for Real-Time Embedded Systems, 2004, ISBN 91-85297-21-6.

No 929 Diana Szentivanyi: Performance Studies of FaultTolerant Middleware, 2005, ISBN 91-85297-58-5. 
No 933 Mikael Cäker: Management Accounting as Constructing and Opposing Customer Focus: Three Case Studies on Management Accounting and Customer Relations, 2005, ISBN 91-85297-64-X.

No 937 Jonas Kvarnström: TALplanner and Other Extensions to Temporal Action Logic, 2005, ISBN 9185297-75-5.

No 938 Bourhane Kadmiry: Fuzzy Gain-Scheduled Visual Servoing for Unmanned Helicopter, 2005, ISBN 9185297-76-3.

No 945 Gert Jervan: Hybrid Built-In Self-Test and Test Generation Techniques for Digital Systems, 2005, ISBN 91-85297-97-6.

No 946 Anders Arpteg: Intelligent Semi-Structured Information Extraction, 2005, ISBN 91-85297-98-4.

No 947 Ola Angelsmark: Constructing Algorithms for Constraint Satisfaction and Related Problems - Methods and Applications, 2005, ISBN 91-85297-99-2.

No 963 Calin Curescu: Utility-based Optimisation of Resource Allocation for Wireless Networks, 2005, ISBN 91-85457-07-8.

No 972 Björn Johansson: Joint Control in Dynamic Situations, 2005, ISBN 91-85457-31-0.

No 974 Dan Lawesson: An Approach to Diagnosability Analysis for Interacting Finite State Systems, 2005, ISBN 91-85457-39-6.

No 979 Claudiu Duma: Security and Trust Mechanisms for Groups in Distributed Services, 2005, ISBN 91-8545754-X.

No 983 Sorin Manolache: Analysis and Optimisation of Real-Time Systems with Stochastic Behaviour, 2005, ISBN 91-85457-60-4.

No 986 Yuxiao Zhao: Standards-Based Application Integration for Business-to-Business Communications, 2005, ISBN 91-85457-66-3.

No 1004 Patrik Haslum: Admissible Heuristics for Automated Planning, 2006, ISBN 91-85497-28-2.

No 1005 Aleksandra Tešanovic: Developing Reusable and Reconfigurable Real-Time Software using Aspects and Components, 2006, ISBN 91-85497-29-0.

No 1008 David Dinka: Role, Identity and Work: Extending the design and development agenda, 2006, ISBN 9185497-42-8.

No 1009 Iakov Nakhimovski: Contributions to the Modeling and Simulation of Mechanical Systems with Detailed Contact Analysis, 2006, ISBN 91-85497-43-X.

No 1013 Wilhelm Dahllöf: Exact Algorithms for Exact Satisfiability Problems, 2006, ISBN 91-85523-97-6.

No 1016 Levon Saldamli: PDEModelica - A High-Level Language for Modeling with Partial Differential Equations, 2006, ISBN 91-85523-84-4.

No 1017 Daniel Karlsson: Verification of Component-based Embedded System Designs, 2006, ISBN 91-85523-79-8

No 1018 Ioan Chisalita: Communication and Networking Techniques for Traffic Safety Systems, 2006, ISBN 9185523-77-1.

No 1019 Tarja Susi: The Puzzle of Social Activity - The Significance of Tools in Cognition and Cooperation, 2006, ISBN 91-85523-71-2.

No 1021 Andrzej Bednarski: Integrated Optimal Code Generation for Digital Signal Processors, 2006, ISBN 91-85523-69-0.

No 1022 Peter Aronsson: Automatic Parallelization of Equation-Based Simulation Programs, 2006, ISBN 9185523-68-2.
No 1030 Robert Nilsson: A Mutation-based Framework for Automated Testing of Timeliness, 2006, ISBN 9185523-35-6.

No 1034 Jon Edvardsson: Techniques for Automatic Generation of Tests from Programs and Specifications, 2006, ISBN 91-85523-31-3.

No 1035 Vaida Jakoniene: Integration of Biological Data, 2006, ISBN 91-85523-28-3.

No 1045 Genevieve Gorrell: Generalized Hebbian Algorithms for Dimensionality Reduction in Natural Language Processing, 2006, ISBN 91-85643-88-2.

No 1051 Yu-Hsing Huang: Having a New Pair of Glasses Applying Systemic Accident Models on Road Safety, 2006, ISBN 91-85643-64-5.

No 1054 Åsa Hedenskog: Perceive those things which cannot be seen - A Cognitive Systems Engineering perspective on requirements management, 2006, ISBN 91-85643-57-2.

No 1061 Cécile Åberg: An Evaluation Platform for Semantic Web Technology, 2007, ISBN 91-85643-31-9.

No 1073 Mats Grindal: Handling Combinatorial Explosion in Software Testing, 2007, ISBN 978-91-85715-74-9.

No 1075 Almut Herzog: Usable Security Policies for Runtime Environments, 2007, ISBN 978-91-85715-65-7.

No 1079 Magnus Wahlström: Algorithms, measures, and upper bounds for Satisfiability and related problems, 2007, ISBN 978-91-85715-55-8.

No 1083 Jesper Andersson: Dynamic Software Architectures, 2007, ISBN 978-91-85715-46-6.

No 1086 Ulf Johansson: Obtaining Accurate and Comprehensible Data Mining Models - An Evolutionary Approach, 2007, ISBN 978-91-85715-34-3.

No 1089 Traian Pop: Analysis and Optimisation of Distributed Embedded Systems with Heterogeneous Scheduling Policies, 2007, ISBN 978-91-85715-27-5.

No 1091 Gustav Nordh: Complexity Dichotomies for CSPrelated Problems, 2007, ISBN 978-91-85715-20-6.

No 1106 Per Ola Kristensson: Discrete and Continuous Shape Writing for Text Entry and Control, 2007, ISBN 978-91-85831-77-7.

No 1110 He Tan: Aligning Biomedical Ontologies, 2007, ISBN 978-91-85831-56-2.

No 1112 Jessica Lindblom: Minding the body - Interacting socially through embodied action, 2007, ISBN 978-9185831-48-7.

No 1113 Pontus Wärnestål: Dialogue Behavior Management in Conversational Recommender Systems, 2007, ISBN 978-91-85831-47-0

No 1120 Thomas Gustafsson: Management of Real-Time Data Consistency and Transient Overloads in Embedded Systems, 2007, ISBN 978-91-85831-33-3.

No 1127 Alexandru Andrei: Energy Efficient and Predictable Design of Real-time Embedded Systems, 2007, ISBN 978-91-85831-06-7.

No 1139 Per Wikberg: Eliciting Knowledge from Experts in Modeling of Complex Systems: Managing Variation and Interactions, 2007, ISBN 978-91-85895-66-3.

No 1143 Mehdi Amirijoo: QoS Control of Real-Time Data Services under Uncertain Workload, 2007, ISBN 97891-85895-49-6.

No 1150 Sanny Syberfeldt: Optimistic Replication with Forward Conflict Resolution in Distributed Real-Time Databases, 2007, ISBN 978-91-85895-27-4.

No 1155 Beatrice Alenljung: Envisioning a Future Decision Support System for Requirements Engineering - A Holistic and Human-centred Perspective, 2008, ISBN 978-91-85895-11-3. 
No 1156 Artur Wilk: Types for XML with Application to Xcerpt, 2008, ISBN 978-91-85895-08-3.

No 1183 Adrian Pop: Integrated Model-Driven Development Environments for Equation-Based Object-Oriented Languages, 2008, ISBN 978-91-7393-895-2.

No 1185 Jörgen Skågeby: Gifting Technologies Ethnographic Studies of End-users and Social Media Sharing, 2008, ISBN 978-91-7393-892-1.

No 1187 Imad-Eldin Ali Abugessaisa: Analytical tools and information-sharing methods supporting road safety organizations, 2008, ISBN 978-91-7393-887-7.

No 1204 H. Joe Steinhauer: A Representation Scheme for Description and Reconstruction of Object Configurations Based on Qualitative Relations, 2008, ISBN 978-91-7393-823-5.

No 1222 Anders Larsson: Test Optimization for Core-based System-on-Chip, 2008, ISBN 978-91-7393-768-9.

No 1238 Andreas Borg: Processes and Models for Capacity Requirements in Telecommunication Systems, 2009, ISBN 978-91-7393-700-9.

No 1240 Fredrik Heintz: DyKnow: A Stream-Based Knowledge Processing Middleware Framework, 2009, ISBN 978-91-7393-696-5.

No 1241 Birgitta Lindström: Testability of Dynamic RealTime Systems, 2009, ISBN 978-91-7393-695-8.

No 1244 Eva Blomqvist: Semi-automatic Ontology Construction based on Patterns, 2009, ISBN 978-91-7393-683-5.

No 1249 Rogier Woltjer: Functional Modeling of Constraint Management in Aviation Safety and Command and Control, 2009, ISBN 978-91-7393-659-0.

No 1260 Gianpaolo Conte: Vision-Based Localization and Guidance for Unmanned Aerial Vehicles, 2009, ISBN 978-91-7393-603-3.

No 1262 AnnMarie Ericsson: Enabling Tool Support for Formal Analysis of ECA Rules, 2009, ISBN 978-91-7393598-2.

No 1266 Jiri Trnka: Exploring Tactical Command and Control: A Role-Playing Simulation Approach, 2009, ISBN 978-91-7393-571-5.

No 1268 Bahlol Rahimi: Supporting Collaborative Work through ICT - How End-users Think of and Adopt Integrated Health Information Systems, 2009, ISBN 978-91-7393-550-0.

No 1274 Fredrik Kuivinen: Algorithms and Hardness Results for Some Valued CSPs, 2009, ISBN 978-91-7393-525-8.

No 1281 Gunnar Mathiason: Virtual Full Replication for Scalable Distributed Real-Time Databases, 2009, ISBN 978-91-7393-503-6.

No 1290 Viacheslav Izosimov: Scheduling and Optimization of Fault-Tolerant Distributed Embedded Systems, 2009, ISBN 978-91-7393-482-4.

No 1294 Johan Thapper: Aspects of a Constraint Optimisation Problem, 2010, ISBN 978-91-7393-464-0.

No 1306 Susanna Nilsson: Augmentation in the Wild: User Centered Development and Evaluation of Augmented Reality Applications, 2010, ISBN 978-917393-416-9.

No 1313 Christer Thörn: On the Quality of Feature Models, 2010, ISBN 978-91-7393-394-0.

No 1321 Zhiyuan He: Temperature Aware and DefectProbability Driven Test Scheduling for System-onChip, 2010, ISBN 978-91-7393-378-0.

No 1333 David Broman: Meta-Languages and Semantics for Equation-Based Modeling and Simulation, 2010, ISBN 978-91-7393-335-3.

No 1337 Alexander Siemers: Contributions to Modelling and Visualisation of Multibody Systems Simulations with
Detailed Contact Analysis, 2010, ISBN 978-91-7393317-9.

No 1354 Mikael Asplund: Disconnected Discoveries: Availability Studies in Partitioned Networks, 2010, ISBN 978-91-7393-278-3.

No 1359 Jana Rambusch: Mind Games Extended: Understanding Gameplay as Situated Activity, 2010, ISBN 978-91-7393-252-3.

No 1373 Sonia Sangari: Head Movement Correlates to Focus Assignment in Swedish, 2011, ISBN 978-91-7393-1540 .

No 1374 Jan-Erik Källhammer: Using False Alarms when Developing Automotive Active Safety Systems, 2011, ISBN 978-91-7393-153-3.

No 1375 Mattias Eriksson: Integrated Code Generation, 2011, ISBN 978-91-7393-147-2

No 1381 Ola Leifler: Affordances and Constraints of Intelligent Decision Support for Military Command and Control - Three Case Studies of Support Systems, 2011, ISBN 978-91-7393-133-5.

No 1386 Soheil Samii: Quality-Driven Synthesis and Optimization of Embedded Control Systems, 2011, ISBN 978-91-7393-102-1.

No 1419 Erik Kuiper: Geographic Routing in Intermittentlyconnected Mobile Ad Hoc Networks: Algorithms and Performance Models, 2012, ISBN 978-91-7519981-8.

No 1451 Sara Stymne: Text Harmonization Strategies for Phrase-Based Statistical Machine Translation, 2012, ISBN 978-91-7519-887-3.

No 1455 Alberto Montebelli: Modeling the Role of Energy Management in Embodied Cognition, 2012, ISBN 978-91-7519-882-8.

No 1465 Mohammad Saifullah: Biologically-Based Interactive Neural Network Models for Visual Attention and Object Recognition, 2012, ISBN 978-91-7519-838-5.

No 1490 Tomas Bengtsson: Testing and Logic Optimization Techniques for Systems on Chip, 2012, ISBN 978-917519-742-5.

No 1481 David Byers: Improving Software Security by Preventing Known Vulnerabilities, 2012, ISBN 97891-7519-784-5.

No 1496 Tommy Färnqvist: Exploiting Structure in CSPrelated Problems, 2013, ISBN 978-91-7519-711-1.

No 1503 John Wilander: Contributions to Specification, Implementation, and Execution of Secure Software, 2013, ISBN 978-91-7519-681-7.

No 1506 Magnus Ingmarsson: Creating and Enabling the Useful Service Discovery Experience, 2013, ISBN 97891-7519-662-6.

No 1547 Wladimir Schamai: Model-Based Verification of Dynamic System Behavior against Requirements: Method, Language, and Tool, 2013, ISBN 978-917519-505-6.

No 1551 Henrik Svensson: Simulations, 2013, ISBN 978-917519-491-2.

No 1559 Sergiu Rafiliu: Stability of Adaptive Distributed Real-Time Systems with Dynamic Resource Management, 2013, ISBN 978-91-7519-471-4.

No 1581 Usman Dastgeer: Performance-aware Component Composition for GPU-based Systems, 2014, ISBN 978-91-7519-383-0.

No 1602 Cai Li: Reinforcement Learning of Locomotion based on Central Pattern Generators, 2014, ISBN 978-917519-313-7.

No 1652 Roland Samlaus: An Integrated Development Environment with Enhanced Domain-Specific 
Interactive Model Validation, 2015, ISBN 978-917519-090-7.

No 1663 Hannes Uppman: On Some Combinatorial Optimization Problems: Algorithms and Complexity, 2015, ISBN 978-91-7519-072-3.

No 1664 Martin Sjölund: Tools and Methods for Analysis, Debugging, and Performance Improvement of Equation-Based Models, 2015, ISBN 978-91-7519-071-6.

No 1666 Kristian Stavåker: Contributions to Simulation of Modelica Models on Data-Parallel Multi-Core Architectures, 2015, ISBN 978-91-7519-068-6.

No 1680 Adrian Lifa: Hardware/Software Codesign of Embedded Systems with Reconfigurable and Heterogeneous Platforms, 2015, ISBN 978-91-7519-0402.

No 1685 Bogdan Tanasa: Timing Analysis of Distributed Embedded Systems with Stochastic Workload and Reliability Constraints, 2015, ISBN 978-91-7519-022-8.

No 1691 Håkan Warnquist: Troubleshooting Trucks Automated Planning and Diagnosis, 2015, ISBN 97891-7685-993-3.

No 1702 Nima Aghaee: Thermal Issues in Testing of Advanced Systems on Chip, 2015, ISBN 978-91-7685949-0.

No 1715 Maria Vasilevskaya: Security in Embedded Systems: A Model-Based Approach with Risk Metrics, 2015, ISBN 978-91-7685-917-9.

No 1729 Ke Jiang: Security-Driven Design of Real-Time Embedded System, 2016, ISBN 978-91-7685-884-4.

No 1733 Victor Lagerkvist: Strong Partial Clones and the Complexity of Constraint Satisfaction Problems: Limitations and Applications, 2016, ISBN 978-91-7685856-1.

No 1734 Chandan Roy: An Informed System Development Approach to Tropical Cyclone Track and Intensity Forecasting, 2016, ISBN 978-91-7685-854-7.

No 1746 Amir Aminifar: Analysis, Design, and Optimization of Embedded Control Systems, 2016, ISBN 978-917685-826-4.

No 1747 Ekhiotz Vergara: Energy Modelling and Fairness for Efficient Mobile Communication, 2016, ISBN 978-91-7685-822-6.

No 1748 Dag Sonntag: Chain Graphs - Interpretations, Expressiveness and Learning Algorithms, 2016, ISBN 978-91-7685-818-9.

No 1768 Anna Vapen: Web Authentication using ThirdParties in Untrusted Environments, 2016, ISBN 978-91-7685-753-3.

No 1778 Magnus Jandinger: On a Need to Know Basis: A Conceptual and Methodological Framework for Modelling and Analysis of Information Demand in an Enterprise Context, 2016, ISBN 978-91-7685-713-7.

No 1798 Rahul Hiran: Collaborative Network Security: Targeting Wide-area Routing and Edgenetwork Attacks, 2016, ISBN 978-91-7685-662-8.

No 1813 Nicolas Melot: Algorithms and Framework for Energy Efficient Parallel Stream Computing on Many-Core Architectures, 2016, ISBN 978-91-7685623-9.

No 1823 Amy Rankin: Making Sense of Adaptations: Resilience in High-Risk Work, 2017, ISBN 978-917685-596-6.
No 1831 Lisa Malmberg: Building Design Capability in the Public Sector: Expanding the Horizons of Development, 2017, ISBN 978-91-7685-585-0.

No 1851 Marcus Bendtsen: Gated Bayesian Networks, 2017, ISBN 978-91-7685-525-6.

No 1852 Zlatan Dragisic: Completion of Ontologies and Ontology Networks, 2017, ISBN 978-91-7685-522-5.

No 1854 Meysam Aghighi: Computational Complexity of some Optimization Problems in Planning, 2017, ISBN 978-91-7685-519-5.

No 1863 Simon Ståhlberg: Methods for Detecting Unsolvable Planning Instances using Variable Projection, 2017, ISBN 978-91-7685-498-3.

No 1879 Karl Hammar: Content Ontology Design Patterns: Qualities, Methods, and Tools, 2017, ISBN 978-91-7685-454-9.

No 1887 Ivan Ukhov: System-Level Analysis and Design under Uncertainty, 2017, ISBN 978-91-7685-426-6.

No 1891 Valentina Ivanova: Fostering User Involvement in Ontology Alignment and Alignment Evaluation, 2017, ISBN 978-91-7685-403-7.

No 1902 Vengatanathan Krishnamoorthi: Efficient HTTPbased Adaptive Streaming of Linear and Interactive Videos, 2018, ISBN 978-91-7685-371-9.

No 1903 Lu Li: Programming Abstractions and Optimization Techniques for GPU-based Heterogeneous Systems, 2018, ISBN 978-91-7685370-2.

No 1913 Jonas Rybing: Studying Simulations with Distributed Cognition, 2018, ISBN 978-91-7685-3481.

No 1936 Leif Jonsson: Machine Learning-Based Bug Handling in Large-Scale Software Development, 2018, ISBN 978-91-7685-306-1.

Linköping Studies in Arts and Science

No 504 Ing-Marie Jonsson: Social and Emotional Characteristics of Speech-based In-Vehicle Information Systems: Impact on Attitude and Driving Behaviour, 2009, ISBN 978-91-7393-478-7.

No 586 Fabian Segelström: Stakeholder Engagement for Service Design: How service designers identify and communicate insights, 2013, ISBN 978-91-7519-554-4.

No 618 Johan Blomkvist: Representing Future Situations of Service: Prototyping in Service Design, 2014, ISBN 978-91-7519-343-4.

No 620 Marcus Mast: Human-Robot Interaction for SemiAutonomous Assistive Robots, 2014, ISBN 978-917519-319-9.

No 677 Peter Berggren: Assessing Shared Strategic Understanding, 2016, ISBN 978-91-7685-786-1.

No 695 Mattias Forsblad: Distributed cognition in home environments: The prospective memory and cognitive practices of older adults, 2016, ISBN 97891-7685-686-4.

\section{Linköping Studies in Statistics}

No 9 Davood Shahsavani: Computer Experiments Designed to Explore and Approximate Complex Deterministic Models, 2008, ISBN 978-91-7393-976-8.

No 10 Karl Wahlin: Roadmap for Trend Detection and Assessment of Data Quality, 2008, ISBN 978-91-7393792-4. 
No 11 Oleg Sysoev: Monotonic regression for large multivariate datasets, 2010, ISBN 978-91-7393-412-1.

No 13 Agné Burauskaite-Harju: Characterizing Temporal Change and Inter-Site Correlations in Daily and Subdaily Precipitation Extremes, 2011, ISBN 978-91-7393110-6.

No 14 Måns Magnusson: Scalable and Efficient Probabilistic Topic Model Inference for Textual Data, 2018, ISBN 978-91-7685-288-0.

\section{Linköping Studies in Information Science}

No 1 Karin Axelsson: Metodisk systemstrukturering- att skapa samstämmighet mellan informationssystemarkitektur och verksamhet, 1998. ISBN 9172-19-296-8.

No 2 Stefan Cronholm: Metodverktyg och användbarhet - en studie av datorstödd metodbaserad systemutveckling, 1998, ISBN 9172-19-299-2.

No 3 Anders Avdic: Användare och utvecklare - om anveckling med kalkylprogram, 1999. ISBN 91-7219606-8.

No 4 Owen Eriksson: Kommunikationskvalitet hos informationssystem och affärsprocesser, 2000, ISBN 917219-811-7.

No 5 Mikael Lind: Från system till process - kriterier för processbestämning vid verksamhetsanalys, 2001, ISBN 91-7373-067-X.

No 6 Ulf Melin: Koordination och informationssystem i företag och nätverk, 2002, ISBN 91-7373-278-8.

No 7 Pär J. Ågerfalk: Information Systems Actability Understanding Information Technology as a Tool for Business Action and Communication, 2003, ISBN 917373-628-7.

No 8 Ulf Seigerroth: Att förstå och förändra systemutvecklingsverksamheter - en taxonomi för metautveckling, 2003, ISBN 91-7373-736-4.

No 9 Karin Hedström: Spår av datoriseringens värden Effekter av IT i äldreomsorg, 2004, ISBN 91-7373-9634.

No 10 Ewa Braf: Knowledge Demanded for Action Studies on Knowledge Mediation in Organisations, 2004, ISBN 91-85295-47-7.

No 11 Fredrik Karlsson: Method Configuration method and computerized tool support, 2005, ISBN 91-8529748-8.

No 12 Malin Nordström: Styrbar systemförvaltning - Att organisera systemförvaltningsverksamhet med hjälp av effektiva förvaltningsobjekt, 2005, ISBN 91-85297$60-7$.

No 13 Stefan Holgersson: Yrke: POLIS - Yrkeskunskap, motivation, IT-system och andra förutsättningar för polisarbete, 2005, ISBN 91-85299-43-X.

No 14 Benneth Christiansson, Marie-Therese Christiansson: Mötet mellan process och komponent - mot ett ramverk för en verksamhetsnära kravspecifikation vid anskaffning av komponentbaserade informationssystem, 2006, ISBN 91-85643$22-X$. 